MITCH

\title{
SILICA RECOVERY AND CONTROL IN HAWAIIAN
}

GEOTHERMAL FLUIDS

\author{
Final Report \\ U.S. DOE Grant No. \\ DE-FG07-88ID12741
}

\begin{abstract}
Submitted By:
Donald M. Thomas

Hawaii Institute of Geophysics

School of Ocean and Earth Science and Technology University of Hawaii
\end{abstract}

June, 1992 


\section{Disclaimer}

This report was prepared as an account of work sponsored in part by the U.S. Department of Energy (DOE) Grant No. DE-FG07-88ID12741. However, any findings, conclusions, or recommendations expressed herein are those of the author(s) and do not necessarily reflect the views of DOE. Reference herein to any specified commercial product, process, or service by trade name, trademark, manufacturer, or otherwise does not necessarily constitute or imply its endorsement, recommendation, or favoring by the United states Government or any agency thereof. 


\begin{abstract}
A series of experiments was performed to investigate methods of controlling silica in waste geothermal brines produced at the HGP-A Generator Facility. Laboratory testing has shown that the rate of polymerization of silica in the geothermal fluids is highly $\mathrm{pH}$ dependent and increases rapidly as the brine $\mathrm{pH}$ is increased. At brine $\mathrm{pH}$ values in excess of 8.5 the suspension of silica polymers flocculated and rapidly precipitated a gelatinous silica mass. Optimum flocculation and precipitation rates were achieved at $\mathrm{pH}$ values in the range of 10.5 to 11.5 . The addition of transition metal salts to the geothermal fluids similarly increased the rate of polymerization as well as the degree of precipitation of the silica polymer from suspension. A series of experiments performed on the recovered silica solids demonstrated that methanol extraction of the water in the gels followed by critical point drying yielded surface areas in excess of $300 \mathrm{~m}^{2} / \mathrm{g}$ and that treatment of the dried solids with $2 \mathrm{~N} \mathrm{HCl}$ removed most of the adsorbed impurities in the recovered product. A series of experiments tested the response of the waste brines to mixing with steam condensate and non-condensable gases. The results demonstrated that the addition of condensate and NCG greatly increased the stability of the silica in the geothermal brines. They also indicated that the process could reduce the potential for plugging of reinjection wells receiving waste geothermal fluids from commercial geothermal facilities in Hawaii. conceptual designs were proposed to apply the gas re-combination approach to the disposal of geothermal waste fluids having a range of chemical compositions. Finally, these designs were applied to the geothermal fluid compositions found at cerro Prieto, Ahuachapan, and salton sea. our analysis indicates that they could, under appropriate circumstances, be used at Cerro Prieto and Ahuachapan but not at the salton sea geothermal field.
\end{abstract}




\section{Executive Summary}

The geothermal fluids produced by the geothermal system on the Kilauea East Rift zone typically have been found to have high concentrations of dissolved silica that may present a significant obstacle to commercial development of this high-temperature resource. In an effort to identify a process capable of controlling deposition of silica from the geothermal fluids produced by the resource, a series of experiments were performed on the waste fluids produced by the HGP-A Generator Facility. The objectives of these investigations were to: 1) Develop methods of extracting the silica from the geothermal fluids; 2) Optimize the rates of recovery as well as the physical characteristics and chemical purity of the recovered solids; 3) Identify a means of inhibiting silica deposition that will allow reinjection of the geothermal fluids; 4) Generate a conceptual design for a brine disposal system; and 5) Evaluate the feasibility of the conceptual design for use in geothermal fields in North America in which commercial geothermal development is currently underway.

The first series of experiments evaluated silica polymerization characteristics in the geothermal fluids as a function of $\mathrm{ph}$. It showed that increasing the brine $\mathrm{pH}$ from 7 to 8 resulted in a significant increase in the rate of polymerization of the silicic acid monomer and that further increases in $\mathrm{pH}$ destabilized the colloidal suspension and produced rapid agglomeration of a gelatinous floc.

The precipitation of silica from solution was found to be most rapid, and the silica floc to occupy the least volume, at a $\mathrm{pH}$ range of 10.5 to 11 . An analysis of the zeta potential of the precipitated silica flocs found that the solids carried a strong negative surface charge under slightly alkaline conditions. At pH levels of about 10.5 to 11 , however, there was a rapid weakening, and ultimately a reversal of the surface charge to positive values above pH 11. We attribute this effect to ion exchange between the surface silanol groups and multiply charged cations (e.g. Ca) present in the geothermal brines. 
The addition of low concentrations of iron or aluminum salts was also found to enhance the recovery of silica at lower pH values than with caustic alone. The effectiveness of these transition metal ions at promoting silica agglomeration is attributed to their higher charge density as well as their ability to act as bridging ions between silica polymers.

A survey of the market prices for silica established that the most valuable form of silica was as a finely divided, or high surface, area powder. The silica floc were, therefore, treated to a variety of post recovery processing conditions in an effort to optimize surface areas of the final product. The most successful treatment method developed was one in which water was extracted from the recovered gel with methanol and then dried in a supercritical $\mathrm{CO}_{2}$ drying system. Surface areas in excess of $300 \mathrm{~m}^{2} / \mathrm{g}$ were obtained with this process.

Purification of the recovered solids was tested using dilute $\mathrm{HCl}$, which was found to remove most of the adsorbed contaminants at acid concentrations of $2 \mathrm{M}$ or less.

We investigated the inhibition of silica precipitation using steam condensate and the non-condensable gases (NCG) produced by the HGP-A Generator Facility as weakly acid buffering agents for the brine. This study showed that recombination of condensate with the brine could substantially increase the stability of silica in the waste fluids. Addition of NCG further improved the stability of silica but also showed some tendency to precipitate sulfide reactive metals from the geothermal brines. Although the amounts of metal sulfides formed in the mixed phase test was indicated to be small, an absolute measurement of the precipitation rate was not possible due to air entrainment in the gases and the formation of elemental sulfur within the test system.

A conceptual design for disposal of geothermal waste fluids recommends that the saturation pressure of the brine be reduced immediately after discharge from the plant separator by the injection of steam condensate. Small amounts of NCG could be 
injected as needed to reduce the $\mathrm{pH}$ of the brines below about 5 , with the balance of the gases injected at the reinjection wellhead or below the water level in the reinjection well. If high metals concentrations are present in the geothermal brines, it is recommended that adequate gas be injected to precipitate most of the sulfide- (or carbonate-) reactive metals and that the metal sulfides be removed from the waste stream prior to reinjection into the disposal well.

An analysis of the fluid compositions found at cerro Prieto geothermal field suggests that injection of NCG into the waste fluids could successfully inhibit silica deposition but oniy if the mixed brine and gas stream is maintained under pressure throughout the reinjection process. Although the data available for Ahuachapan is more limited, it is possible that this approach could be used in this field as well. An analysis of this method for use at the salton sea geothermal field indicates that injection of NCG into the metal-rich brines produced by this field would probably generate rapid deposition of metal sulfides and metal carbonates and, hence, would not appreciably contribute to the control of solids deposition from these fluids. 


\section{Acknowledgements}

Many individuals and organizations contributed to the success of the work described here. The U.S. Department of Energy and the State of Hawaii Department of Business and Economic Development provided funding; Hawaii Electric Light Company and Puna Geothermal Venture provided technical assistance and access to the geothermal fluids and design data; Bob Taylor, Eric Decarlo, and Gary Delanoy, conducted fluid analyses and contributed geochemical and scientific support; Bob Mitiguy and Robert Kochy provided technical and field assistance; and Barbara Jones reviewed and edited the current manuscript. The assistance and cooperation of these individuals, as well as all others who contributed to the successful completion of this research effort, is gratefully acknowledged. 
TABLE OF CONTENTS

ABSTRACT . . . . . . . . . . . . . . . . . iii

EXECUTIVE SUMMARY . . . . . . . . . . . . . . iv

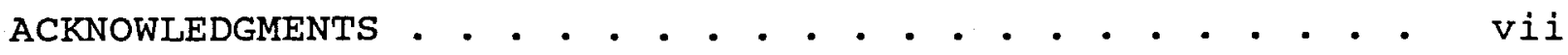

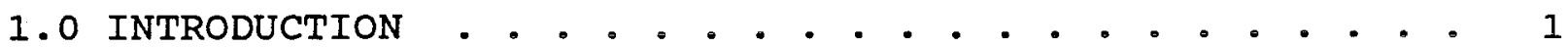

1.1 General statement . . . . . . . . . . . . . 1

1.2 Background . . . . . . . . . . . . . . . . . . 1

1.3 Experimental objectives . . . . . . . . . . . 5

2.0 SILICA PRECIPITATION STUDIES . . . . . . . . . . . . 7

2.1 Polymerization . . . . . . . . . . . . . . . 7

2.1.1 Experimental Procedure . . . . . . . . 7

2.1 .2 Results . . . . . . . . . . . . . . 7

2.1 .3 Analysis . . . . . . . . . . . . . 9

2.2 Precipitation . . . . . . . . . . . . . . . . . 12

2.2.1 Experimental Procedure . . . . . . . . 12

2.2 .2 Results . . . . . . . . . . . . . . . . . 13

2.2.3 Analysis . . . . . . . . . . . . . . 17

2.3 Batch Recovery of Silica . . . . . . . . . . 20

2.3.1 Experimental Procedure . . . . . . . . . 21

2.3.2 Results and Analysis . . . . . . . . . . 21

2.4 Summary and Conclusions . . . . . . . . . . . 26

2.5 Applications . . . . . . . . . . . . . . . 26

3.0 SILICA RECOVERY INVESTIGATIONS . . . . . . . . . 28

3.1 Background . . . . . . . . . . . . . . . . 28

3.2 Optimization of Surface Area . . . . . . . . . . 28

3.2.1 Experimental Procedure . . . . . . . . . . 29

3.2.2 Initial Results . . . . . . . . . . . 29

3.2.3 Additional Testing . . . . . . . . . 30

3.2 .4 Results... . . . . . . . . . . . . . . . 31

3.2 .5 Analysis . . . . . . . . . . . . . . 32

3.3 Contaminant Concentration and Removal . . . . . 33

3.3.1 Experimental Procedure ......... . . 33

3.3 .2 Results . . . . . . . . . . . . . . . . . . 33

3.3.3 Analysis . . . . . . . . . . . . . . 34

3.3.4 Leaching Experiments . . . . . . . . . . 39

3.4 Summary and conclusions . . . . . . . . . . . 43

4.0 SILICA INHIBITION STUDIES . . . . . . . . . . . 45

4.1 Objectives . . . . . . . . . . . . . . . . . . . 45

4.2 Test Stand Design ................ 45

4.3 Non-Condensable Gas Injection Test Plan . . . . 49

4.4 Experimental Trials Performed . . . . . . . . . . 52

4.4.1 Brine/Condensate Mixing . . . . . . . . . 52

4.4.2 Brine/Condensate/Non-Condensable Gas Mixing 53 
4.5 Brine/Condensate Trial . . . . . . . . . . 54 4.5.1 Homogeneous Precipitation. . . . . . . . . 54 4.5.2 Corrosion and Heterogeneous Precipitation . 55

4.6 Brine/Condensate/Non-Condensable Gas Trial . . . 62 4.6.1 Gas Mixing and $\mathrm{pH}$. . . . . . . . . . . 62 4.6.2 Homogeneous Precipitation . . . . . . . . 63 4.6.3 Corrosion and Heterogeneous Precipitation . 63

4.7 Conclusions and Recommendations . . . . . . . 65

5.0 CONCEPTUAL DESIGN OF A SILICA CONTROL PROCESS . . . . . 66

5.1 Process Design . . . . . . . . . . . . . . . . . 66

5.2 Low Metals Geothermal Fluids . . . . . . . . . 66

5.3 High Metals Geothermal Fluids . . . . . . . . 69

5.4 Summary . . . . . . . . . . . . . . . . . . 71

6. 0 APPLICATION OF SILICA CONTROL TO NORTH AMERICAN

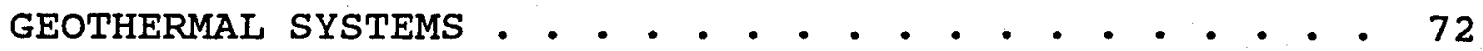

6.1 Cerro Prieto . . . . . . . . . . . . . . . 72

6.2 Ahuachapan . . . . . . . . . . . . . . . . 73

6.3 Salton Sea . . . . . . . . . . . . . . . . 74

6.4 Summary and Conclusions . . . . . . . . . . 75

7.0 BIBLIOGRAPHY . . . . . . . . . . . . . . . . . . 76

8.0 APPENDIX I • • . . . . . . . . . . . . . . . . . . 82

8.1 Statement of Work . . . . . . . . . . . . . 82

9.0 APPENDIX II . . . . . . . . . . . . . . . . . . . 84

9.1 Experimental Protocol for Gas Mixing System . . . 84 


\section{LIST OF FIGURES}

1. Location of the HGP-A Geothermal Generator Facility

and Kilauea East Rift Zone hydrothermal system. . . . 3

2. Line diagram of HGP-A Geothermal Generator Facility

process flow. . . . . . . . . . . . . . . . 4

3. Decline in molybdate reactive silica concentrations with time. . . . . . . . . . . . . . . . 8

4. Analysis of silica decline curves following Flemming's (1986) suggested procedure. . . . . . . 10

5. Residual total silica present in geothermal brines as a function of $\mathrm{pH}$ of treatment. . . . . . . . . . . 14

6. Rate of settling of agglomerated silica floc as a function of $\mathrm{pH}$ : intermediate $\mathrm{pH}$ treatments. . . . 15

7. Rate of settling of agglomerated silica floc as a function of $\mathrm{pH}$ : high $\mathrm{pH}$ treatments. . . . . . . 16

8. Zeta potential of recovered silica floc as a function of $\mathrm{pH}$ of precipitation. . . . . . . . . 18

9. Residual total silica present in geothermal fluid as a function of iron and aluminum treatments. . . . . 22

10. Residual total silica present in geothermal fluid as a function of iron addition for flashed and unflashed brines. . . 24

11. Residual total silica present in geothermal fluid as a function of aluminum addition for flashed and unflashed brines . 25

12. Alkali metals enrichment in silica precipitate from HGP-A geothermal fluids . . . . . . . . 36

13. Trace transition metals enrichment in silica precipitate from HGP-A geothermal fluids . . . . . . 38

14. Acid leaching of alkali and transition metals from silica precipitated from geothermal fluid by iron addition . . 40

15. Acid leaching of alkali and transition metals from silica precipitated from geothermal fluid by aluminum sulfate addition 
16. Acid leaching of alkali and transition metals from silica precipitated from geothermal fluid by potassium aluminum sulfate addition . . . . . . . . . . . .

17. Line diagram of the bench-scale test system fabricated for mixing geothermal brine with silica precipitating and silica inhibiting reagents 


\section{LIST OF PLATES}

1. Mixed geothermal brine and steam condensate showing absence of suspended particulates immediately after mixing and filtration . . . . . . . . . . . . 56

2. Mixed geothermal brine and steam condensate, as in Plate 1 , after 24 hours of polymerization at ambient temperature 56

3. Pure geothermal brine immediately after flashing to atmospheric pressure and $100^{\circ} \mathrm{C}$ showing high concentration of colloidal suspended particulates . . . . . . . . 57

4. Scanning electron micrograph of SS $316 \mathrm{~L}$ control coupon taken at a magnification of $260 \mathrm{x}$. . . . . . . . . . 59

5. Scanning electron micrograph of SS $316 \mathrm{~L}$ control coupon

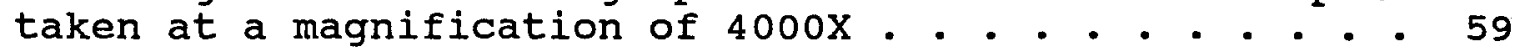

6. Scanning electron micrograph of SS $316 \mathrm{I}$ test coupon after exposure to brine and condensate taken at a magnification of $260 \mathrm{x}$. . . . . . . . . . . . . 60

7. Scanning electron micrograph of SS $316 \mathrm{~L}$ test coupon after exposure to brine and condensate taken at a

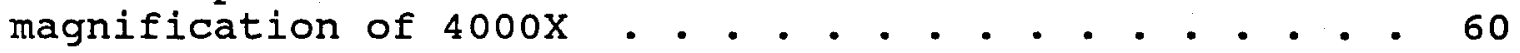

8. Scanning electron micrograph of SS $316 \mathrm{~L}$ test coupon after exposure to brine, condensate, and non-condensable

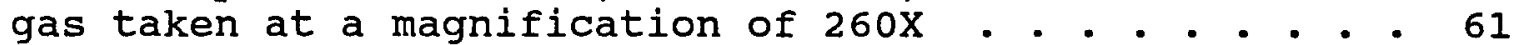

9. Scanning electron micrograph of SS 316L test coupon after exposure to brine, condensate, and non-condensable

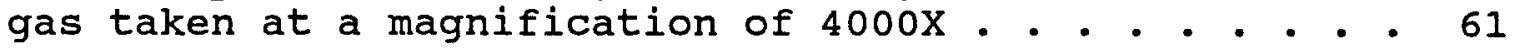


SECTION 1

INTRODUCTION

\subsection{GENERAL STATEMENT}

Dissolved silica $\left(\mathrm{SiO}_{2}\right)$ is a ubiquitous component of geothermal fluids, and its presence at elevated concentrations is routinely used as a chemical indicator of subsurface geothermal reservoir temperatures (White et al., 1956; Fournier and Truesdell, 1970, Fournier, 1981). The characteristics of dissolved silica that permit its use in this application are its: temperature dependent solubility; rapid equilibration to reservoir temperatures; and very slow precipitation from solution under typical pH conditions of naturally cooled geothermal fluids (Fournier and Rowe, 1977; Fournier and Truesdel1, 1970). These solubility characteristics can also generate severe operational and maintenance problems for any geothermal facility that must process geothermal fluids from a high temperature, water-dominated reservoir. Geothermal facilities that have encountered such problems include several in North and Central America (Imperial Valley, Cerro Prieto, Ahuachapan), in Iceland and Greece, and in the Pacific Basin (the Kilauea East Rift Zone of Hawaii, Japan, New Zealand, and the Philippines). The present report will describe a number of experiments that were performed at the HGP-A geothermal facility that evaluated methods of controlling the deposition of silica from the Kilauea geothermal fluids and of recovering silica as a potentially marketable byproduct. The ultimate objective of this work was to identify technically and economically viable methods of protecting geothermal brine handling equipment from excessive silica scale deposition and to allow liquid wastes from geothermal power generation to be disposed of in an environmentally acceptable manner.

\subsection{BACKGROUND}

The HGP-A well, drilled on the kilauea East Rift zone (KERZ) 
of the Island of Hawaii (Fig. 1) in 1976, encountered geothermal reservoir temperatures approaching $360^{\circ} \mathrm{C}$ at depths of $1960 \mathrm{~m}$.

Early testing of the well showed that the reservoir fluids were of modest salinity but had silica concentrations of nearly $500 \mathrm{mg} / \mathrm{kg}$ (Kroopnick et al., 1980) that, after flashing, increased to more than $850 \mathrm{mg} / \mathrm{kg}$. An effort to demonstrate the technical feasibility of producing electrical power from the geothermal resource discovered by this well was undertaken by a consortium of Federal, state, and county agencies and the local utility who installed a small wellhead generator facility on the HGP-A well in 1981 . The facility operated for eight years and enabled the plant operators and outside researchers to identify, and development management strategies for, a wide array operational and maintenance problems posed by the use of silica-rich geothermal fluids for power generation.

One of the most difficult maintenance problems encountered was associated with the treatment and disposal of the waste geothermal brines generated by the well. The high silica concentration in the brine, and its highly variable rate of deposition, resulted in silica scale formation throughout the brine system. An initial analysis of the operational and maintenance requirements of the facility, conducted in 1983 (Baughman et al., 1984), indicated that silica deposition tended to be minor in the mixed phase (steam/brine) system (Fig. 2) but increased in the brine disposal system downstream of the plant separator. The most critical areas for silica deposition were in and near valves in the brine piping, in the flash chamber immediately after discharge of the brine to atmospheric pressure, and in the brine disposal ponds. These deposition characteristics required redundant brine disposal systems, frequent repair and replacement of valves, and frequent servicing of the disposal ponds to remove accumulated silica sludge.

As a result of these problems, several preliminary studies were performed on the dissolved silica in an effort to better 


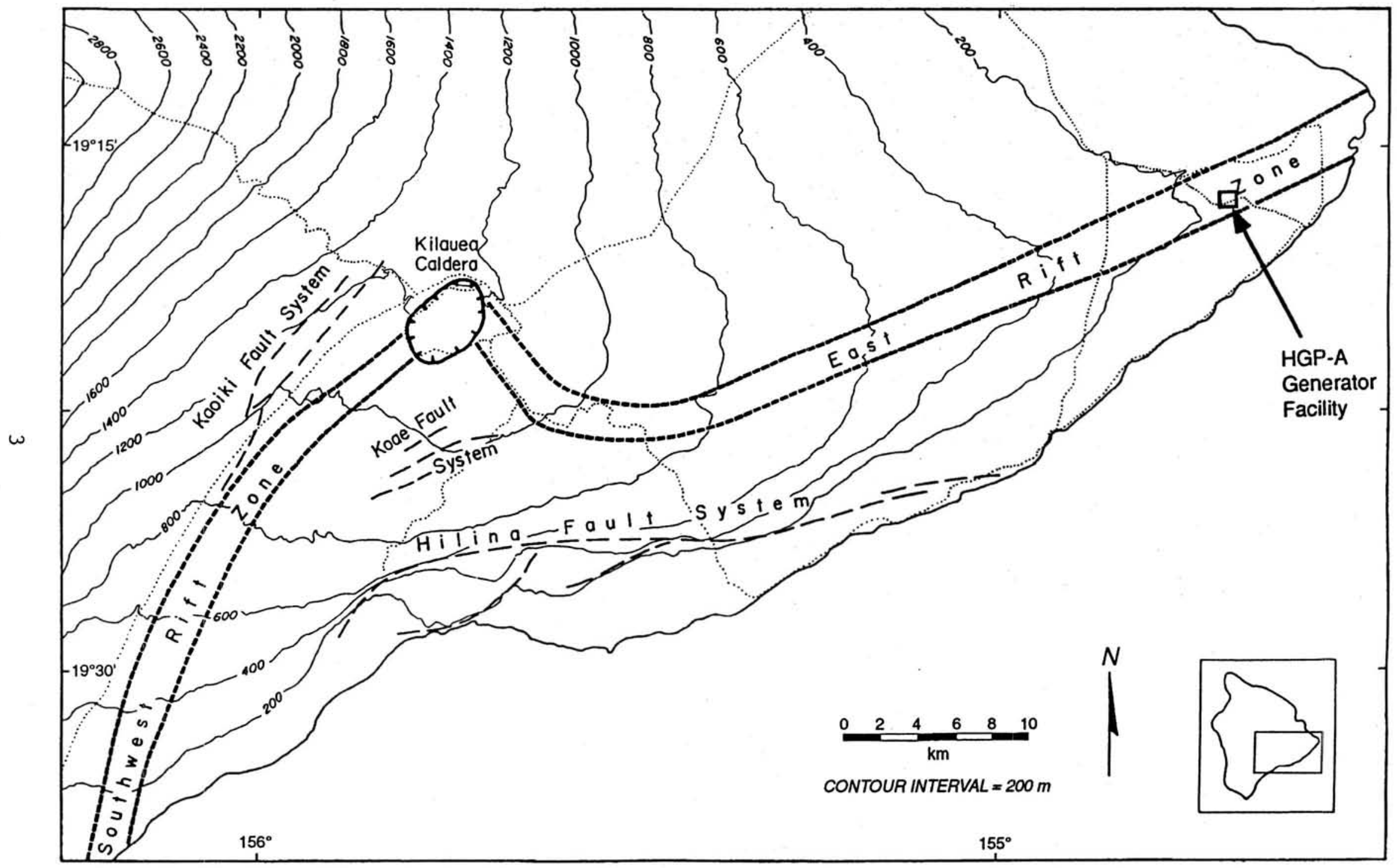

Figure 1. Location of the HGP-A Geothermal Generator Facility and Kilauea East Rift Zone hydrothermal system. 


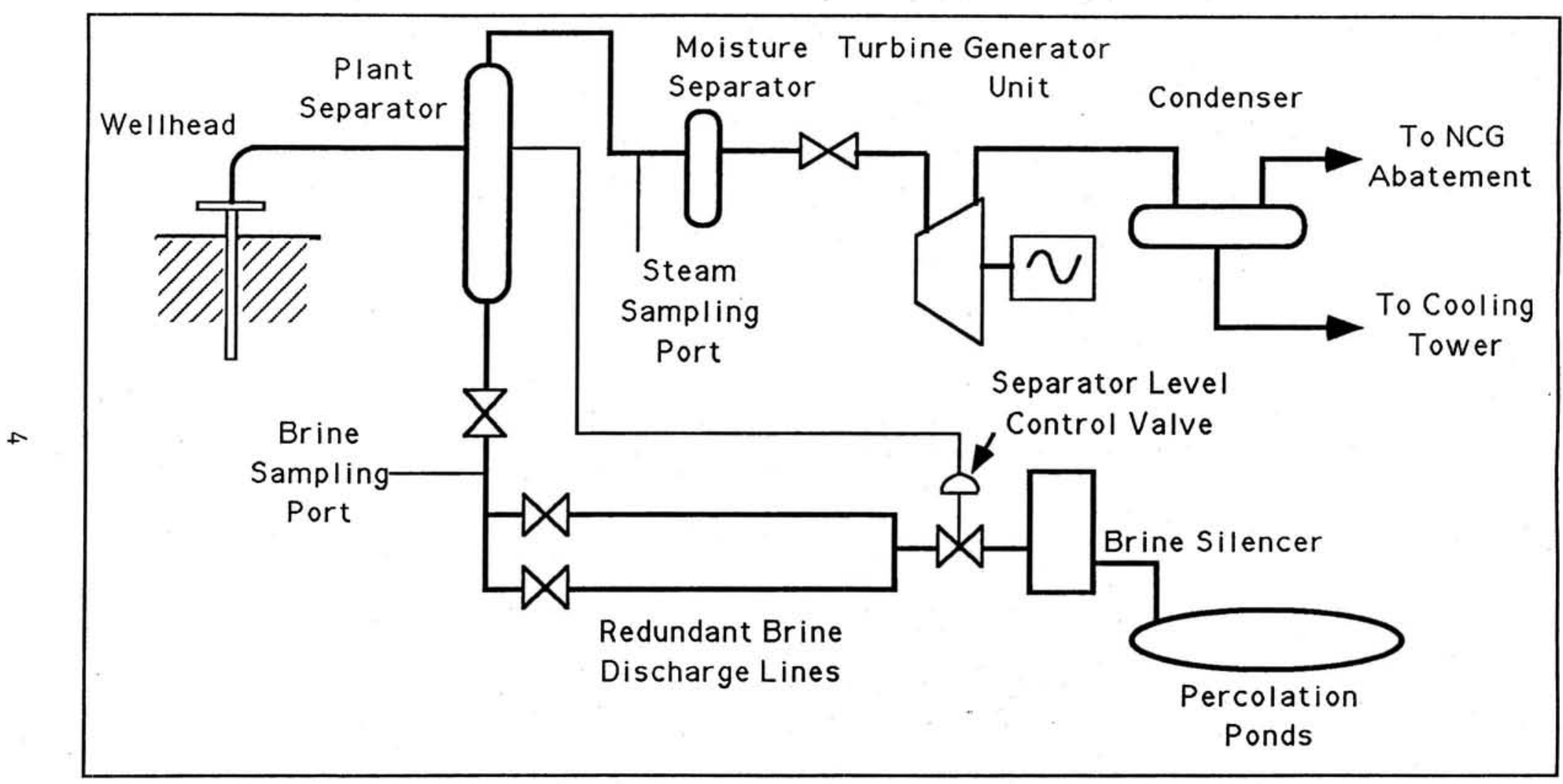

Figure 2. Line diagram of HGP-A Geothermal Generator Facility process flow. 
understand its chemistry and its response to changing chemical conditions in the geothermal brine. These studies showed that the precipitation of silica from the brines was highly pH dependent and suggested that recovery of a marketable silica byproduct was feasible as a means of removing silica from the geothermal fluids. This work also indicated that inhibition of silica deposition by proper pH control could enable a geothermal operator to dispose of the waste fluids by reinjection. The present investigation of methods of recovery and control of silica deposition from these fluids was undertaken in an effort to identify specific methods of silica control that will permit commercial geothermal generation facilities to dispose of their brines in an economically and environmentally acceptable manner.

\subsection{EXPERIMENTAL OBJECTIVES}

The research program was designed to identify an optimal method of silica control in Hawaii's geothermal fluids and included the following studies (see Appendix A for a detailed statement of work) :

a) Analysis of the rate of polymerization of silica from the geothermal fluids under sub-boiling conditions at a range of $\mathrm{pH}$ values and with the addition of retarding and accelerating agents;

b) Treatment of a continuous side-stream of sub-boiling brine with $\mathrm{pH}$ control and silica precipitation agents and: determination of optimal addition rates of each; analysis of the depositional characteristics of the precipitated silica; and evaluation of the efficiency of the removal process;

c) Fabrication of a bench-scale brine treatment system that will allow continuous flow testing of silica inhibition and acceleration reagents on geothermal brines under power plant temperature and pressure conditions;

d) Analysis of fluids discharged from the continuous flow treatment system for particle settling rates and fouling rates of filtration systems;

e) Development of a conceptual design for a larger pilot scale 
brine treatment system based on the results of the initial high temperature treatment system;

f) Analysis of the physical and chemical characteristics of the silica solids recovered from the geothermal brines and attempt to identify a silica recovery methodology that will provide the highest value by-product.

g) Preliminary evaluation of the chemical characteristics of geothermal fluids from North American geothermal fields to assess the applicability of the brine treatment methods developed at HGP-A to their brine disposal systems.

The following report reviews the results of these investigations and describes both a method for recovery of geothermal silica having a high surface area and a method of inhibiting silica precipitation to enable geothermal waste fluids to be reinjected back into the subsurface formation. It should be noted here, however, that premature termination of operations at the HGP-A geothermal facility, coupled with delays in receipt of funding, limited the time available to several of the field experiments that required continuous flow of geothermal fluids. Hence, we were unable to pursue some of the proposed studies to the extent initially planned by the investigators. 


\section{SECTION 2}

SILICA PRECIPITATION STUDIES

\subsection{POLYMERIZATION}

The first series of experiments evaluated the polymerization and precipitation rate of silica in the geothermal fluids. The first of these was an analysis of the polymerization rate of silicic acid, or silica monomer, to form suspended colloidal polymeric silica in solution.

\subsubsection{ExperimentaI Procedure}

This work consisted of a series of batch experiments using fluid samples at ambient temperature $\left(30^{\circ} \mathrm{C}\right)$. Samples of geothermal fluid from the power plant brine system were withdrawn from the brine system immediately downstream of the separator (Fig. 2) through a water-cooled stainless steel coil. The brine temperature and pressure at this sampling point were $188^{\circ} \mathrm{C}$ and $12.1 \mathrm{bar}$. The bulk sample was held in an insulated polyethylene container and aliquots of fluid were periodically removed over a span of 20 hours and analyzed using the molybdate yellow method (Strickland and Parsons, 1972). This analytical procedure is sensitive to the reactive silica monomer but is insensitive to silica that has polymerized to an oligomer form. The experiments were conducted at brine $\mathrm{pH}$ values of 7 , the natural $\mathrm{pH}$ of the unflashed brine, and with the $\mathrm{pH}$ of the brine adjusted to a value of 8 .

\section{1 .2 Results}

The results of a typical series of analyses (Fig. 3) show that there is a steady decline in the concentration of silica monomer from its initial value of $850 \mathrm{mg} / \mathrm{kg}$ down to a value of $200 \mathrm{mg} / \mathrm{kg}$ and to $250 \mathrm{mg} / \mathrm{kg}$ for the $\mathrm{pH} 8$ and $\mathrm{pH} 7$ brines respectively. In both cases, the reduction of monomer concentration occurs with no visible evidence of solid precipitation or gel formation in the fluid phase. According to Iler (1979), the absence of visible evidence of precipitation is due to the formation of a silica 


\section{Silica Polymerization Versus $\mathrm{pH}$}

$\infty$

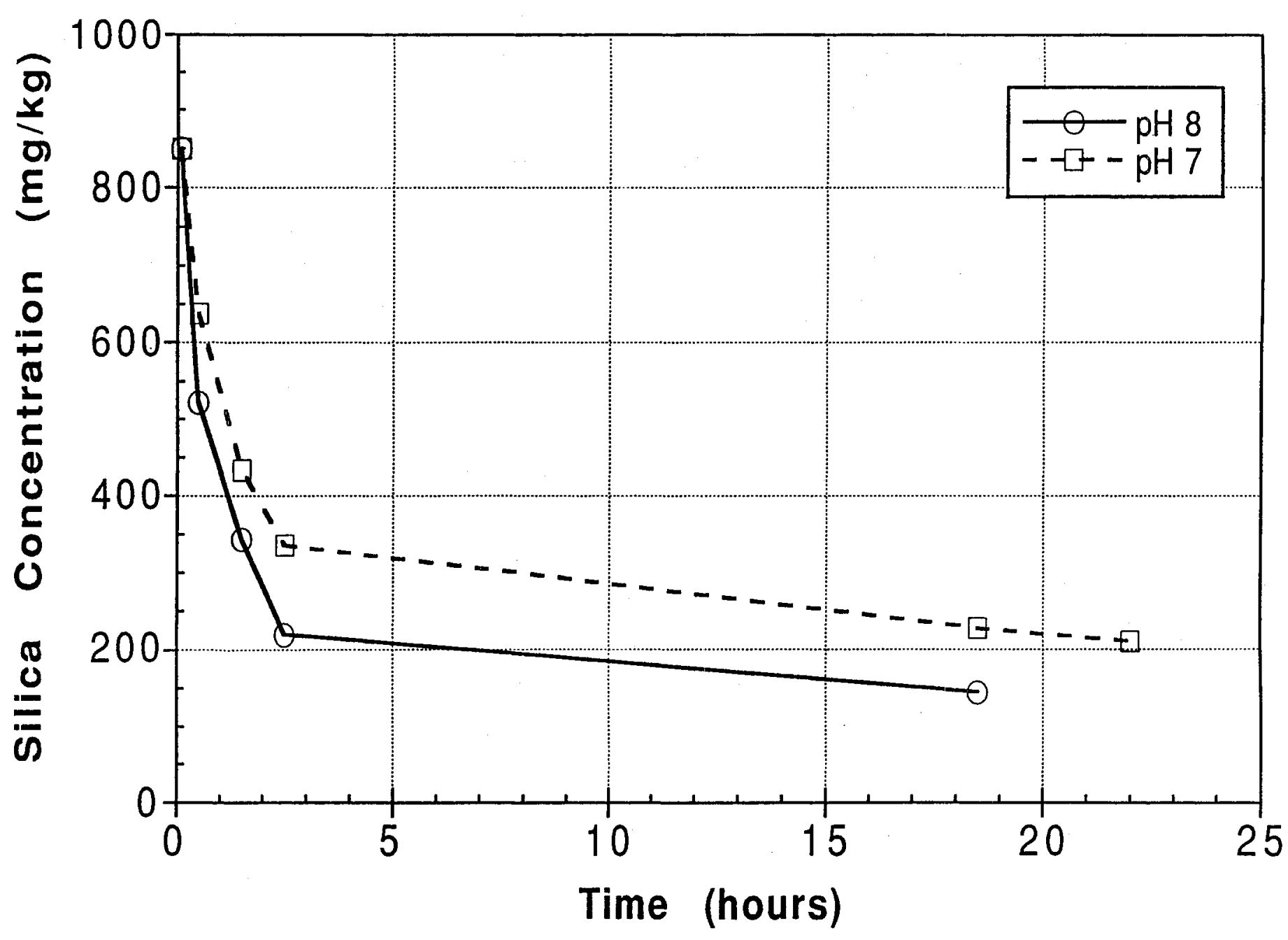

Figure 3. Decline in molybdate reactive silica concentrations with time. 
polymer sol that has a density and refractive index similar to that of water and is detectable only by ultra filtration of the sample. 2.1 .3 Analysis

The silica polymerization characteristics observed are, in some respects, similar to those that have been observed in other laboratory and field studies but also show some important differences from these studies. The most apparent difference is that there is no evidence of a delay, or critical nucleation, time for the silica prior to the onset of polymerization and decline in monomeric silica. Such delays have been observed in several studies (Weres et al., 1980; Weres and Tsao, 1981; Makrides et al., 1980; White et al., 1956) and is generally attributed to the differing solubility characteristics of varying sizes of silica polymers: smaller diameter polymers are significantly more soluble than larger ones. Hence, formation of sufficient numbers of nucleation centers within the fluid will require some period of time that is a function of the level of supersaturation and composition of the other dissolved constituents in the brine phase (including $\mathrm{pH}$ ). The absence of a detectable nucleation time in the HGP-A brines may have been the result of the wide sampling applied in our analytical methods. It is, however, more probable that flashing of the geothermal fluid in the reservoir (i.e. steam is separated from the brine in the formation) has enabled the brine to have an extended period of silica supersaturation (and nucleation) by the time the fluids were sampled at the separator discharge.

Analysis of the decline curve for the monomeric silica concentrations indicates that neither curve fits a first order kinetic equation of the form:

$$
-d c / d t=k A_{s}\left(c-c_{e}\right)
$$

where $c$ is the silicic acid concentration at time $t, c_{e}$ is the silica solubility, $A_{s}$ the silica surface area per volume of solution, and $k$ is the global rate constant (Flemming, 1985). Curvature of the $\log \left(c-c_{e}\right)$ versus time plot (Fig. 4) indicates that the rate of polymerization is not first order in the level of 


\section{Log of Silica Concentration Versus Time}

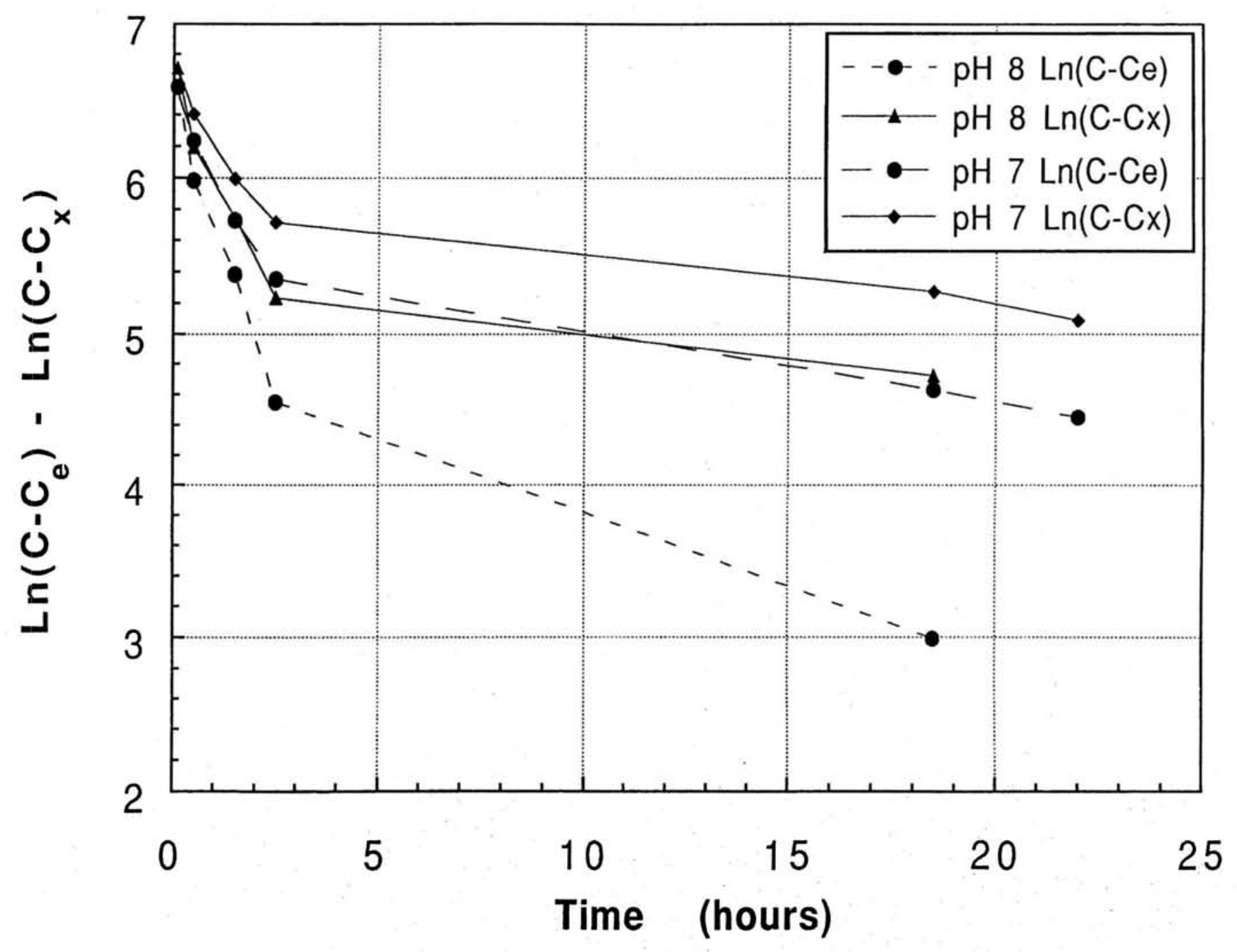

Figure 4. Analysis of silica decline curves following Flemming's (1986). suggested procedure. 
supersaturation of silica; similar analyses show that neither the rate expressions proposed by Weres et al. (1980) nor Goto (1956) adequately describe the polymerization process in our fluids. Although Flemming suggests that the polymerization rate expression should be rewritten as:

$$
-d c / d x=k\left(c-c_{x}\right)
$$

where $c_{x}$ represents a psuedo-equilibrium of silicic acid with a silica surface that is saturated with chemisorbed silicic acid. calculation of the psuedo-equilibrium concentrations using his method did not yield appreciably better results (Fig. 4), suggesting that the monomer decline reflects both nucleation and particle growth in the supersaturated brines.

The most important result of these experiments for our purposes is that the rate of removal of silicic acid is more rapid at the higher $\mathrm{pH}$. Acceleration of the polymerization reaction may be attributable to hydroxyl catalyzation: Because silica is a weak acid, increased hydroxyl ion concentrations increase the degree of ionization of silicic acid silanol groups as follows:

$$
\mathrm{H}_{4} \mathrm{SiO}_{4}+\mathrm{OH}^{-} \rightarrow \mathrm{H}_{3} \mathrm{SiO}_{4}^{-}+\mathrm{H}_{2} \mathrm{O} \text {. }
$$

The increased concentration of the silicate ion in solution will, in turn, increase the rate of formation of the oligomer. Increasing the fluid $\mathrm{pH}$ has a further effect on the silica polymer -- at progressively higher $\mathrm{pH}$, the silica surface is capable of undergoing rapid ion exchange with the dissolved ions present in solution. Increased ion exchange has a strong effect on the critical coagulation concentration (c.c.c.) of dissolved ions for suspended silica colloid (Allen and Matijavic, 1969, 1970, 1971; Allen et al., 1971; Iler, 1975) and has also been proposed as a means of increasing the rate of growth of the silica polymers (Iler, 1979). Calcium and other divalent cations have been shown to have a strongly pH-dependent influence on silica solubility by acting as bridging ions between the silica surface and added silicic acid ions as they attach to the polymer and thus increase the rate of polymer growth and agglomeration (Allen and Matijavic, 
1969, 1970, 1971; Iler, 1975, 1979). The moderate concentrations of calcium in the geothermal fluids from the HGP-A well (Table 1), indicate that calcium ion exchange plays a significant role in the silica deposition characteristics observed.

A second series of experiments was attempted at both higher pH values and with metal ion reagent additions in an effort to further explore the interaction of silicic acid with precipitating reagents. The planned procedure was to adjust the pH or metal ion reagent in the cooled brine to a predetermined concentration and to periodically withdraw fluid samples for silica analysis using a Technicon auto analyzer. This instrument is capable of performing silica analyses using an automated flow-through system that conducts repeated coloremetric analyses at very closely spaced time intervals. In our initial attempts to use this system, however, we found that the analytical data were highly variable and were inconsistent with silica analyses performed using the standard molybdate method. In spite of attempts to improve our analytical accuracy, we were unable to develop a methodology that gave acceptable results. An effort was also made to characterize the $\mathrm{pH}$ dependence of polymerization using standard methods but the rates became so rapid that we were unable to reproducibly "quench" the polymerization process to make accurate determinations of monomer concentrations. Hence, more detailed analyses of silica precipitation rates were deferred in order to evaluate other aspects of the silica precipitation process. The experiments conducted at high $\mathrm{pH}$ did show, however, that further increases in $\mathrm{pH}$ resulted in substantially higher polymerization rates and a rapid formation of a gelatinous floc that precipitated at varying rates from the brine solution.

\subsection{PRECIPITATION}

2.2.1 Experimental Procedure

A second series of batch tests was performed to evaluate the effect of $\mathrm{pH}$ on the settling rates of silica from solution as well as the efficiency of silica removal. In these experiments, samples 


$\begin{array}{lclc}\mathrm{Cl} & 9802 & \mathrm{H}_{2} \mathrm{~S} & 820 \\ \mathrm{Li} & 1.08 & \mathrm{CO}_{2} & 975 \\ \mathrm{Na} & 5143 & \mathrm{~N}_{2} & 120 \\ \mathrm{~K} & 748 & \mathrm{H}_{2} & 12 \\ \mathrm{Ca} & 515 & & \\ \mathrm{Mg} & 0.264 & \text { Physical } & \text { Parameters } \\ \mathrm{SO}_{4} & 14.4 & & 187^{\circ} \mathrm{C} \\ \mathrm{SiO}_{2} & 853 & \text { Temperature } & 12 \mathrm{Bar} \\ \mathrm{Fe} & 0.20 & \text { Pressure } & \\ \mathrm{Pb} & 0.46 & & \\ \mathrm{Zn} & 0.07 & & \end{array}$

of cooled $\left(30^{\circ} \mathrm{C}\right)$, filtered, brine were placed in a $75 \mathrm{~mm}$ diameter by $750 \mathrm{~mm}$ long glass cylinder. To this solution was added, under rapid stirring, a pre-determined volume of $4 \mathrm{~N}$ hydrochloric acid or sodium hydroxide solution to yield the desired pH. After complete mixing of the acid/caustic with the brine, the solution was permitted to stand undisturbed while the solids formed settled to the bottom of the container. During the settling process, the volume occupied by the settled floc was recorded as a percent of the total solution volume. After the solids had settled for 30 minutes or longer, samples of the solution were recovered, filtered, and diluted for analysis of the residual total silica (monomer and oligomer) by atomic absorption analysis.

\section{2 .2 Results}

The results of these experiments (Figs. 5, 6, and 7) indicate that silica precipitation and settling are not simple functions of $\mathrm{pH}$. At low pH values, silica does not agglomerate from solution and there is no silica precipitate formed even after periods of several hours. Analysis of residual silica shows that, even at very low $\mathrm{pH}$ values, less than $3 \%$ of the silica is removed from the monomer/oligomer form in solution (Fig. 5).

At $\mathrm{pH}$ values slightly above that of the untreated brine ( $\mathrm{pH}$ 9.3, Fig. 6), silica agglomeration and settling occurred very 
Total Silica Versus pH

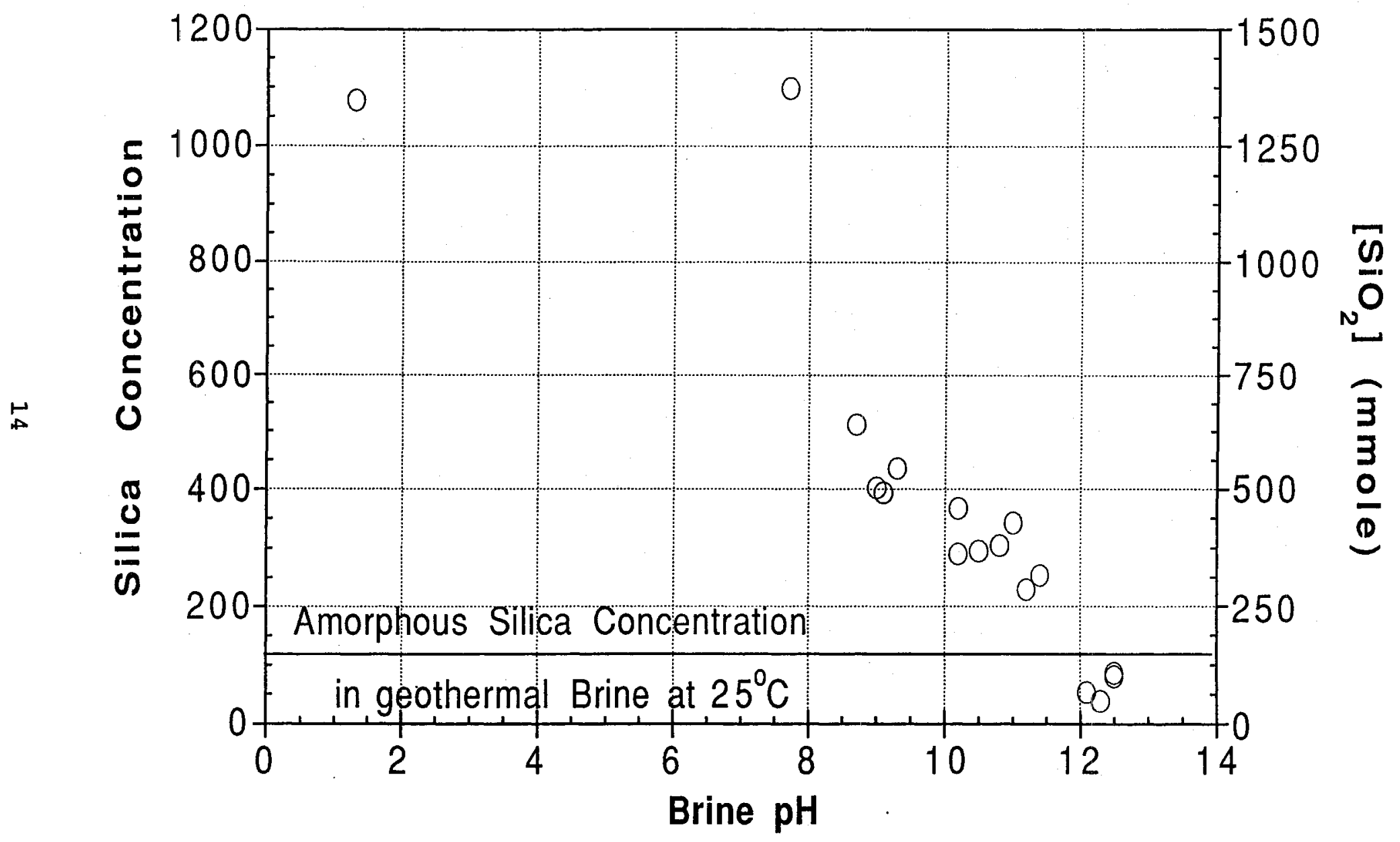

Figure 5. Residual total silica present in geothermal brines as a function of $\mathrm{pH}$ of treatment. 


\section{Settling Volume of Silica Flocculate}

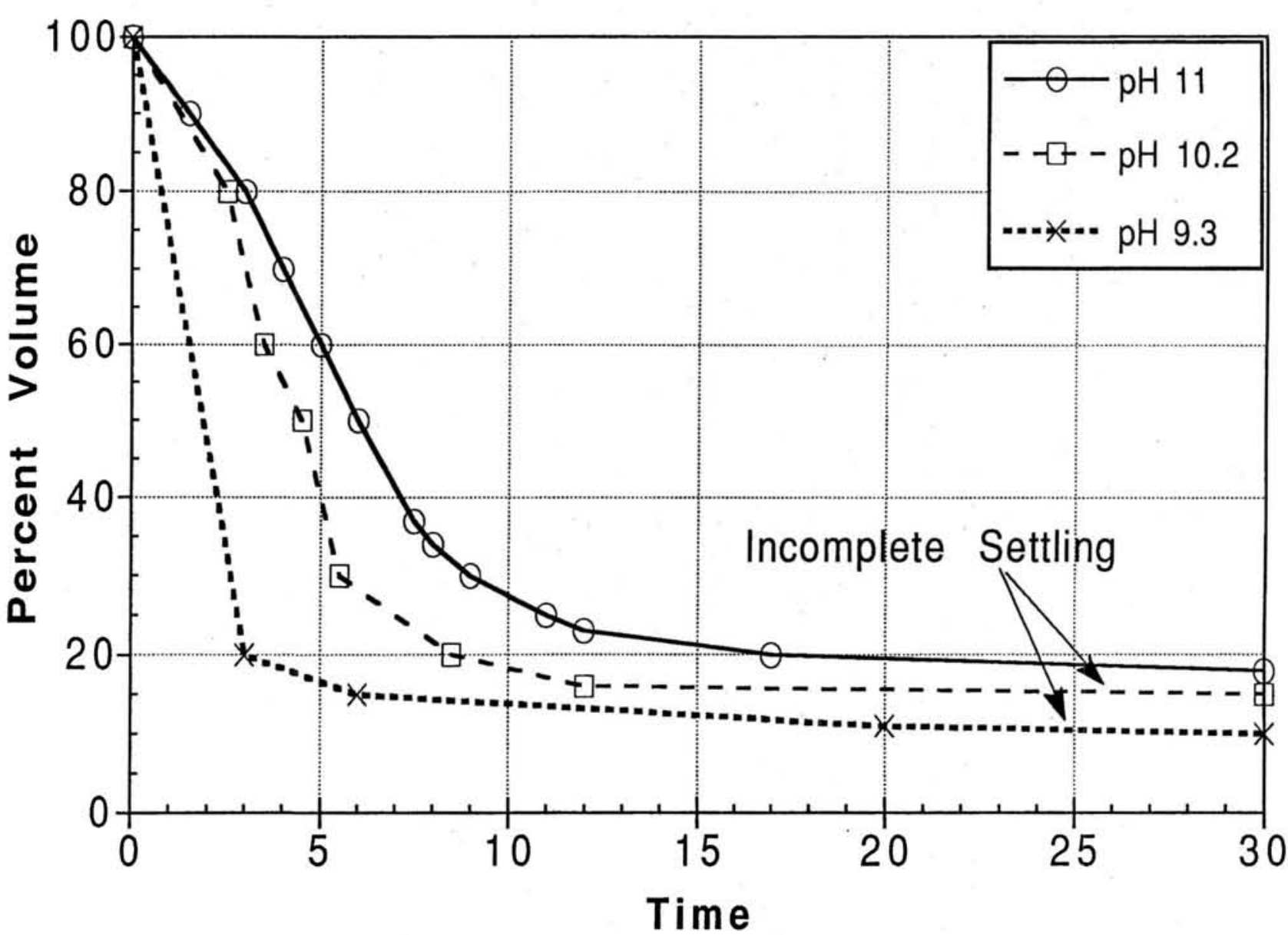

Figure 6. Rate of settling of agglomerated silica floc as a function of $\mathrm{pH}$ : intermediate $\mathrm{pH}$ treatments. 
Settling Volume of Silica Flocculate

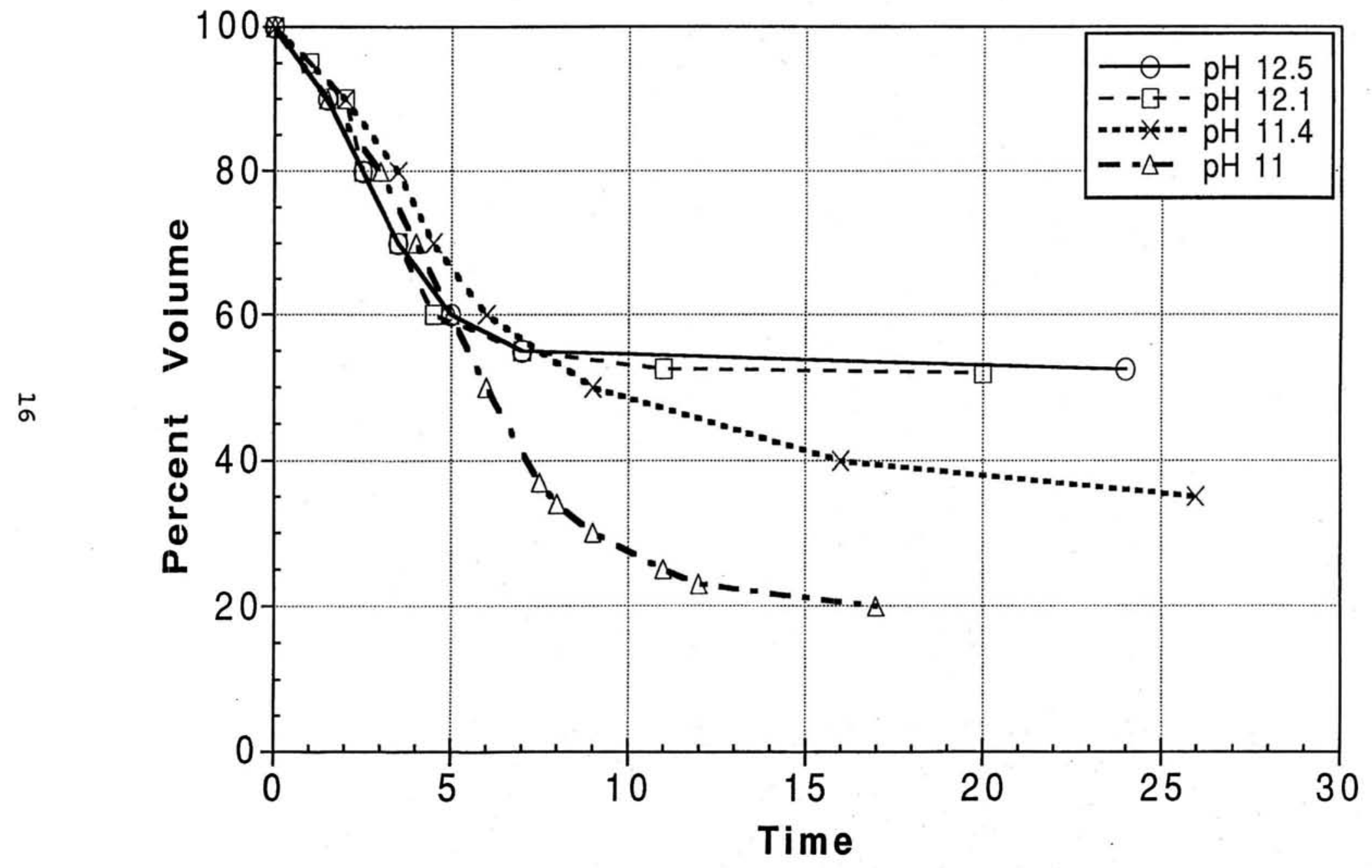

Figure 7. Rate of settling of agglomerated silica floc as a function of $\mathrm{pH}$ : intermediate $\mathrm{pH}$ treatments. 
slowly and built up only a small accumulation of silica floc at the bottom of the cylinder. Most of the agglomerated floc remained suspended in the solution even after an extended settling time. Analysis of the residual silica present in solution showed that $30 \%$ to $40 \%$ of the silica remained as silicic acid monomer and oligomer (Fig. 5). Increasing the brine $\mathrm{pH}$ to 10 to 11 (Fig. 6), resulted in markedly increased precipitation and settling rates with the suspended solids removed from $80 \%$ of the liquid volume in less than 15 minutes. At these higher $\mathrm{pH}$ values, the residual silica that remains in solution as oligomer progressively decreases from $30 \%$ to about $20 \%$ of that originally present.

As the $\mathrm{pH}$ values are increased to above 11, the precipitation and settling rates remain high, but the final volume occupied by the solids increases substantially (Fig. 7). The residual silica concentrations in the supernatant, however, continue to decrease to concentrations of about 20 to $40 \mathrm{mg} / \mathrm{kg}$ or about $2 \%$ to $5 \%$ of the total silica initially present in the brine phase (Fig. 5).

Samples of the solids from these precipitation experiments were recovered for analysis of their surface charge (zeta potential) at the $\mathrm{pH}$ of precipitation. These measurements, presented in Table 2 and Figure 8, show that the zeta potential of the silica floc carries a strong negative charge at the lower and intermediate portion of the precipitation range, but this charge progressively weakens as $\mathrm{pH}$ is increased until a point of zero charge $(\mathrm{PZC})$ is reached at $\mathrm{pH} 11.5$ to 11.8 . Above this $\mathrm{pH}$ the silica particles attain a positive surface charge that appears to increase with increasing $\mathrm{pH}$.

\section{2 .3 Analysis}

Several aspects of these results are deserving of comment. The residual silica concentrations found in the high $\mathrm{pH}$ precipitation experiments are well below the solubility of amorphous silica in this $\mathrm{pH}$ range, calculated to be in excess of $1000 \mathrm{mg} / \mathrm{kg}$ at $\mathrm{pH} 11$ (Iler, 1979; Morey et al., 1964; Okamoto et al, 1957), and are even lower than the solubility of silica in pure water under neutral pH conditions $(128 \mathrm{mg} / \mathrm{kg}$, Fournier and Rowe, 


\section{Zeta Potential of Silica Floc Versus pH}

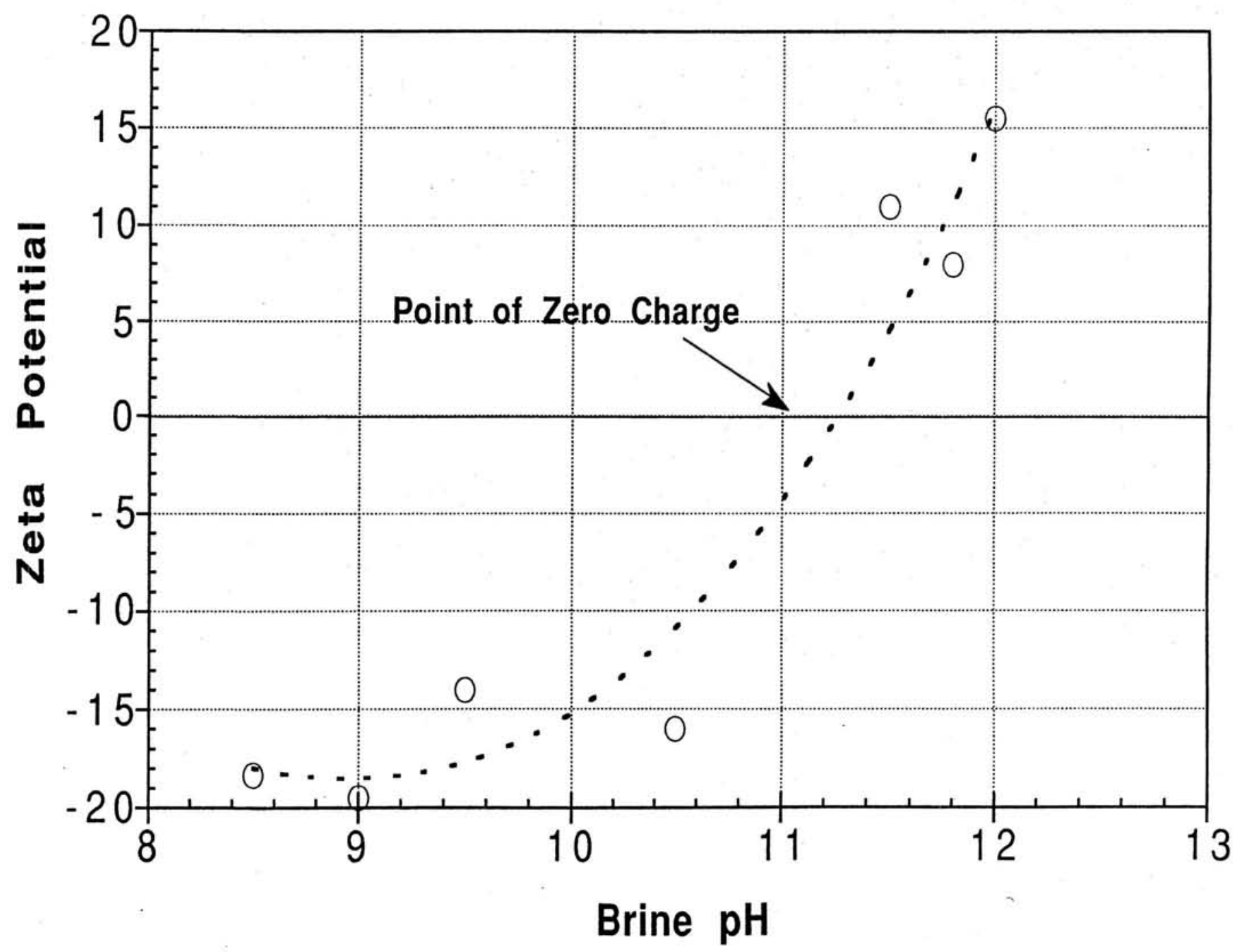

Figure 8. Zeta potential of recovered silica floc as a function of $\mathrm{pH}$ of precipitation 


$\begin{array}{llllllll}\begin{array}{l}\text { PH of } \\ \text { Precipitation }\end{array} & 8.5 & 9.0 & 9.5 & 10.5 & 11.5 & 11.8 & 12.0 \\ \text { Zeta Potential } & -18.3 & -19.5 & -14 & -16 & +11 & +8 & +15\end{array}$

1977). The presence of a PZC for the amorphous silica at these elevated $\mathrm{pH}$ levels is also contrary to the results of most prior investigations that report strong negative surface charges on the silica polymers at high fluid pH (Allen and Matijevic,1969; Bolt, 1957; Iler, 1979; Parks, 1965).

The silica responses observed arise from the high ion exchange capacity of silica and its strong dependence on fluid pH. A number of studies have shown that silica is highly susceptible to ion exchange in aqueous electrolytes (Bolt, 1957; Carroll and Freeman, 1954 ; Chen and Marshall, 1982 ; Iler, 1975; Marshall, 1980; Marshall and Chen, 1982; Marshall and Warakomski, 1980) and that the rates of ion exchange are highly $\mathrm{pH}$ dependent: As $\mathrm{pH}$ is increased, the ionization of silanol groups on the surface of the silica polymers permits cation exchange with the ionized surface. The ion exchange effect has been found to be substantialiy stronger for doubly ionized cations (e.g. calcium ion) and Iler (1979) has suggested that the calcium ion can act as a bridge for the attachment of silicic acid to the polymer surface; Yokoyama et al. (1989) suggest a similar mechanism for the effect of aluminum ion on silica solubility. An analysis of the precipitation kinetics of silicic acid by Flemming (1986) proposed that the initial polymerization is governed by a psuedo-equilibrium solubility of silicic acid with the polymer surface saturated with chemisorbed silicic acid. Under our experimental conditions of high $\mathrm{pH}$ and moderate concentrations of dissolved ions (Table 1), the silica surface is saturated with chemisorbed silicic acid and bridging cations that represent a much more favorable depositional environment than silica polymer in pure water. Hence, the initial psuedo-equilibrium concentration of silicic acid under our 
experimental conditions is well below that for amorphous silica in pure water. Whether the silica concentrations would increase in our $\mathrm{pH}$ modified brine to higher values after surface rearrangement and dehydration of the silica polymers, as suggested by Flemming (1986), was not determined.

The appearance of a positive surface charge on the silica is also attributed to ion exchange processes occurring on the polymer surface. The presence of moderate concentrations of calcium ion (ca. 10 mmolar) in the brine enables a progressively larger number of calcium ions to attach to the silica surface as $\mathrm{pH}$ increases. Because calcium is doubly charged, the negative surface charges are increasingly counterbalanced by calcium ion exchange until a net positive charge is generated at the silica surface.

The practical applications of these results can be summarized as follows:

a.) The presence of moderate to high concentrations of calcium in a silica-rich brine can result in rapid rates of silica precipitation. If silica removal from geothermal brines is to be done by precipitation, the above interpretation, as well as the work done by Iler (1975) suggests that one effective approach is to increase both the calcium ion concentration and the $\mathrm{pH}$ of the geothermal discharge brines. The combination of these changes will decrease the solubility of silica, increase its rate of precipitation, and increase the rate of agglomeration of the precipitated silica.

b.) If precipitation and settling of silica is the method of choice for control of silica deposition, the optimum pH for silica removal is in the range of 11.0 to 11.4 . This $\mathrm{pH}$ will give most rapid precipitation and minimum volume of the recovered silica floc that must be processed for dewatering.

\subsection{BATCH RECOVERY OF SILICA FROM GEOTHERMAL BRINES}

Another series of experiments evaluated the effect of adding transition metal ions to the brine to accelerate precipitation of amorphous silica from solution. These experiments were suggested by earlier research (Decarlo and Thomas, 1985; Decarlo and Ronay, 
1987; De Jong and Brown, 1980; Iler, 1979; Wirth and Geiskes, 1978; Yokoyama et al., 1989) that indicated that silica oligomers in pure water have a strong affinity for highly charged transition metal ions added to a colloidal suspension. The metal ions chosen for use in the present study were iron $\left(\mathrm{Fe}^{3+}\right)$ and aluminum ( $\left.\mathrm{Al}^{3+}\right)$; sources of both are readily available and relatively inexpensive as industrial chemicals (e.g. as $\mathrm{Fe}_{2}\left(\mathrm{SO}_{4}\right)_{3}$ and $\left.\mathrm{KAl}\left(\mathrm{SO}_{4}\right)_{2}\right)$.

\subsubsection{Experimental Procedure}

The first set of experiments evaluated the relative efficiency of iron and aluminum in removing silica from solution. A series of ten-liter samples of geothermal fluid were removed from the brine system immediately downstream of the plant separator. The fluids were withdrawn through a cooling coil that yielded samples at $30^{\circ} \mathrm{C}$ and a pH of approximately 6.5 to 7.0. Varying amounts of $\mathrm{Fe}_{2}\left(\mathrm{SO}_{4}\right)_{3}$ and $\mathrm{KAl}\left(\mathrm{SO}_{4}\right)_{2}$ were added to the brine samples, and the pH was adjusted to 8. The samples were stirred vigorously and permitied to settle for one-half hour. Samples of the supernatant were withdrawn, filtered, and diluted ten-fold for later analysis of residual silicic acid and suspended oligomer.

\section{3 .2 Results and Analysis}

our results (Fig. 9) show that the addition of quite low concentrations of either metal substantially reduces the residual silica concentrations in the liquid phase. The most striking aspect of the response is that there is a two- to three-fold reduction in residual silica concentrations at relative silica to metal ion molar concentrations approaching 50:1. The sensitivity of silica to the metal ion addition is believed to be associated with the ion exchange behavior of the surface silanol groups at elevated $\mathrm{pH}$ and to the high charge density carried by the multiplycharged metal ions in solution. Similar to the case for calcium ion coagulation of the silica, the charged metal ions act as bridging points for attachment of silicic acid monomer as well as other silica polymers. This is consistent with the results of Iler (1975, 1979), Rothbaum et al. (1979) and Yokoyama et al. (1989). 
Residual Silica Versus Metal Ion Concentration $(\mathrm{pH}=8)$

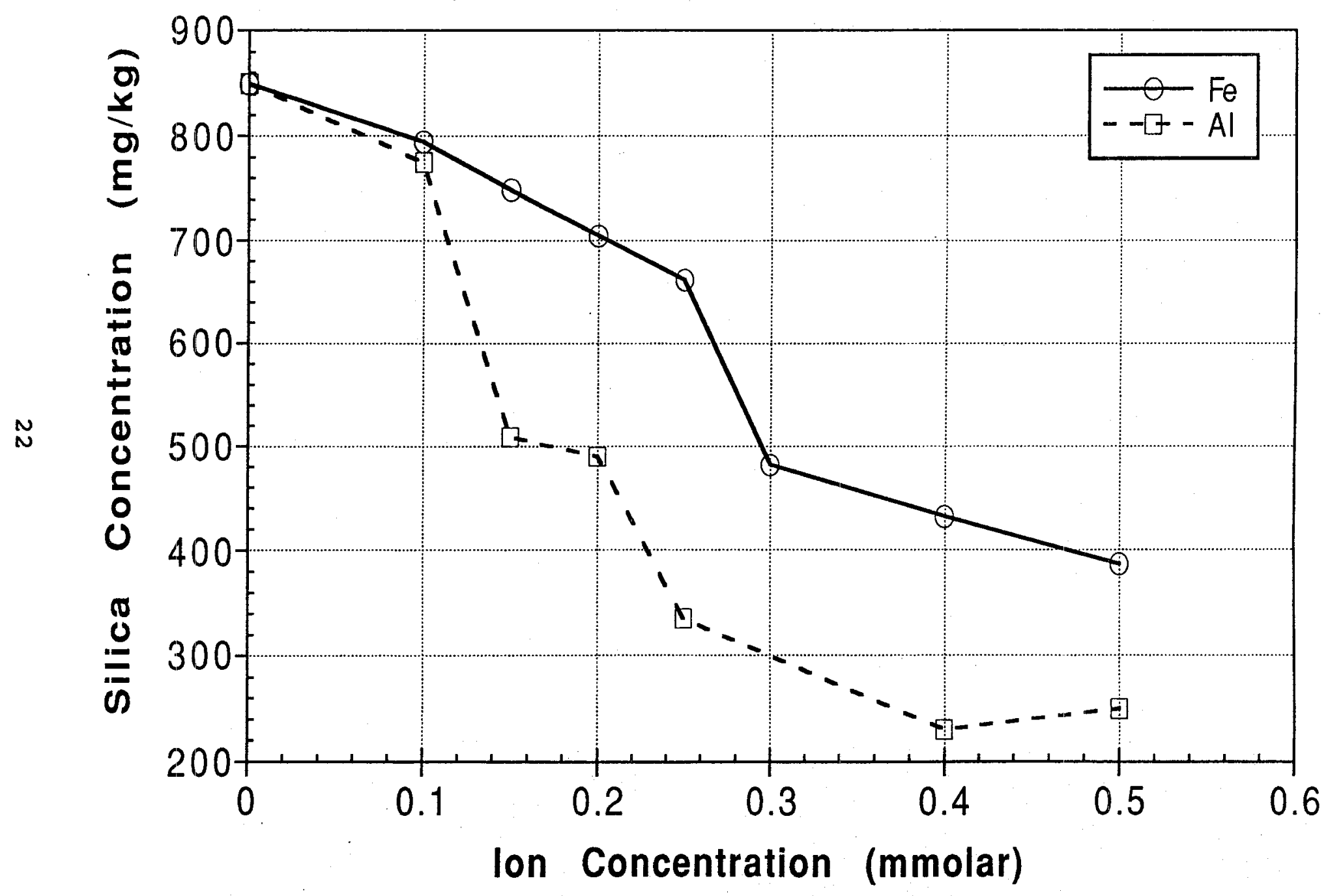

Figure 9. Residual total silica present in geothermal fluids as a function of iron and aluminum treatments. 
Our results also indicate an apparent difference in the relative efficiencies of iron and aluminum ions in their ability to precipitate silica polymer from solution. A second set of experiments, conducted on flashed and unflashed brines, shows that the addition of iron to the unflashed fluids require substantially more iron to precipitate the silica than those that have undergone a second, atmospheric, flashing process (Fig. 10 and 11). The difference between the two responses can be accounted for by the presence of sulfide ion in the unflashed brine which removes a significant portion of the iron in the form of $\mathrm{Fe}_{2} \mathrm{~S}_{3}$ before it can react with the silica polymer (Rothbaum et al., 1979). In flashed brine, the bulk of the sulfide has been removed in the secondary steam phase and hence cannot compete with the silica for iron. The formation of a sparse black precipitate when iron was added to the unflashed brines tends to confirm this interpretation.

The difference in the reactivities of aluminum ion with the silica present in flashed and unflashed brine (Fig. 11) also shows a slight increase in silica removal associated with the loss of the acid gases. Although in this case there is no loss of insoluble sulfide, the slight increase in $\mathrm{pH}$ brought about by the removal of the gases further enhances precipitation. similar boiling induced $\mathrm{pH}$ changes are believed to be responsible for the presence of enhanced silica precipitation in the brine handing system of the HGP-A Generator Facility. Neither time nor resources were available to further explore the mechanism of this sensitivity.

The effectiveness of the metal ion addition in removal of silica from the geothermal brines suggests that silica removal by addition of very low concentrations of multiply charged transition metal ions may enable us recover a higher purity silica byproduct from the geothermal brines than would normally be possible using a calcium ion or lime addition to the waste fluids from a geothermal facility. 
$\mathrm{Fe}_{2}\left(\mathrm{SO}_{4}\right)_{3}$ Catalyzed Silica Precipitation

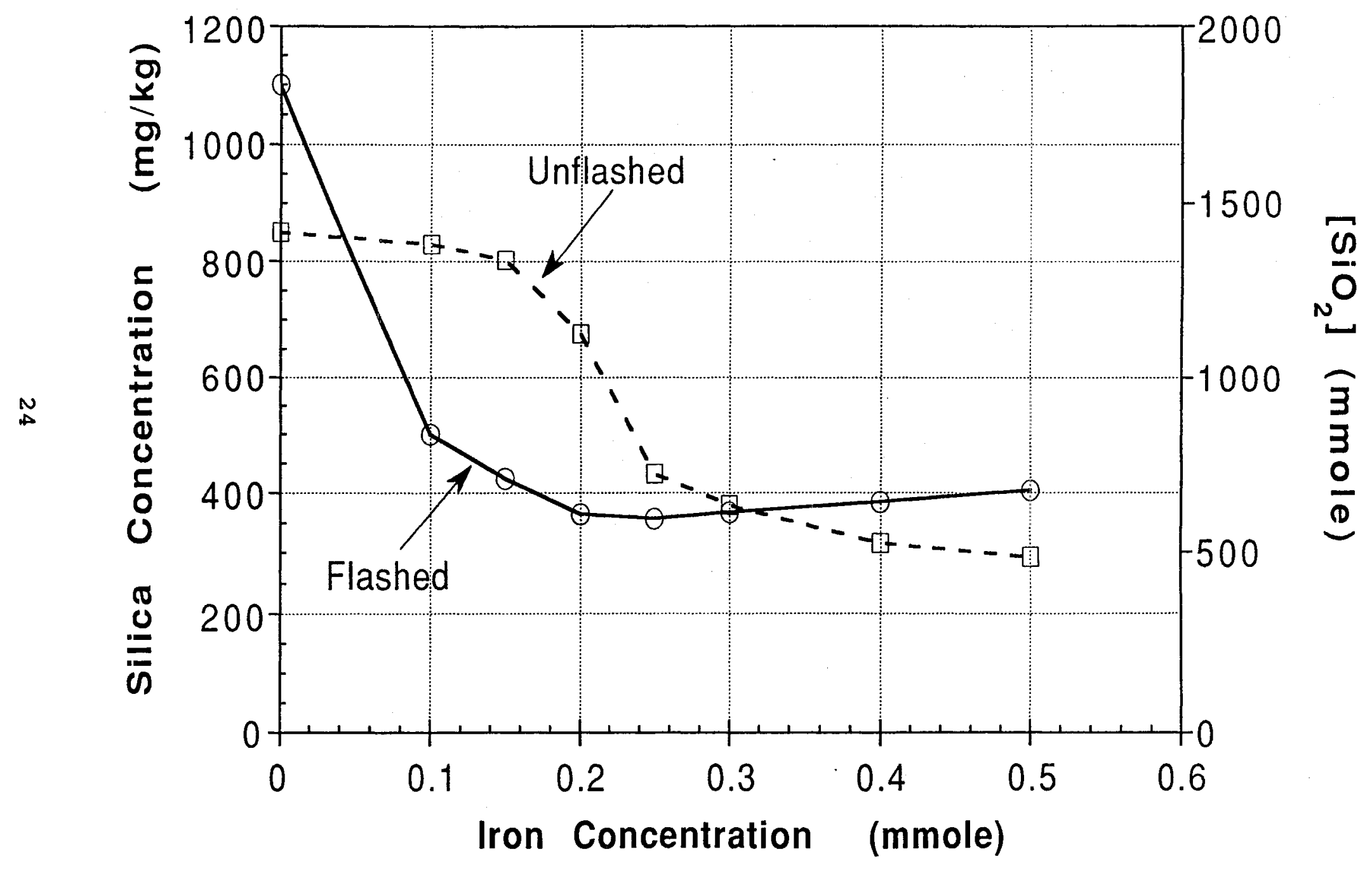

Figure 10. Residual total silica present in geothermal fluids as a function of iron addition for flashed and unflashed brines. 


\section{$\mathrm{Al}_{2}\left(\mathrm{SO}_{4}\right)_{3}$ Catalyzed Silica Precipitation}

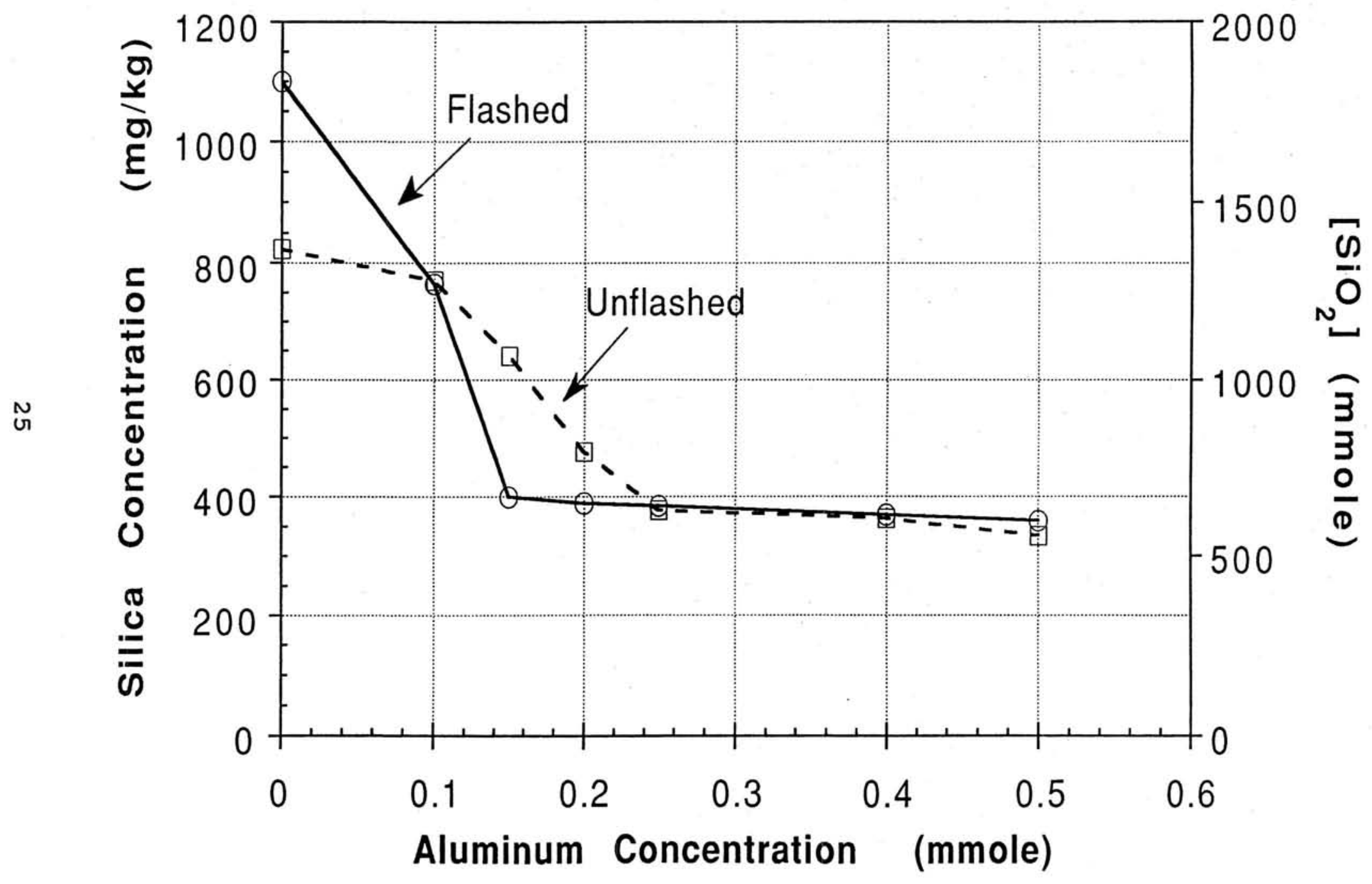

Figure 11. Residual total silica present in geothermal fluids as a function of aluminum addition for flashed and unflashed brines. 


\subsection{SUMMARY AND CONCLUSION}

The batch silica precipitation studies have shown:

a. There was no detectable nucleation time for silica polymerization from the geothermal fluids tested;

b. Increases in $\mathrm{pH}$ tend to destabilize colloidal suspensions of silica oligomer;

c. Surface charge effects play a principal role in the stability of the silica colloid: At low to intermediate pH, the high negative surface charge on the colloidal suspension inhibits oligomer growth and agglomeration. At high pH, enhanced ionization of the surface silanol groups promotes ion exchange with multiply charged cations in the solution. Attachment of cations to the silanol groups increases rates of polymer growth and cross linking of silica polymers, and progressively weakens and eventually reverses the surface charge on the suspended silica.

d. There is a strong affinity of silica oligomers for multiply charged transition metal ions. The sensitivity of the silica agglomeration process to metal ion concentrations of one to two orders of magnitude below that of suspended silica suggests that the metal ion acts through a bridging mechanism between silica oligomers having molecular weights on the order of 6000 or more.

\subsection{APPLICATIONS}

These findings suggest three approaches toward controlling silica precipitation in geothermal fluids:

a.) Addition of metal ion reagents and caustic soda or lime to increase the fluid $\mathrm{pH}$ and accelerate silica agglomeration followed by extraction of silica from the fluids or;

b.) Acidification of the brine to reduce ion exchange of the silica polymer and inhibit silica agglomeration to permit reinjection of the waste fluids into the subsurface. Acidification of the brine may also assist in the inhibition of silica deposition on pipelines and valves by reducing the concentration of silica polymer in suspension in the geothermal fluids. 
c.) Control of dissolved transition metal ion concentrations in the brine phase, possibly through the addition of sulfide ion, might be an effective means of inhibiting agglomeration of silica within the geothermal fluids. 
SECTION 3

SILICA RECOVERY INVESTIGATIONS

\subsection{BACKGROUND}

The above series of batch tests demonstrated that dissolved silica solids can be removed from Hawaii's geothermal brines to permit their reinjection into the subsurface formation; however, both the costs of removal and the means of disposal of the silica solids present substantial obstacles to this method of silica control. In an effort to address these issues, we investigated a series of variations of the basic recovery process in an effort to produce a silica byproduct that would have sufficient value to defray the costs of the control and recovery process.

The first step in the optimization process was to identify potential markets for silica and to determine their relative market values. Although silica is a widely used material, the majority of its use is as a bulk commodity for which its sales price varies in the range of about $\$ 90$ to $\$ 265$ per ton (Chemical Marketing Reporter, 1992). These applications include use in the manufacture of cement, glass, paper, rubber, and fertilizers and the characteristics required are relatively easily achieved. The low price and wide availability of these forms of silica, make it highly probable that the costs of recovery would be far higher than any reasonable sales price for the silica recovered. Hence, it was decided to pursue the development of a higher value silica product that is used in specialty chemical and processing applications for which the value would approach one dollar a pound. In discussions with representatives of chemical companies, we were informed that the key elements in the value of this product were particle size/surface area and product purity.

\subsection{OPTIMIZATION OF PARTICLE STZE/SURFACE AREA}

A series of experiments were performed that varied the method of recovery and the post-recovery treatment of the silica residue 
in an effort to increase surface areas and decrease particle sizes of the final product. The first experiments tested the response to differing metal ion addition ( $\mathrm{Fe}$ or $\mathrm{Al}$ ) and polymerization times after the sample was withdrawn from the brine system.

\subsubsection{Experimental Procedure}

Brine was withdrawn through a stainless steel heat exchanger, where its temperature was lowered to $30^{\circ} \mathrm{C}$, into a 101 polyethylene container where it was spiked with spiked with iron sulfate solution to yield an iron concentration of 0.5 molar. The solution was well mixed and a sufficient quantity of sodium hydroxide solution was added to increase the $\mathrm{pH}$ into the range of 10.5 to 11.0 . The treated samples were settled for 30 minutes, the bulk of the supernatant was pumped away, and the remaining gel layer was centrifuged at $900 \mathrm{rpm}$ for 20 minutes. The recovered gel was then given three treatments of a distilled deionized water wash followed by centrifugation after which the sample was dried at $110^{\circ} \mathrm{C}$ and stored in a sealed container. In subsequent extractions, untreated brine samples were allowed to polymerized for periods of 1,4 , and 16 hours prior to spiking and $\mathrm{pH}$ adjustment. The solids from these runs were recovered and washed in an identical manner to that described above. A second set of brine samples received identical treatment except that aluminum sulfate was used to spike the brine to a 0.5 mmolar concentration of $\mathrm{Al}^{3+}$.

After the samples were dried, dynamic surface area measurements were performed on small aliquots of each sample using a Quantisorb Jr. surface area instrument (Quantichrome corporation, Greenvale, NY). This method of analysis uses a flow-through system in which a mixed helium/nitrogen gas is passed over the sample first at liquid nitrogen temperature and then at room temperature. The amounts of nitrogen adsorbed and desorbed during the cooling and heating cycle are used to calculate the surface area of the silica product (Gregg and sing, 1982).

\subsubsection{Initial Results}

The results of these tests, Table 3 , indicated that the silica 


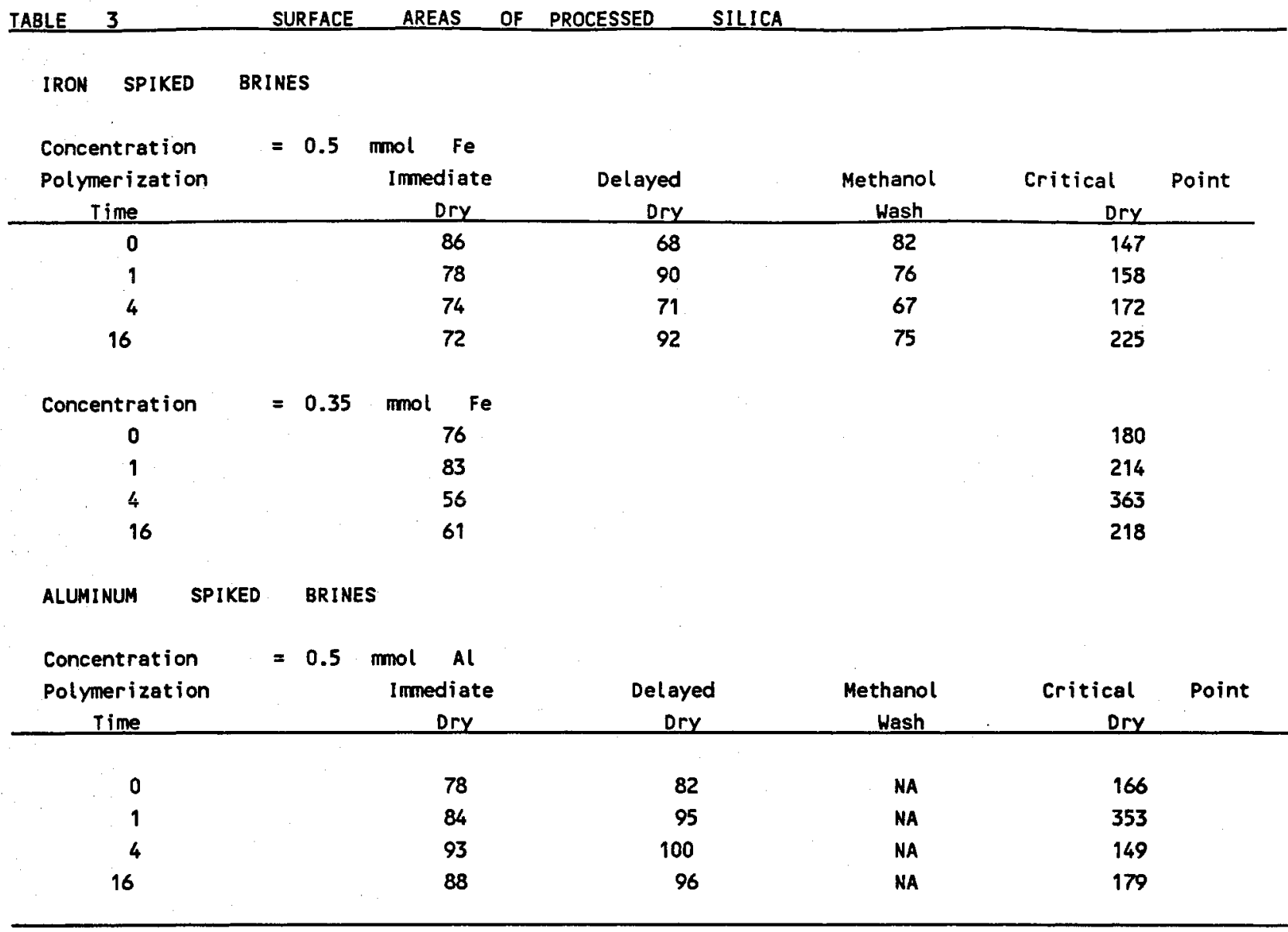

surface areas were typically in the range of 60 to $90 \mathrm{~m}^{2} / \mathrm{gm}$. The surface areas obtained with the aluminum spike were higher than those obtained with iron; however, no clear evidence of a trend was found between surface areas and polymerization time prior to spiking. In all cases, the surface areas were substantially below the levels required for a high-value silica product (150 $\left.\mathrm{m}^{2} / \mathrm{gm}\right)$.

\subsubsection{Additional Testing}

A further series of tests were conducted that varied the time between recovery and sample drying as well as the time between spike addition and pH adjustment; none of these attempts was successful in improving silica surface areas. Additional tests were conducted in which the water was removed from the gel by washing with methanol followed by oven drying; this procedure was also unsuccessful in increasing surface area. Finally, a series of 
tests were conducted that used supercritical carbon dioxide to remove the liquid phase from a methanol-washed silica product. The procedure developed for these tests was as follow:

a.) Gel samples were washed with distilled deionized water as described above;

b.) The gel was then washed with twice its volume of methanol in an ultrasonic bath for 5 minutes followed by centrifugation;

c.) The methanol treatment was repeated two more times;

d.) The methanol/gel sample was then loaded into a carbon dioxide critical-point dryer and dried with supercritical $\mathrm{CO}_{2}$ for a period of up to 40 minutes;

e.) The samples were sealed in an air-tight glass container until surface area measurements could be performed.

\section{2 .4 Results}

As is evident from Table 3 , the only significant improvement in surface area of the silica solid was found with critical point drying of the gel after a de-ionized water wash and subsequent removal of water with methanol. The surface areas of the solids obtained appear to be slightly higher when the brines were spiked with aluminum rather than iron but, in all cases, the surface areas were well above the minimum value of $150 \mathrm{~m}^{2} / \mathrm{g}$ required for specialty applications of the recovered silica.

A final set of runs was made to determine whether the concentration of the metal spike used had a significant impact on the surface area of the silica recovered. In this series, an iron spike was used with a final concentration of 0.35 mmolar. The results of this series shows that there is an apparent slight improvement in the average surface areas of the iron-recovered silica over that using a 0.5 molar concentration. Although a similar series of tests was scheduled to be performed using an aluminum spike at 0.35 molar, the termination of operations at the HGP-A well prevented this work from being completed.

The surface areas found with the critical-point dried samples also suggest that there may be a trend toward higher surface areas 
with polymerization times of from one to four hours; slightly lower areas were obtained for samples having longer or shorter times.

\section{2 .5 Analysis}

Our understanding of the mechanics of the polymerization process would tend to support this analysis:

a.) At very short polymerization times, the silica oligomers have time to form only very short chains; if these short chains are agglomerated by metal ion and caustic addition, the crosslinked units will be so small and so closely packed that gas molecules will be unable to penetrate the interparticle spacing and the effective surface areas will be relatively low:

b.) At intermediate polymerization times, the silica oligomers can form branched and cross-linked chains that are strong enough to be self supporting after the water has been removed from the network; hence, the dried, open network structure would be expected to have a substantial surface area;

c.) As polymerization times increase, the open network structure is progressively filled in by continued silica precipitation and polymerization; when this gel is dried, the more massive structure will be self supporting but will have a lower inherent surface area (Iler, 1979).

The differences between oven dried and critical-point dried silica surface areas show that post-precipitation treatment is critical to the ultimate surface areas obtainable. The reason for the large difference between the drying processes is that the silica network structures formed by the agglomerated silica gel are extremely fragile. When these networks are dried by normal means, the surface tension of the decreasing volume of the water film within the pores exerts stresses on the network that are capable of crushing the pore volume from within. Because critical point drying first fills the pores with a liquid and then, by heating and pressurizing the fluid, converts it to a gas phase without passing through a two-phase system, the solid network is not exposed to the crushing forces associated with the evaporative removal of a liquid phase. Hence, these fragile networks remain intact after the 
drying process. We found, however, that the silica solids were not completely stable with respect to repeated measurements of their surface area. If the samples were exposed to air, they showed a strong tendency to absorb moisture and subsequent surface area measurements showed decrease in specific surface area of as much as $20 \%$. Neither time nor resources allowed us to fully explore this phenomenon, but it is clear that the surface areas are highly dependent upon the post-recovery treatment of the silica gels.

\subsection{CONTAMINANT CONCENTRATIONS AND REMOVAL}

A second consideration in the value of the silica byproduct is its composition and the types and concentrations of contaminants co-precipitated with the silica. A series of experiments were performed that analyzed the solids for selected minor and trace elements and evaluated the effectiveness of acid washing the gels prior to drying.

\subsubsection{Experimental Procedure}

In this experiment silica slurries were recovered using both iron and aluminum treatments with a range of delay times as applied in the earlier experiment. The gel samples were washed with distilled deionized water (3X) and the gels were oven dried. Aliquots of each sample were dissolved in a 2:1 solution of hydrofluoric acid and aqua regia in a Parr bomb and the diluted solutions were analyzed for the selected elements. (Initial attempts to dissolve silica by open digestion resulted in loss of significant amounts of silica as $\mathrm{SiF}_{4} \cdot$ )

\section{3 .2 Results}

The silica analyses (Table 4) show that many of the dissolved ions present in the brine are retained by the silica. There appears to be very little consistent variation between the contaminant levels and the polymerization time prior to adding the metal ion spike for all elements, except calcium, which shows a slight decrease at longer times. Analyses of the supernatant for the iron series showed that suspended/dissolved silica in the fluid 
Alkali Metal Concentrations ( $\mathrm{mg} / \mathrm{kg}$ )

\begin{tabular}{lcccccccc} 
& $\mathrm{Br}$ & $\mathrm{Na}$ & $\mathrm{K}$ & $\mathrm{Rb}$ & $\mathrm{Mg}$ & $\mathrm{Ca}$ & $\mathrm{Sr}$ & $\mathrm{Ba}$ \\
\hline Brine & 37 & 5585 & 742 & 1.50 & 0.23 & 482 & 5.79 & 4.55 \\
Unspiked & 180 & 22330 & 4316 & 26 & 98 & 118160 & 330 & 741 \\
$4-\mathrm{Fe}-5.0$ & 450 & 54160 & 8549 & 34 & 144 & 84110 & 277 & 454 \\
4-Al-5.0 & 510 & 59940 & 8134 & 39 & 142 & 83040 & 268 & 444 \\
Pnd-85 & 60 & 7050 & 1494 & 7 & 68 & 34410 & 317 & 258
\end{tabular}

\begin{tabular}{|c|c|c|c|c|c|c|c|c|}
\hline & \multirow[b]{2}{*}{$\mathrm{Br}$} & \multirow[b]{2}{*}{$\mathrm{Na}$} & \multicolumn{2}{|c|}{ Enrichment } & Factor & \multirow[b]{2}{*}{$\mathrm{Ca}$} & \multirow[b]{2}{*}{$\mathrm{Sr}$} & \multirow[b]{2}{*}{$\mathrm{Ba}$} \\
\hline & & & $\underline{K}$ & $\mathrm{Rb}$ & $\mathrm{Mg}$ & & & \\
\hline Unspiked & 4.9 & 4.00 & 5.82 & 17.3 & 426 & 245 & 57.0 & 162.9 \\
\hline $4-\mathrm{Fe}-5.0$ & 12.2 & 9.70 & 11.52 & 22.7 & 626 & 175 & 47.8 & 99.8 \\
\hline $4-A L-5.0$ & 13.8 & 10.73 & 10.96 & 26.0 & 617 & 172 & 46.3 & 97.6 \\
\hline Pnd-85 & 1.6 & 1.26 & 2.01 & 4.7 & 296 & 71 & 54.7 & 56.7 \\
\hline
\end{tabular}
Transition Metal Concentrations
$(\mathrm{mg} / \mathrm{kg})$

\begin{tabular}{lrllllllr} 
& $\mathrm{Br}$ & $\mathrm{AS}$ & $\mathrm{Fe}$ & $\mathrm{Al}$ & $\mathrm{Mn}$ & $\mathrm{Zn}$ & $\mathrm{Cu}$ & $\mathrm{Cr}$ \\
\hline Brine & & & & & & & & \\
Unspik & 37 & 0.07 & 0.20 & 0.30 & 0.22 & 0.07 & 0.01 & 0.002 \\
4-Fe-5 & 180 & 5.5 & 35 & 340 & 390 & 30 & 10 & 0.6 \\
4-Al-5 & 450 & 3.6 & 870 & 240 & 230 & 22 & 10 & 4.1 \\
Pnd-85 & 510 & 0.5 & 560 & 79 & 230 & 28 & 15 & 5.6 \\
& 60 & 4.6 & 280 & 160 & 310 & 17 & $<5$ & $<0.5$
\end{tabular}

\begin{tabular}{|c|c|c|c|c|c|c|c|c|}
\hline & \multirow[b]{2}{*}{$\mathrm{Br}$} & \multirow[b]{2}{*}{ As } & \multirow{2}{*}{$\begin{array}{c}\text { Enrichme } \\
\mathrm{Fe}\end{array}$} & \multicolumn{2}{|c|}{ Factors } & \multirow[b]{2}{*}{$\mathrm{Zn}$} & \multirow[b]{2}{*}{$\mathrm{Cu}$} & \multirow[b]{2}{*}{$\mathrm{Cr}$} \\
\hline & & & & $\mathrm{Al}$ & $\mathrm{Mn}$ & & & \\
\hline Unspik & 4.9 & 81.8 & 174.6 & 1118 & 1773 & 461.5 & 857 & $30 c$ \\
\hline $4-\mathrm{Fe}-5$ & 12.2 & 53.5 & 4339.2 & 789 & 1045 & 338.5 & 857 & 2050 \\
\hline $4-A l-5$ & 13.8 & 7.4 & 2793.0 & 260 & 1045 & 430.8 & 1286 & 2800 \\
\hline Pnd -85 & 1.6 & 68.4 & 1396.5 & 526 & 1409 & 261.5 & - & 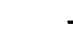 \\
\hline
\end{tabular}

phase after the "zero time" spike addition amounts to nearly fifteen percent of the total silica present whereas, after the one hour spike, the concentrations are one percent or less of the silica initially present. Hence, the increased efficiency of silica recovery accounts for the apparent decline in calcium concentration in the recovered solids.

\subsubsection{Analysis}

Although a complete analysis of the metal ion uptake by precipitating silica is beyond the scope of the present work, it 
is clear that several of the ion species present in the geothermal brine are highly enriched in the silica relative to some of the others. In a plot of the metal ion concentrations found (Fig. 12), given in terms of an Enrichment Factor (EF) (the concentration of the ion in the solid divided by its concentration in the brine) a number of trends are evident. Alkali ions show a clear tendency to be concentrated according to their charge characteristics: Sodium and potassium are the least retained in the solid whereas magnesium and calcium, which have a higher charge and charge density, are a factor of ten more highly enriched than are the alkalis. It is of note here that, in spite of the very divergent ion concentrations of the alkaline earth elements $(\sim 0.2 \mathrm{mg} / \mathrm{kg}$ for $\mathrm{Mg}$ to $\sim 450 \mathrm{mg} / \mathrm{kg}$ for $\mathrm{Ca})$, the enrichment factors are remarkably consistent with an affinity versus charge density model: The higher charge density of the positively charged magnesium ion shows a higher Enrichment Factor in the silica than does the calcium ion with a lower charge density. Although this relationship continues for the strontium ion, barium appears to break the trend of lower enrichment. Whether the latter ion interacts differently with the silica or whether the low solubility of $\mathrm{BaCO}_{2}$ forces its co-precipitation as a result of caustic addition is unknown at present.

The alkali and alkaline earth enrichments are also markedly different among the treatments used to recover silica. The samples denoted "Pond" were recovered from the fluid percolation ponds and were formed by natural precipitation of the silica. Those samples denoted "unspiked" were precipitated from the brine without the addition of metal ion to enhance precipitation; the "Fe" and "Al" samples were spiked with 0.5 millimolar iron and aluminum respectively. The naturally precipitated samples show the weakest enrichments of the four treatments with progressive enrichments through the un-spiked samples to nearly consistent enrichments for the metal ion spiked treatments. This progression is believed to result from the increased ion exchange 


\section{Alkali Metals Enrichment in Precipitated Silica}

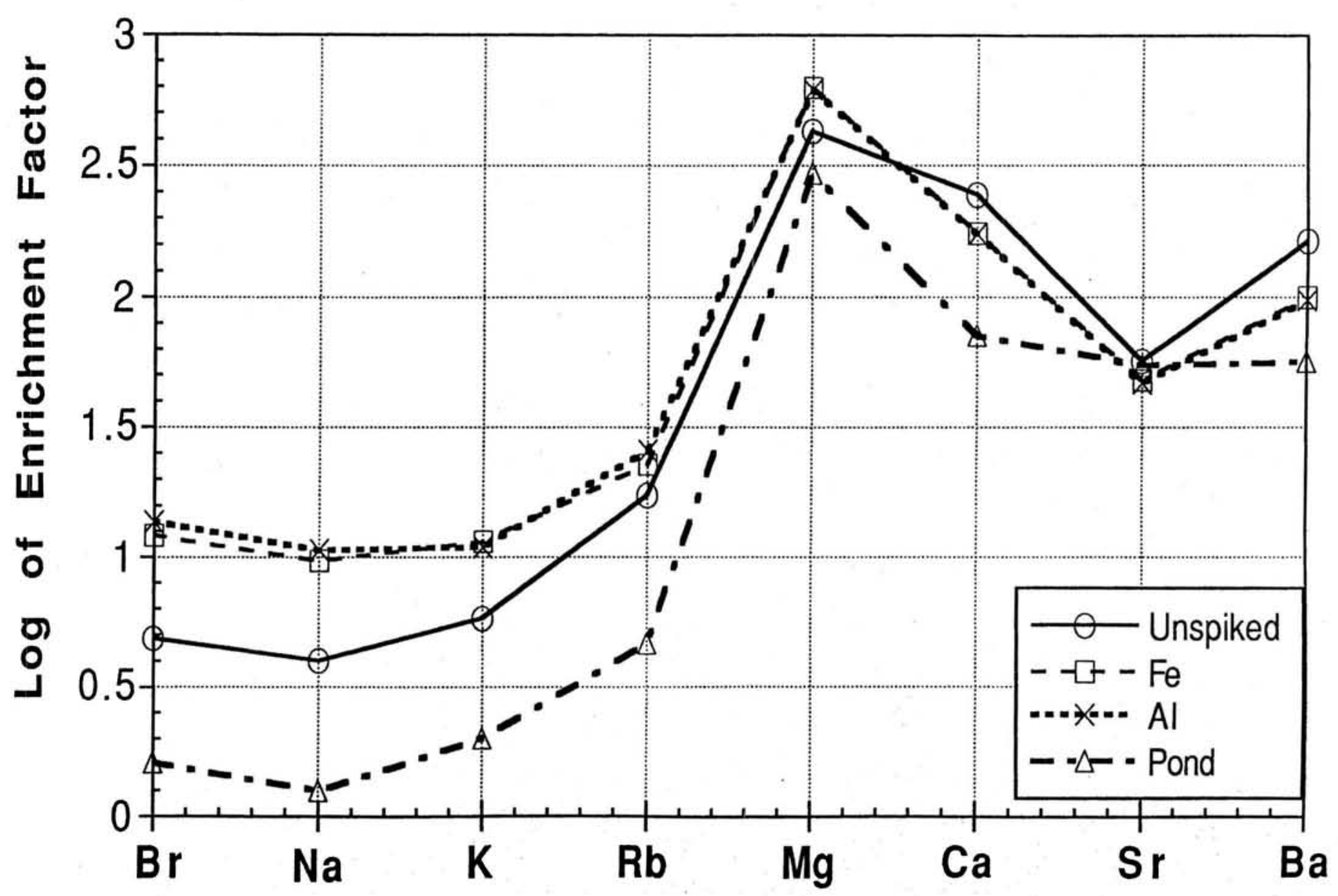

Figure 12. Alkali metals enrichment in silica precipitate from HGP-A geothermal fluids. 
capacity of the fluids at elevated pH (Allen and Matijevic, 1969; 1970, 1971; Allen et al., 1971; De Jong and Brown, 1980; Flemming and Crerar, 1982) as well as the increased volume and surface area of the gel. The naturally precipitated material typically has a higher density as a result of precipitation of silica monomer within the structure of the gel that gradually infills the open volume to the point where a dense vitreous solid is formed. Addition of caustic to the colloid substantially increases ionization of the surface silanols and ion exchange/ uptake during agglomeration of the silica polymers. The enhanced ion exchange results in a nearly four-fold increase in the sodium concentrations of the recovered solids. The increased ion concentrations within the spiked solids would suggest that the cross-linking by the metal ion addition further enhances the chemically active surface area of the agglomerated gel resulting in a still higher adsorption capacity of alkali ions.

A selected suite of transition metal ions were analyzed in the solids to determine how their levels and trends of enrichment in the solid compared with those of the alkali and alkaline earth ions. The analyses, conducted by a commercial neutron activation analysis laboratory (Activation Laboratories Ltd., Ontario, Canada, written communication), provided us with data for a wider range of metal ions with fewer sample preparation, and potential contamination, problems than would have been possible with traditional atomic absorption techniques. This method also provided data for bromine which, because it is not expected to participate in a chemical reaction with silica, shows what proportion of the salts are physically trapped rather than chemically bound to the silica matrix. Bromide ion concentrations (Fig. 13) show that interstitial water accounts for an enrichment of about a factor of ten in the dried solids recovered from the spike experiments and lesser amounts in the un-spiked and naturally precipitated solids. These results confirm our previous interpretation that sodium, potassium, and rubidium ions are very weakly bound within the silica matrix. 


\section{Trace Metal Enrichments in Precipitated Silica}

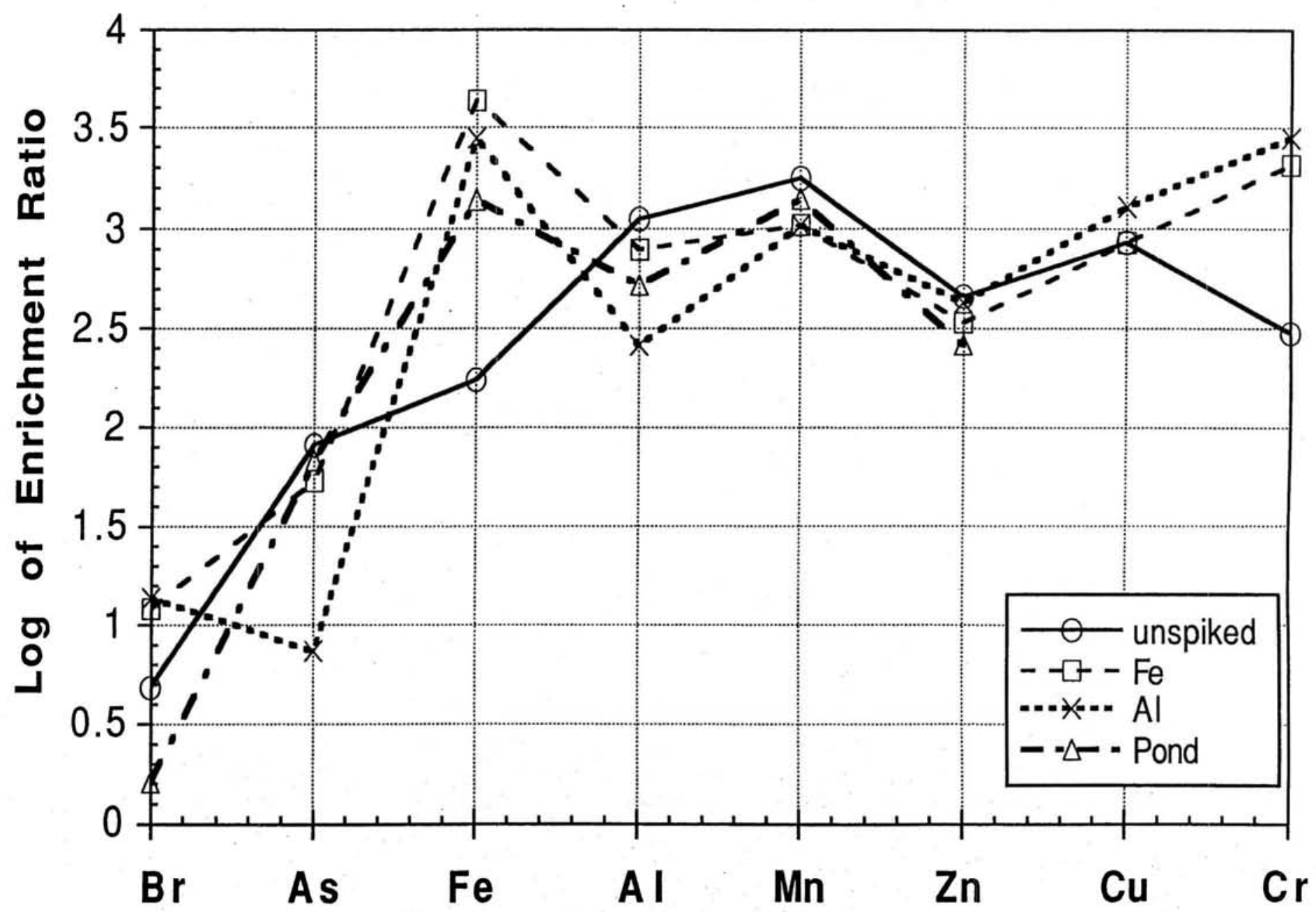

Figure 13. Trace transition metals enrichment in silica precipitate from HGP-A geothermal fluids. 
The transition metal ions show an enrichment significantly higher than that for the alkalis (Fig. 13). This is consistent with our earlier data and interpretation that the charge density of the highly charged metal ions render them extremely effective as charge neutralizers on the silica surfaces and to act as bridging points that increase the rate and extent of the polymerization process. The consistency of the Enrichment Factors of the transition metal ions suggests that differences in interaction between the silica and metal ions having similar charge density is relatively small. It is noteworthy that arsenic, although showing some enrichment in the silica over physical trapping, is clearly less enriched than the transition metals, which is consistent with its semi-metallic character.

\subsection{4 . Leaching Experiments}

As part of a companion investigation, a series of leaching experiments were performed to determine what acid concentrations are required to remove the physically and chemically adsorbed ions from the recovered silica (Decarlo, unpublished data). Weighed samples of dried silica were manually agitated with $20 \mathrm{ml}$ of dilute $\mathrm{HCl}$; the samples were centrifuged and washed; and the supernatant was analyzed for metal ion concentrations; the silica samples were then washed again with a higher concentration of $\mathrm{HCl}$ and the analytical process was repeated. The silica samples were sequentially treated with $\mathrm{HCl}$ concentrations of 0.05 $\mathrm{M}, 0.10 \mathrm{M}, 0.15 \mathrm{M}, 0.20 \mathrm{M}, 0.35 \mathrm{M}, 0.50 \mathrm{M}$, and $2.0 \mathrm{M}$. The results of these treatments, presented as cumulative percentage removals (Fig. 14, 15, and 16), show that acid washing with dilute hydrochloric acid is capable of removing a substantial proportion of the sodium and potassium from the solids. Although magnesium appears to be very tightly bound, with typically less than $50 \%$ to $60 \%$ being removed with 2 molar hydrochloric acid, calcium is more easily lost. The response of the iron and aluminum to acid treatment shows that they are removed in an inverse proportion to their concentration: If iron was used as a spike to precipitate the silica, more than $60 \%$ of it could be 


\section{Acid Leaching of $\mathrm{Fe}_{2}\left(\mathrm{SO}_{4}\right)_{3}$ Catalyzed Silica Precipitate}

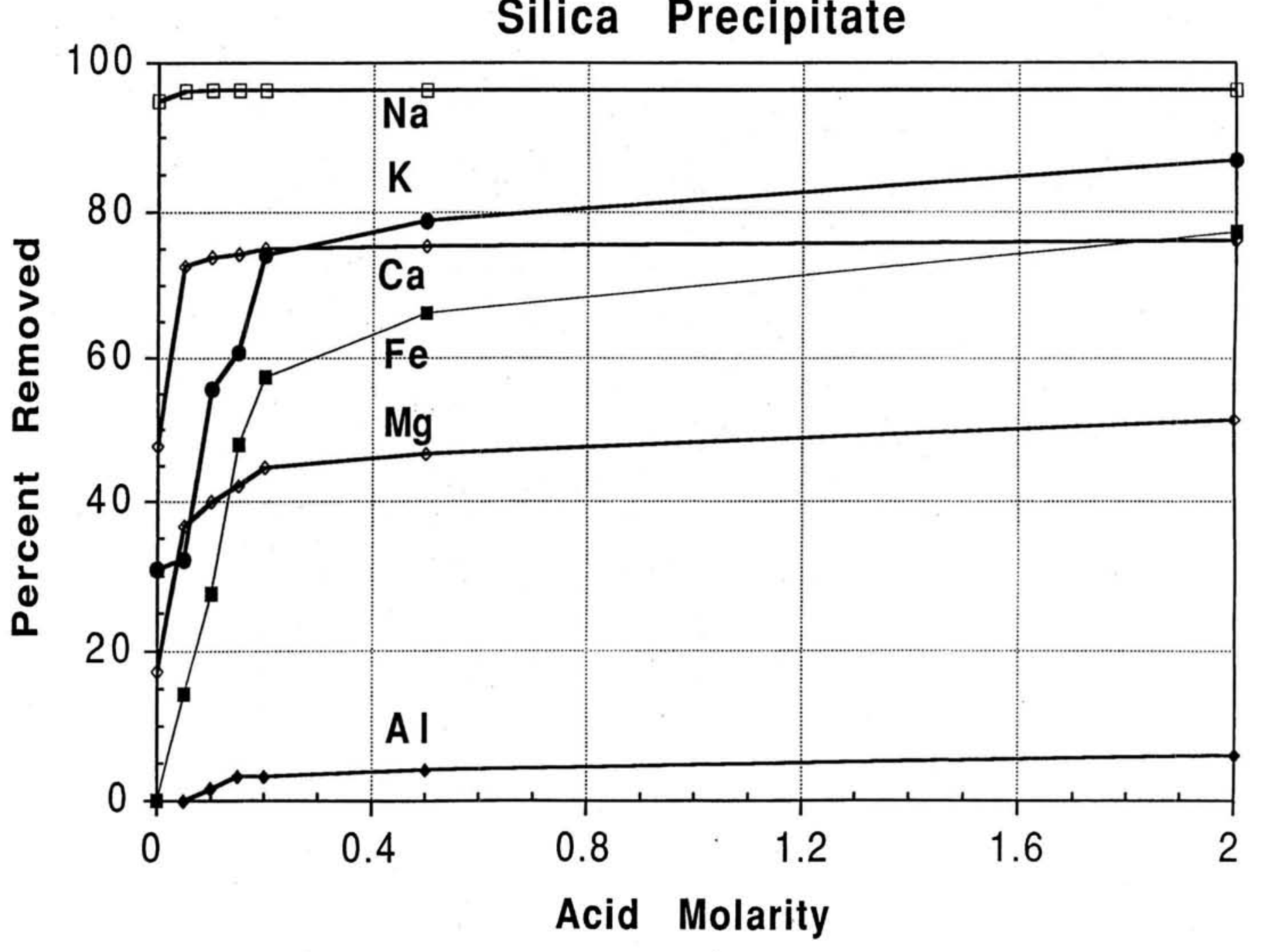

Figure 14. Acid leaching of alkali and transition metals from silica precipitated from geothermal fluid by iron addition. 


\section{Acid Leaching of $\mathrm{Al}_{2} \mathrm{SO}_{4}$ Catalyzed Silica Precipitate}

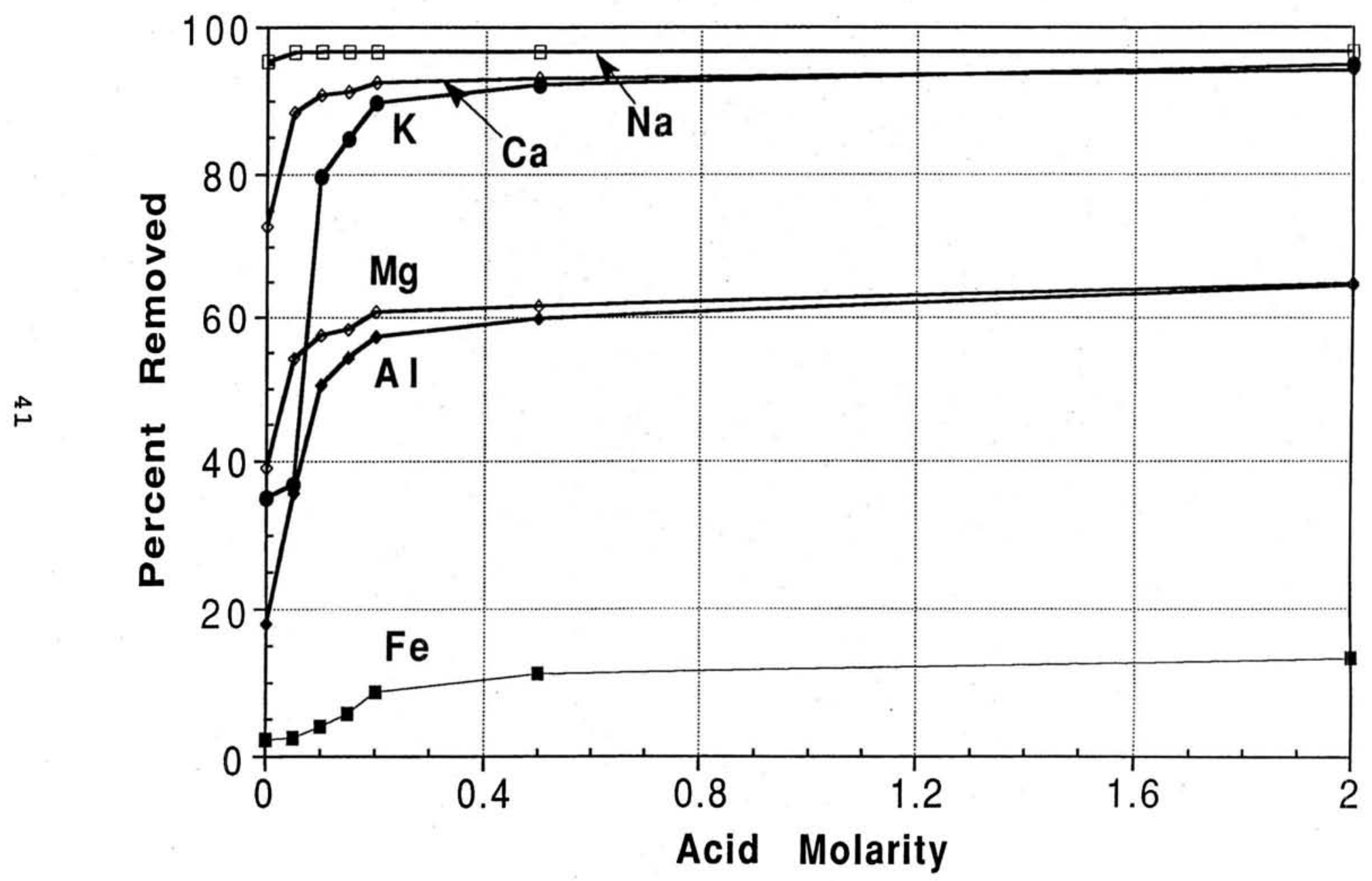

Figure 15. Acid leaching of alkali and transition metals from silica precipitated from geothermal fluid by alluminum sulfate addition. 


\section{Acid Leaching of $\mathrm{KAI}\left(\mathrm{SO}_{4}\right)_{2}$ Catalyzed Silica Precipitate}

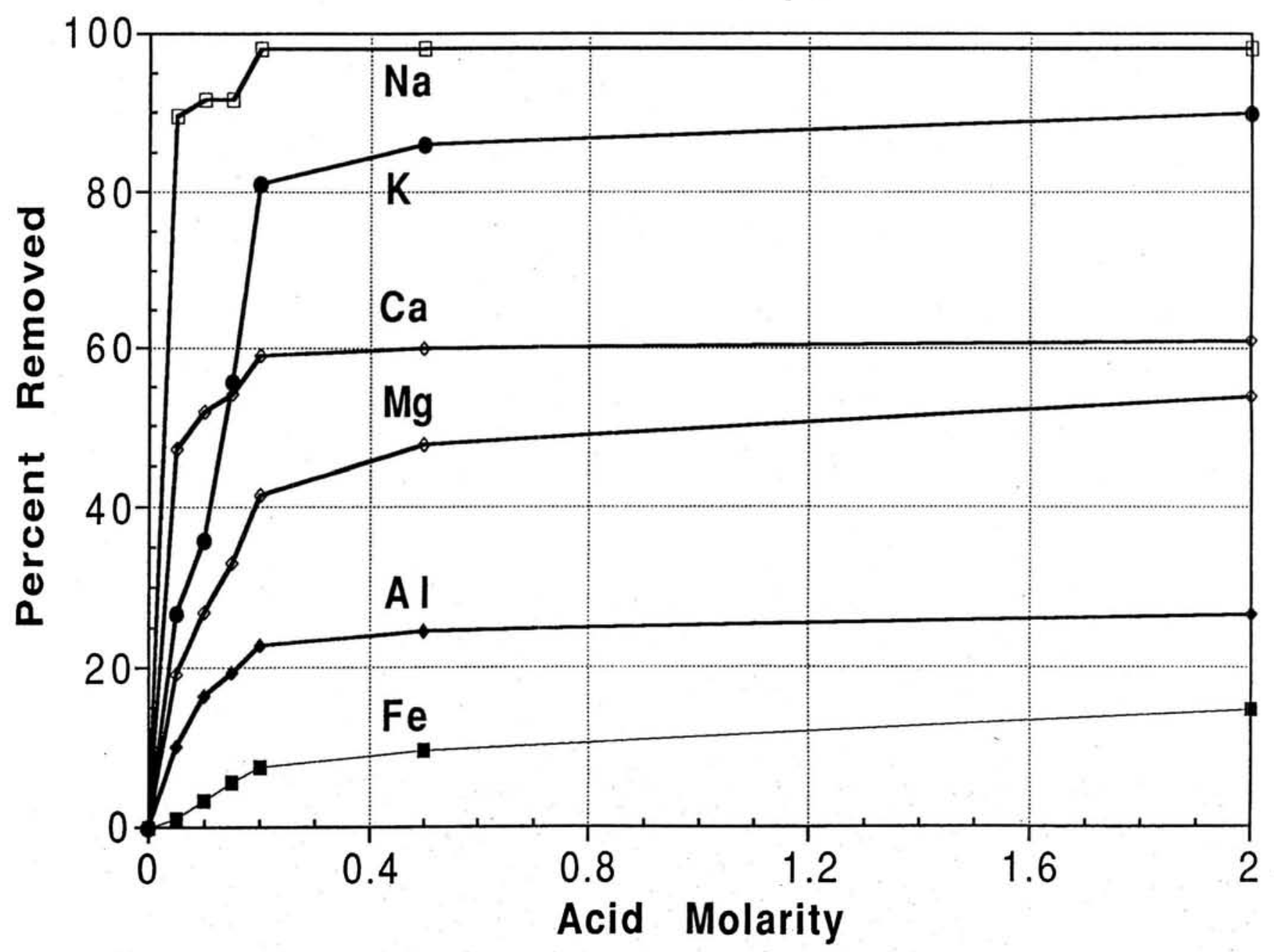

Figure 16. Acid leaching of alkali and transition metals from silica precipitated from geothermal fluid by potassium alluminum sulfate addition. 
removed with acid concentrations of less than $1 M$ whereas less than $5 \%$ of the aluminum present in this same sample was removed at $2 \mathrm{M}$ concentration of $\mathrm{HCl}$. The pattern is reversed when aluminum is used as the precipitating agent: $60 \%$ of the aluminum is removed with $1 \mathrm{M} \mathrm{HCl}$ but only about $15 \%$ of the iron is removed at $2 \mathrm{M}$. Although this response is not unexpected, it does demonstrate that only a small amount of the metal ion is chemically bound in the structure of the silica after it is agglomerated. It also indicates that the metal ion spike can be effectively removed and the silica solids cleared of most contaminant ions with a mild acid treatment.

\subsection{SUMMARY AND CONCLUSIONS}

These investigations have demonstrated that optimum silica recoveries could be achieved using $0.5 \mathrm{millimolar}$ additions of aluminum or iron to the geothermal fluids in order to enhance the polymerization process and that the addition of caustic to a pH range of 10.5 to 11.5 achieved maximum flocculation rates. slower rates of flocculation and precipitation were observed at lower pH levels. Whether a reduction in caustic usage may make lower $\mathrm{pH}$ recoveries more economically viable on a commercial scale than those at which maximum precipitation occurred would, however, have to be determined through an economic analysis of a recovery system.

Optimization studies have shown that the surface area of the recovered material can be substantially increased by using a supercritical drying process to remove trapped water from the recovered silica gels. The surface area of the final product can also be increased by polymerizing the dissolved silica in the brine prior to the addition of precipitating or flocculating agents. The silica recovery procedure developed in the present work produced silica with surface areas of more than $250 \mathrm{~m}^{2} / \mathrm{gm}$ which easily exceeded the minimum surface areas of $150 \mathrm{~m}^{2} / \mathrm{gm}$ that were considered necessary for a high value silica product. 
Chemical analyses of the solids showed that some of the dissolved species present in the brine phase were enriched in the silica by factors of ten or more over their concentrations in the liquid phase. The alkali ions were enriched by a factor of ten in the solids and were easily removed with a dilute $\mathrm{HCl}$ wash. of the alkaline earths, calcium was the most easily removed. Magnesium and the transition metals showed substantially greater resistance to removal with dilute acid suggesting that they are taken up in the structure of the silica solids.

These studies indicate that it is technically feasible to reduce the silica concentration in Hawaii's geothermal brines to levels well below those required to prevent precipitation of quartz or amorphous silica at surface conditions. It has also been demonstrated that it is technically feasible to recover and process the silica in a manner that will yield a saleable byproduct.

Because of the premature shut-down of the operation of the HGP-A geothermal facility, we were unable to demonstrate a continuous silica recovery process at the pilot scale. Although a continuous treatment system was designed and fabricated, as described in the following, inadequate time was available to conduct reagent injection and silica recovery tests. Such testing is needed in order to determine the economic feasibility of the batch processes developed during the present effort. 


\section{SILICA INHIBITION STUDIES}

\subsection{OBJECTIVES}

Another goal of our silica control investigations was to develop a method of inhibiting silica precipitation from the geothermal brines. This effort was intended to protect surface equipment and to permit reinjection of dissolved and colloidal silica back into the subsurface formation along with other waste geothermal fluids. The results of our batch studies, as well as those of other researchers in the field (Iler, 1979; Rothbaum et al., 1979, Weres et al., 1980; Weres and Tsao, 1981), demonstrated that acidification of the geothermal fluids tended to inhibit silica flocculation or precipitation from the fluid phase. Acidification of the geothermal reinjectate with common industrial acids (e.g. $\mathrm{H}_{2} \mathrm{SO}_{4}$ or $\mathrm{HCl}$ ) could clearly accomplish this task. However, consideration of the increased costs as well as the potential for increasing silica polymerization at low pH levels (2 to 3) (Iler, 1979) suggest that a weakly acid or moderate $\mathrm{pH}$ buffering agent may be a more prudent approach. An obvious choice for a weakly acid reagent with which to lower the pH of the reinjectate fluids is the non-condensable gas (NCG) stream derived from the geothermal steam: both of the major components of the NCG stream, carbon dioxide and hydrogen sulfide, are weak acids, they are typically present at high enough concentrations in most geothermal fluids to lower the reinjectate $\mathrm{pH}$ into the range of silica stability ( $\mathrm{pH} 3$ to $\mathrm{pH} 6)$, and they frequentiy must be abated or disposed of regardless of whether they are used in this process.

\subsection{TEST STAND DESIGN}

In order to test the effectiveness of recombining noncondensable gases (NCG) with the geothermal fluids, a test system was designed and fabricated that could be used to mix the NCG, as well as other reagents, into a side-stream of hot, pressurized, 
geothermal fluids from the HGP-A plant separator. The system was designed to permit an analysis of: The silica response to a reduction in reinjectate $\mathrm{pH}$; The impact of the addition of NCG on the stability of other components of the geothermal fluid (e.g. metal sulfides); and The potential corrosion effects of the NCG on metal alloys typically available for use in a geothermal field.

The specific design criteria used in the fabrication of the test stand were:

a.) Continuous flow capacity of the combined fluid streams of one cubic meter per hour (16.7 1/min; $4.4 \mathrm{gpm})$;

b.) Mixing of brine, condensate, and non-condensable gas streams at a range of proportions and at a range of temperatures;

c.) Direct observation of the effects of fluid recombination on the solids load present in the brine phase;

d.) Direct sampling of the mixed phases;

e.) Exposure of metallurgical and rock samples to the mixed phase fluids.

A line diagram of the test system is presented in Figure 17. It was comprised of four basic units: the brine, condensate, and non-condensable gas/reagent inlet systems, and the mixed phase test system. The brine inlet consisted of an insulated supply line, operated at the separator discharge pressure (nominally 11.4 bar (165 psia)) and temperature (nominally $187^{\circ} \mathrm{C}\left(368^{\circ} \mathrm{F}\right)$ ), a single pass, tube-in-tube, heat exchanger (COOLER, Fig. 17), and a check valve that fed brine into the test system through a flow control valve. Temperature and pressure were monitored on this line but, because of the high temperature and high potential for scaling, neither flow metering nor $\mathrm{pH}$ measuring devices could be exposed to the brine.

The condensate inlet accepted fluid from the condenser discharge $\left(T=51^{\circ} \mathrm{C}\left(124^{\circ} \mathrm{F}\right) ; \mathrm{P}=2.4\right.$ bar $(35 \mathrm{psia}) ;$ a positive displacement pump pressurized the condensate to the system operating pressure after which it passed through a single-pass heat exchanger, a flow control valve, and check and flow control valves 


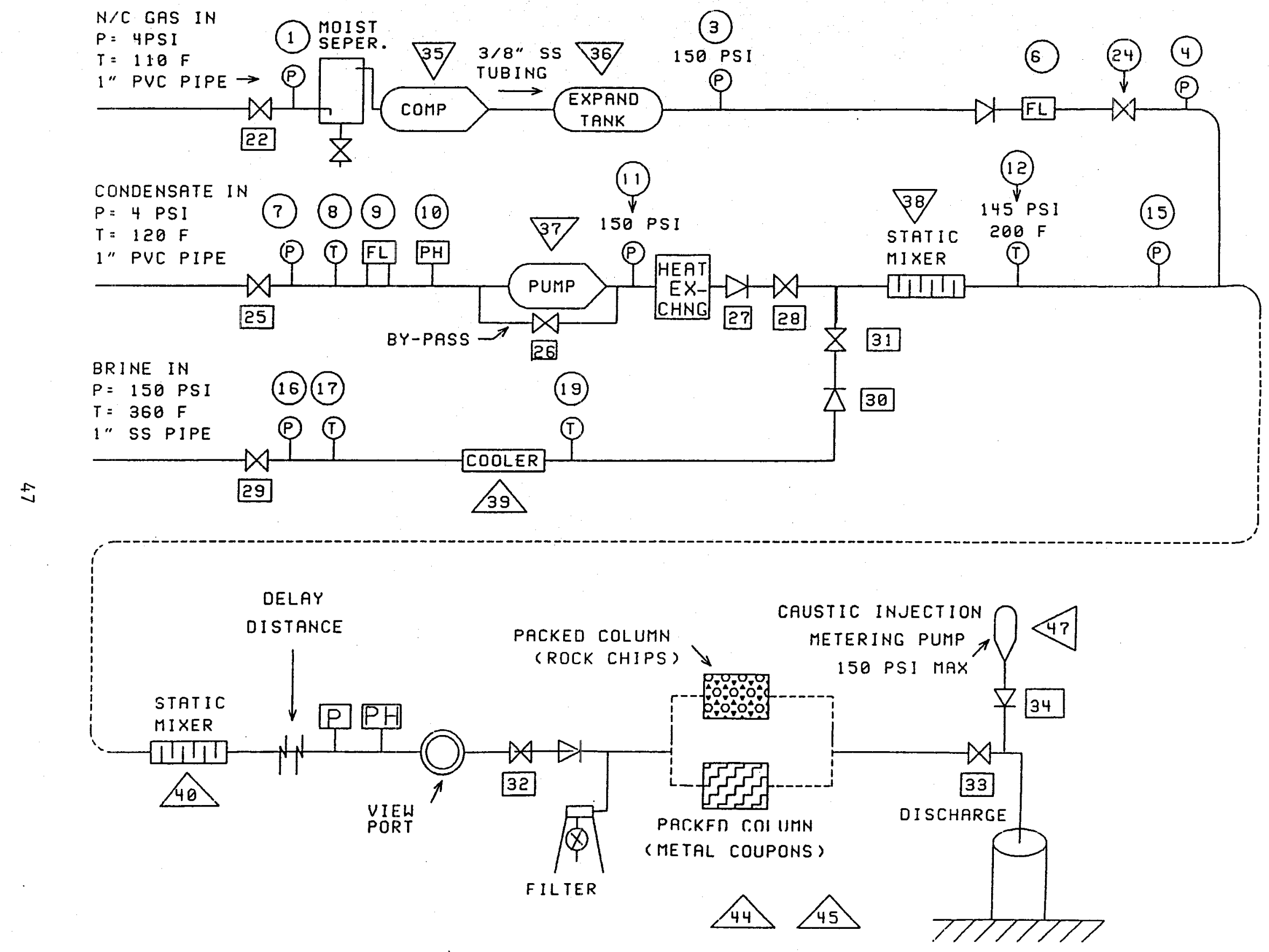

Figure 17. Line diagram of the bench-scale test system fabricated for mixing geothermal brine with silica precipitating and silica inhibiting 
that injected condensate into the system downstream of the brine inlet. Condensate injection pressures were controlled using a pressure relief by-pass line around the positive displacement pump. The lower temperatures and pressures in the condensate line permitted continuous monitoring of the fluid pH and flow rate as well as its temperature and pressure.

The non-condensable gas line is supplied by the condenser offgas line $\left(T=38^{\circ} \mathrm{C}\left(100^{\circ} \mathrm{F}\right) ; \mathrm{P}=1.3\right.$ bar $\left.(19 \mathrm{psia})\right)$, through a moisture separator, and compressor. The compressed gas is passed into a ballast tank and through a check valve and flow control valve into the mixed-phase line. Pressure and flow rate were monitored on the gas line immediately prior to injection into the mixed-phase line. The gas injection port was also designed for use as the injection point for other reagents that were to be mixed into the brine/condensate stream during the subsequent testing that was forestalled by the shut-down of the generator facility.

The mixed-phase system was designed to accept and mix the liquid and gas from the three inlet lines and to permit testing of the characteristics of the mixed phase fluid. The line consisted of a static mixer, immediately downstream of the brine and condensate injection points, followed by temperature, pressure, $\mathrm{pH}$, and flow measuring devices. The gas/reagent injection port entered the system downstream of the liquid mixing and monitoring equipment, and was followed by a second static mixer, a variable length "delay-line," a view port, a testing manifold and a discharge line. The functions of each element of the mixed-phase line were:

Static mixers: ensure proper mixing of the liquid components of the test stream and generate adequate contact area between the gas and the liquid phases in the line;

Delay line: evaluate possible kinetic effects controlling the reaction of the fluids with the injected gases or reagents by extending or decreasing the contact time between fluids and reagents; 
View Port: permit qualitative observations of solids precipitation and the degree of gas dissolution;

Testing manifold: permit fluid sampling and installation of exposure chambers to test for corrosion and scaling potential of the mixed phase fluids;

Discharge system: carry fluids to the plant discharge system and for treatment of the non-condensable gases with cooling water and hydrogen sulfide abatement chemicals.

In order to minimize contamination of the geothermal fluids with corrosion products from the test system, the mixed phase line was fabricated of type 316 stainless steel and stainless steel reinforced teflon. The condensate and the non-condensable gas feed lines were fabricated of PVC pipe, the view port was of heavy-walled glass tubing, and the non-condensable moisture separator and gas compressor were fabricated of mild steel. All liquid flow lines were 1 -inch (inside) diameter pipe except for the delay coils which were $3 / 4$ inch stainless steel reinforced teflon flexible hose. Heat loss from the fluid was reduced by insulating all high temperature lines with 1 -inch thick fiber glass.

\subsection{NON-CONDENSABLE GAS INJECTION TEST PLAN}

This series of tests evaluated the effectiveness of adding weakly acidic non-condensable gases to the geothermal reinjectate as a means of inhibiting silica flocculation and precipitation. The experiment was also designed to determine whether sulfide deposition or increased corrosion was likely to result from this method of silica control. Because the results of the study were of direct significance to the design of commercial geothermal facilities in Hawaii, our experimental approach was to first evaluate the dissolved silica response to the addition of NCG to a mixed fluid having a chemical composition similar to a reinjectate derived from the nearby private geothermal well KS1-A, located on the Puna Geothermal Venture lease. Our test plan was to complete the testing of the KSI-A reinjectate and then to continue testing the silica response to the addition of gas, and other reagents, 
over a broad range of reinjectate compositions. The unanticipated termination of operations at the HGP-A generator facility, however, forced us to abbreviate the planned gas injection testing and precluded our analysis of the effects of other reagents on silica deposition under flowing conditions.

The design parameters for a planned commercial power plant using KS1-A fluids were used to calculate a reinjectate composition containing a silica concentration of about $340 \mathrm{mg} / \mathrm{kg}$ and a hydrogen sulfide concentration of $1000 \mathrm{mg} / \mathrm{kg}$ at a temperature of $100^{\circ} \mathrm{C}$ $\left(212^{\circ} \mathrm{F}\right)$. Although formulation of a reinjectate fluid having a chemical composition identical to KS1-A was not considered to be technically feasible, we were able to mix brine, condensate, and non-condensable gas from HGP-A in the test system to produce a fluid with key parameters similar to those of reinjectate expected from the commercial facility (Table 5). Because the dissolved solids concentrations in the KSI-A brines were higher, and the proportion of brine produced was greater, than in any of the other wells drilled on the Puna Geothermal venture lease up to that time, the KS1-A reinjectate was considered a "worst case" situation for fluid disposal.

Evaluation of the physical and chemical response of the fluids to mixing included assessment of variations in $\mathrm{pH}$ of the mixed phases; extent of homogeneous (fluid phase) precipitation of silicates and sulfides; extent of heterogeneous precipitation of silicates and sulfides onto metal and basaltic substrates; rate and degree of dissolution of the mixed gas into the reinjectate liquid phase; and corrosivity of the mixed phases on various alloys of stainless and mild steel.

The test stand was instrumented with high pressure and temperature $\mathrm{pH}$ probes that monitored fluid $\mathrm{pH}$ downstream of the liquid and gas phase injection and mixing points. Samples of fluid were obtained through a filtration device that permitted a determination of the extent of homogeneous precipitation of sulfides. Samples of rock chips and corrosion coupons were exposed 


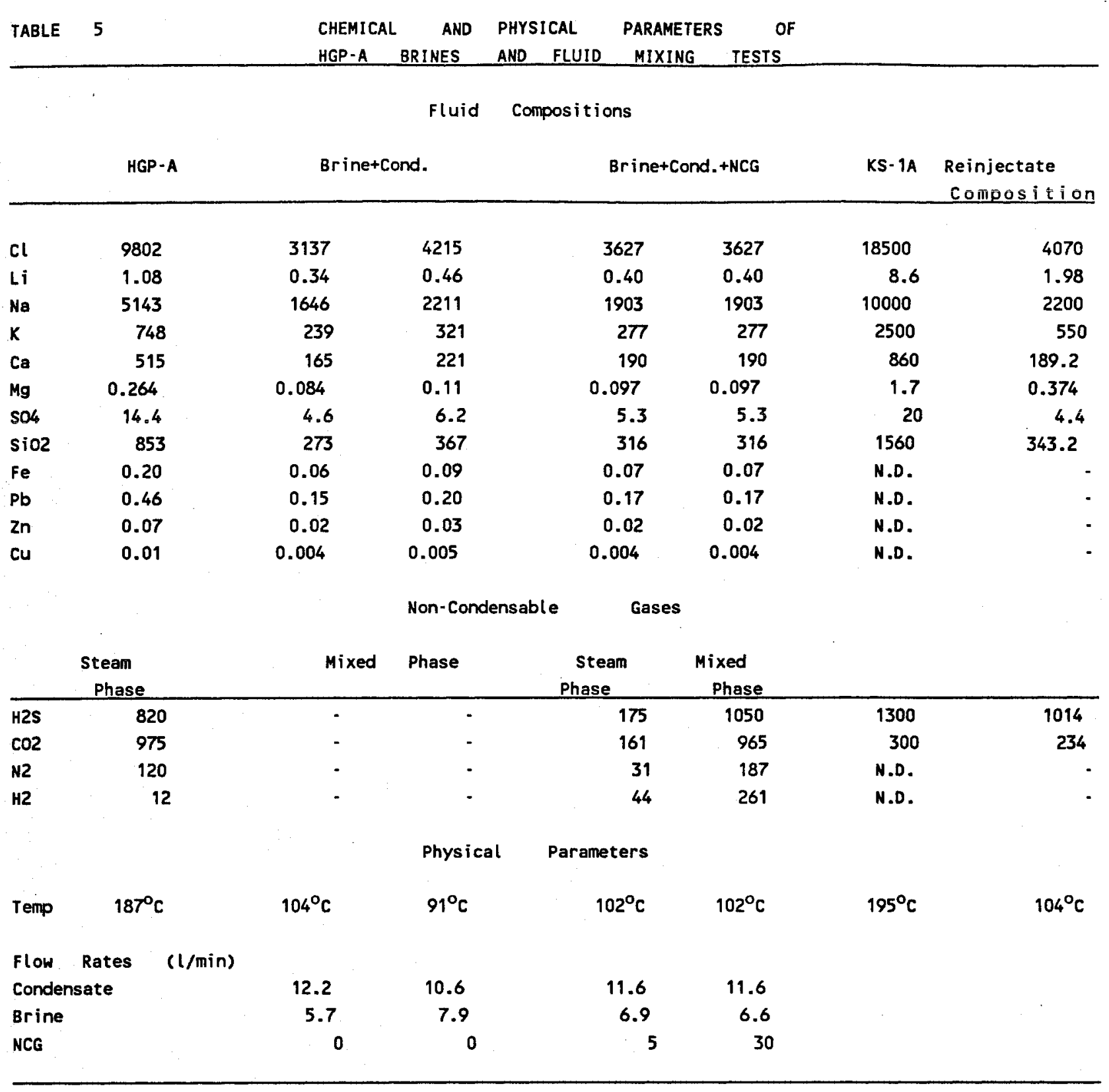


to the mixed phase fluids in a pair of test cylinders that provided data on both the rates of heterogeneous deposition of precipitates and the corrosivity of the mixed fluids. The delay coil, consisting of a set of flexible woven stainless steel-reinforced teflon lines, could be connected together enabling the fluids to pass through lengths of tubing ranging from $4 \mathrm{~m}$ to $35 \mathrm{~m}$ and extend the contact time of the gas and liquid from about $5 \mathrm{sec}$. to $45 \mathrm{sec}$. Qualitative observations were made of the mixed phase downstream of the various lengths of delay coil in an effort to make gross determinations of variations in gas dissolution with changes in contact time.

\subsection{EXPERIMENTAL TRIALS PERFORMED}

Two experimental trials were conducted: evaluation of the impacts of mixing the brine and steam condensate streams and analysis of the response to mixing all three streams -- brine, condensate, and non-condensable gases -- together. Although additional experiments were planned, termination of operations at the HGP-A Generator facility precluded the evaluation of a broader range of reagents, fluid compositions, and temperatures.

4.4.1 Brine/Condensate Mixing

The brine/condensate test was conducted as follows (see Appendix 2 for a detailed discussion):

a.) The system was prepared by loading and installing the exposure tanks. One tank was loaded with two coupons each of SS-316L and SAF-2205 steel: one sample of each alloy was installed in a stressed state and the other in an unstressed state. The other tank contained washed and sieved 3-mm basalt chips.

b.) The system was pressurized with steam condensate to its operating pressure of 15 bars at a flow rate of about $11 \mathrm{l} / \mathrm{min}$. After temperature and flow equilibrium were achieved with condensate alone, brine was admitted into the system and the flows were balanced until the appropriate temperature and silica concentration were achieved in the mixed phase. During normal 


\begin{tabular}{|c|c|c|c|}
\hline Date & $\begin{array}{r}\text { Height } \\
\text { Change } \\
\text { (Milligrams) } \\
\end{array}$ & $\begin{array}{r}\text { Liquid } \\
\text { Volume } \\
\text { (Liters) }\end{array}$ & $\begin{array}{l}\text { Ppt. Mass } \\
\text { Per Liter } \\
(\mathrm{mg} / \mathrm{l}) \\
\end{array}$ \\
\hline $11 / 26 / 89$ & 10.7 & 77 & 0.139 \\
\hline $11 / 28 / 89$ & 35.2 & ND & - \\
\hline $12 / 2 / 89$ & 4.21 & 200 & 0.2105 \\
\hline $12 / 3 / 89$ & 35.2 & 180 & 0.196 \\
\hline $12 / 3 / 89 *$ & 1.7 & 20 & 0.085 \\
\hline $12 / 5 / 89$ & 17.4 & 240 & 0.0725 \\
\hline $12 / 8 / 89$ & 76.9 & 100 & 0.769 \\
\hline * 24 hour & nucleation & & \\
\hline
\end{tabular}

Additional fluid samples were also stored in closed glass containers for qualitative observation of colloid formation over time. These samples showed minimal turbidity for several weeks and only gradually began developing a bluish cast after more than a month of polymerization time. By comparison, raw brine from HGP-A showed strong turbidity almost immediately after flashing and a gelatinous deposit after settling periods of several hours at ambient temperatures (Plate 3 ).

\subsubsection{Corrosion and Heterogeneous Precipitation}

The $\mathrm{pH}$ of the mixed fluids was slightly acid and ranged from 5.7 to 6.3 depending on the proportion of brine to condensate. These values suggest that the buffering capacity of the brine is adequate to tolerate the injection of substantial amounts of condensate without having the $\mathrm{pH}$ fall to levels that are at equilibrium with carbonic acid present in the condensate ( $\mathrm{pH} 4.5$ ).

Microscopic inspection of the corrosion coupons exposed to the mixed fluids found no detectable scale deposition or corrosion resulting from exposure to the fluids. The sample weights after 


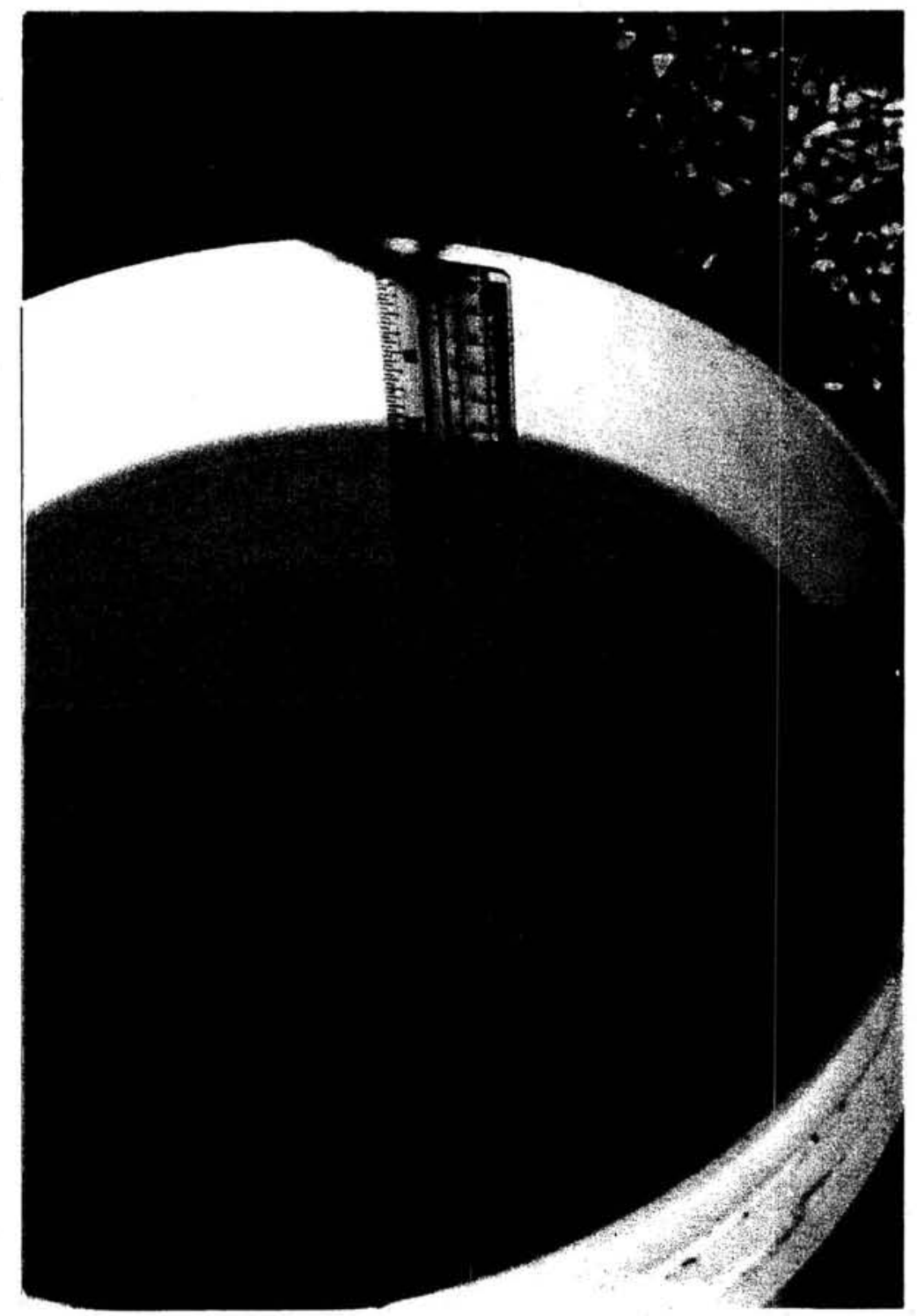

Plate 1. Mixed geothermal brine and steamcondensate showing absence of suspended particulates immediately after mixing and filtration.

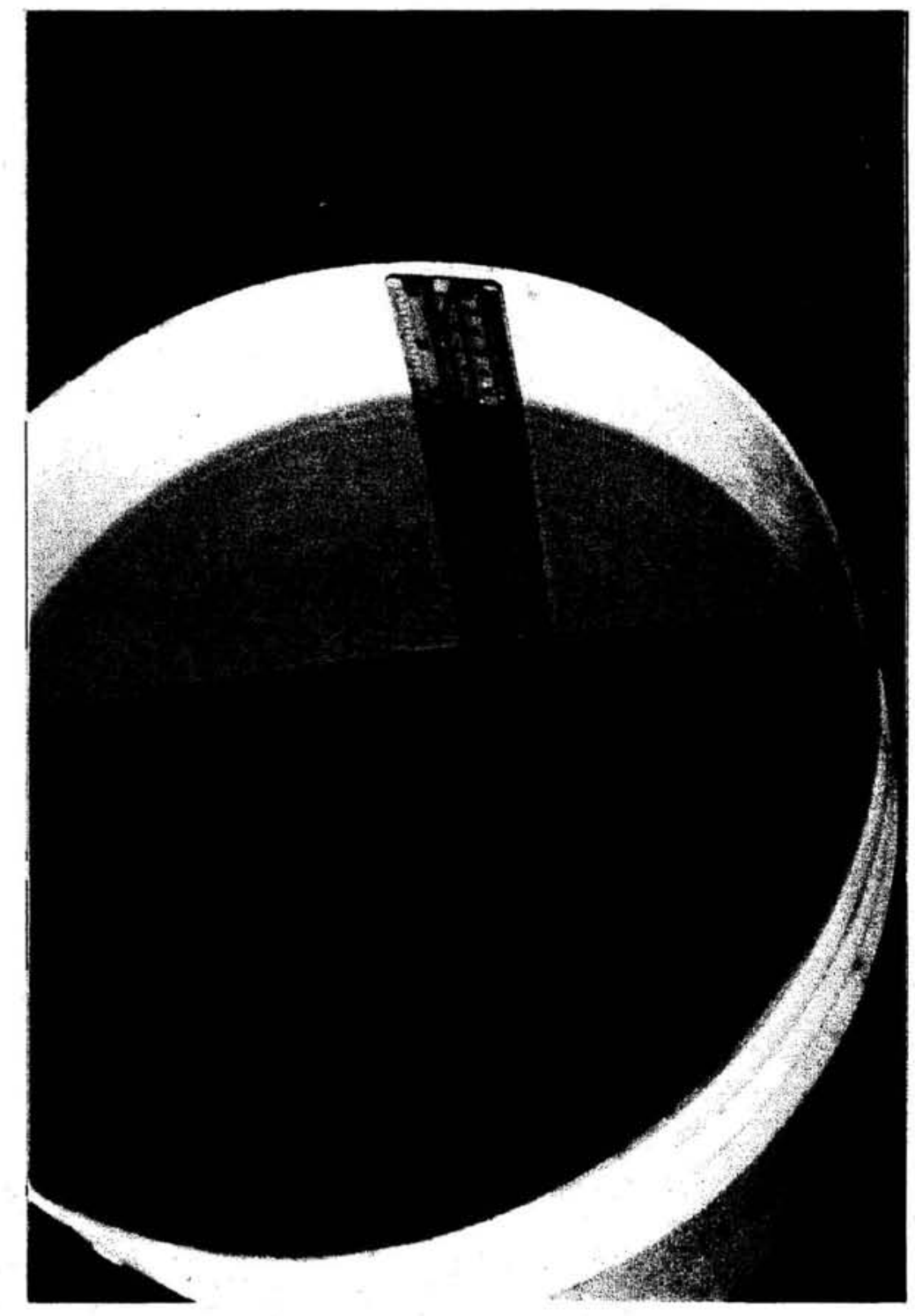

Plate 2. Mixed geothermal brine and steam condensate, as in Plate 1, after 24 hours of polymerization at ambient temperature. 


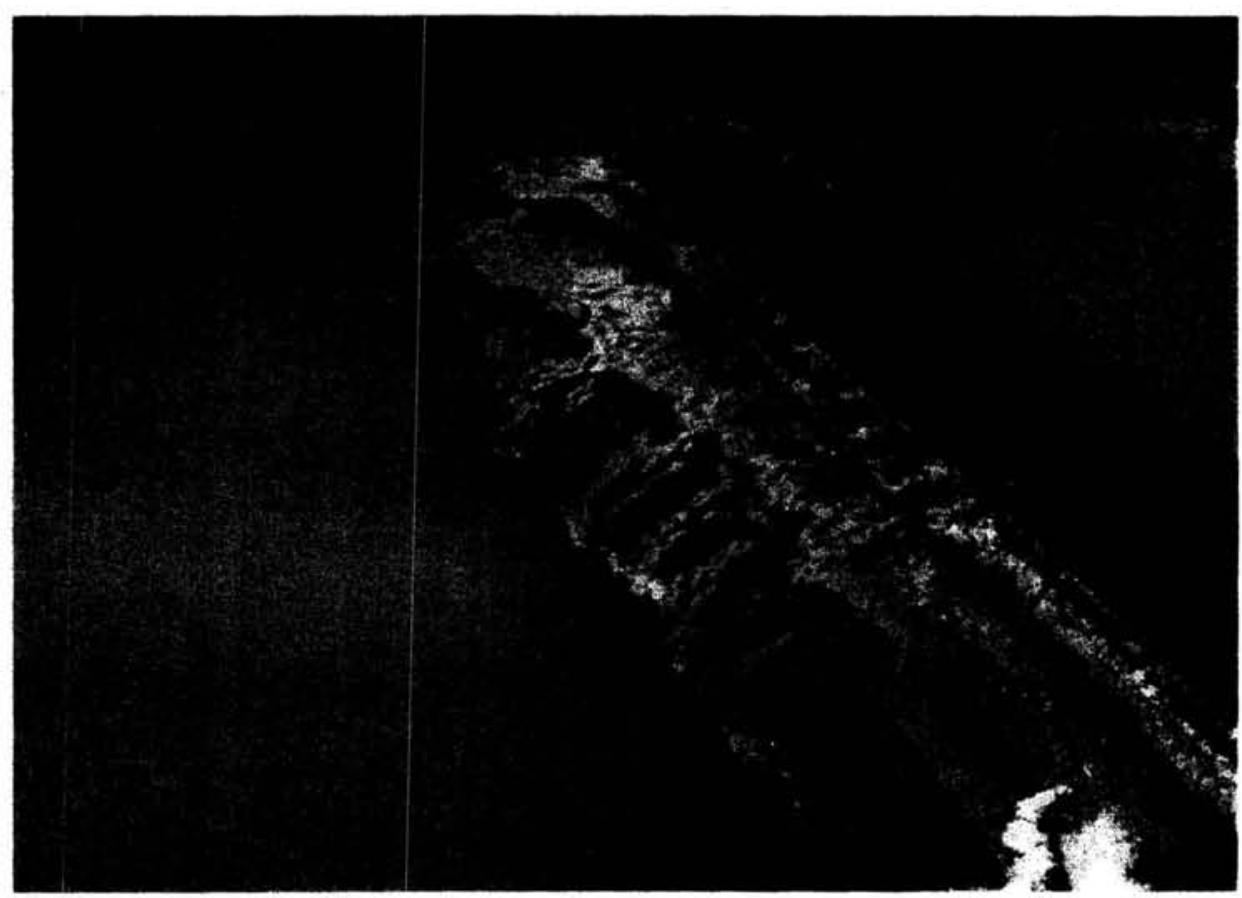

Plate 3. Pure geothermal brine immediately after flashing to atmospheric pressure and $100^{\circ} \mathrm{C}$ showing high concentration of colloidal suspended particulates. 


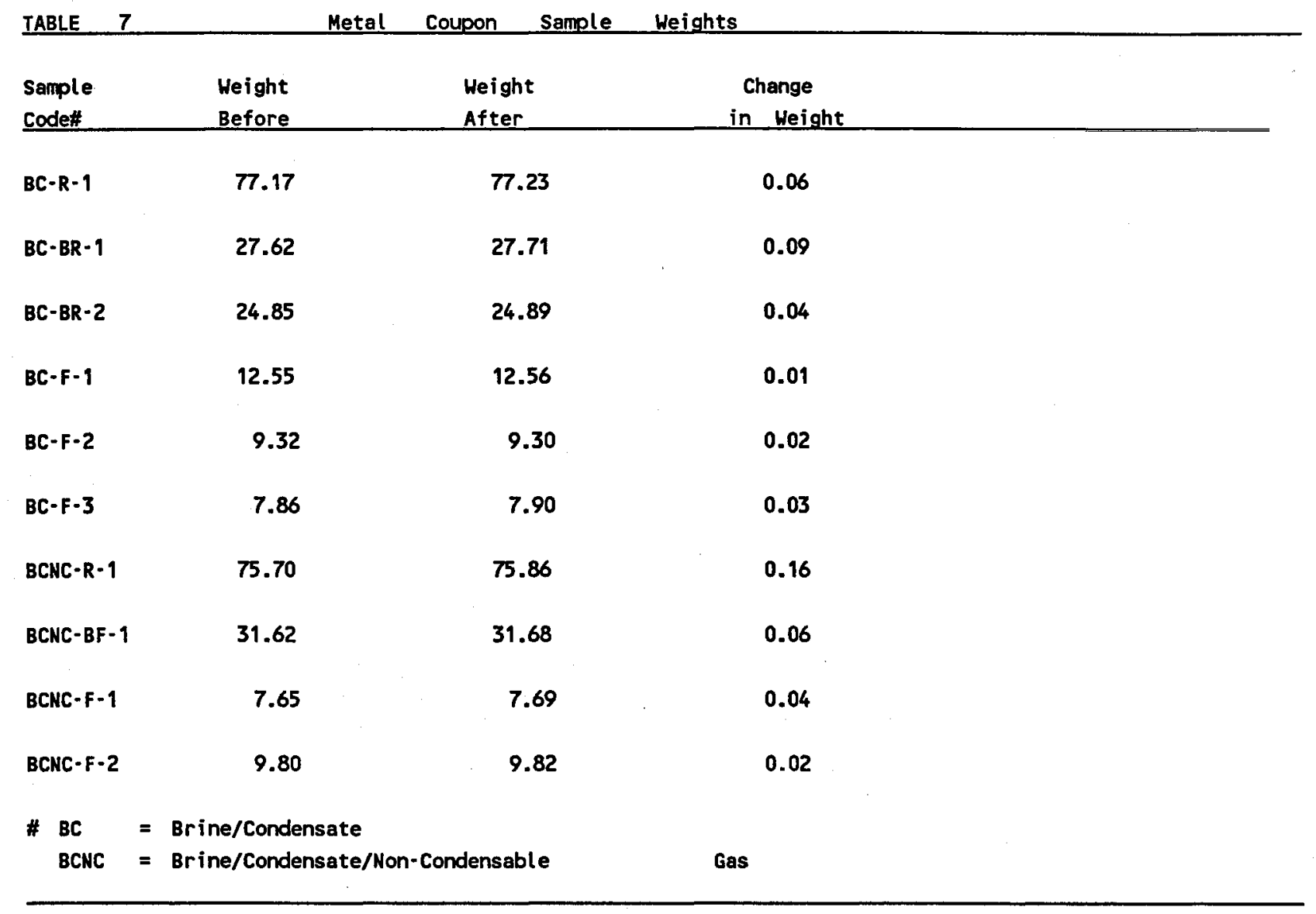

exposure showed very small increases in coupon weights that were only slightly more than the error limits of the balance (Table 7). Scanning electron micrographs of the metal coupons show only minor differences between the exposed and the control samples for both the SS-316L and the SAF-2205 coupons (Plates 4 through 9); the test samples may have trace amounts of solids deposition present but show no indication of pitting or generalized surface corrosion. Examination of several dozen chips each of test and control basalt samples under a light microscope were unable to identify any detectable deposition of silica or sulfides on the exposed chips. 


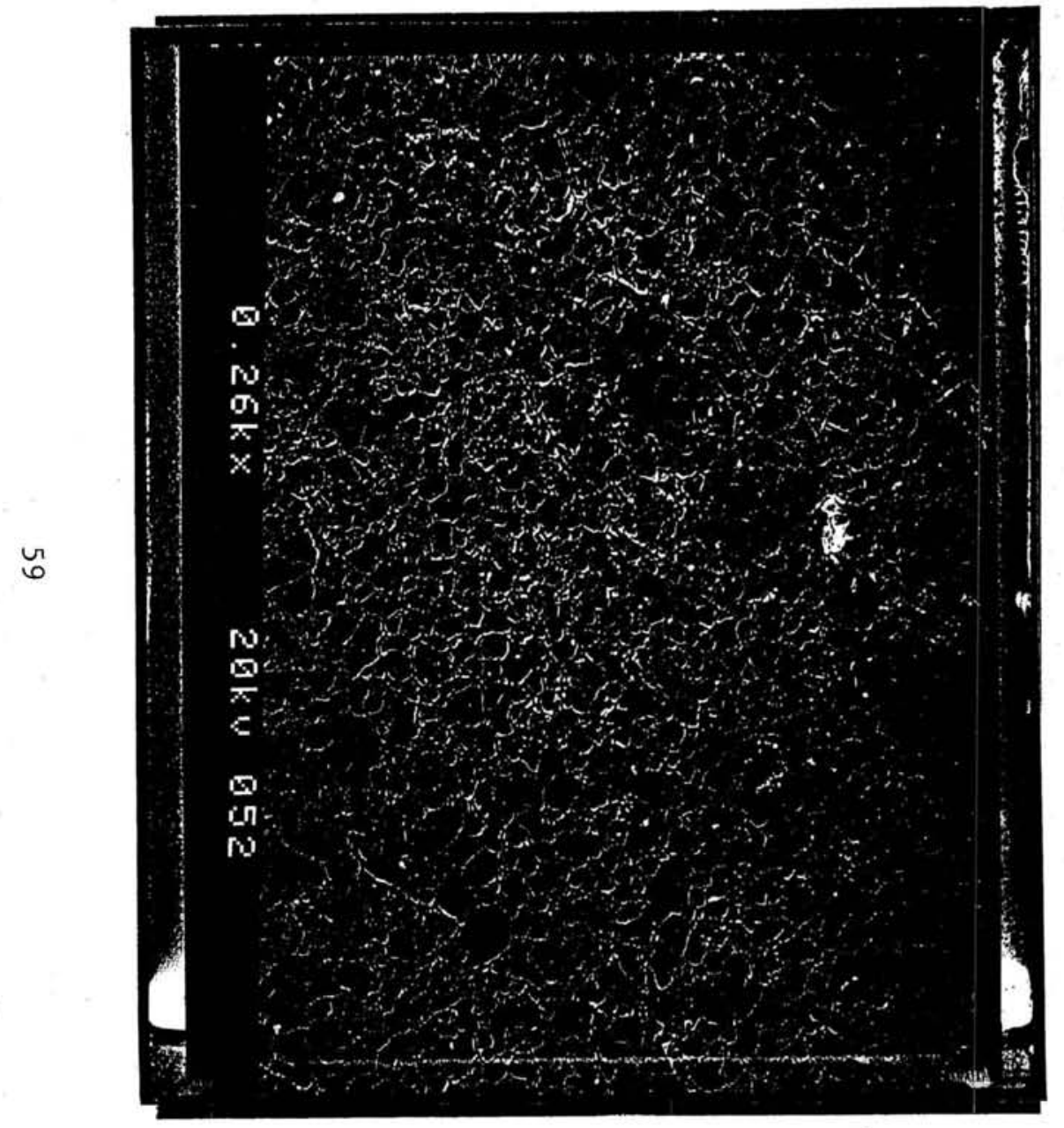

Plate 4. Scanning electron micrograph of SS $316 \mathrm{~L}$ control coupon taken at a magnification of $260 \mathrm{X}$.

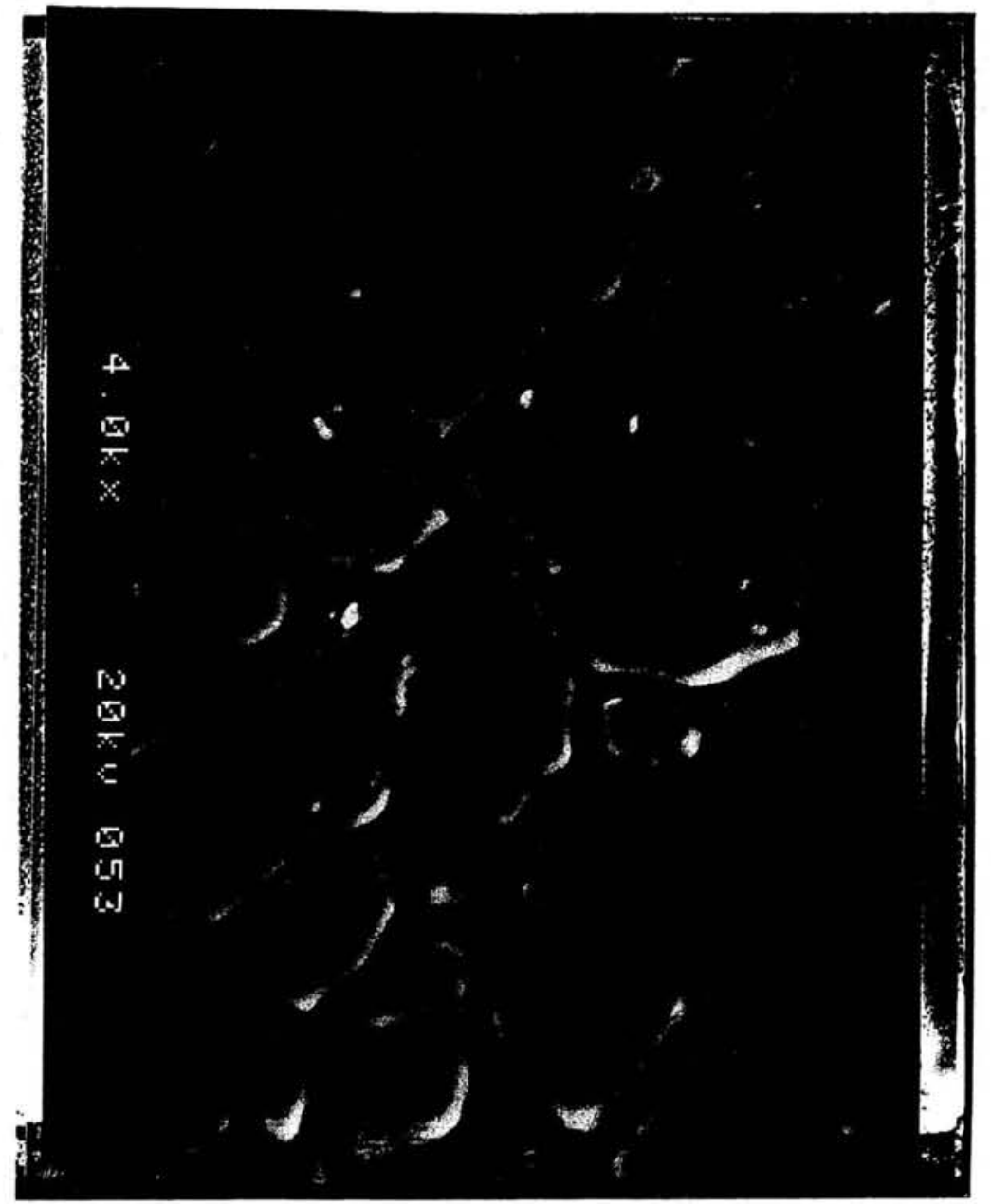

Plate 5. Scanning electron micrograph of SS $316 \mathrm{~L}$ control coupon taken at a magnification of $4000 \mathrm{X}$. 


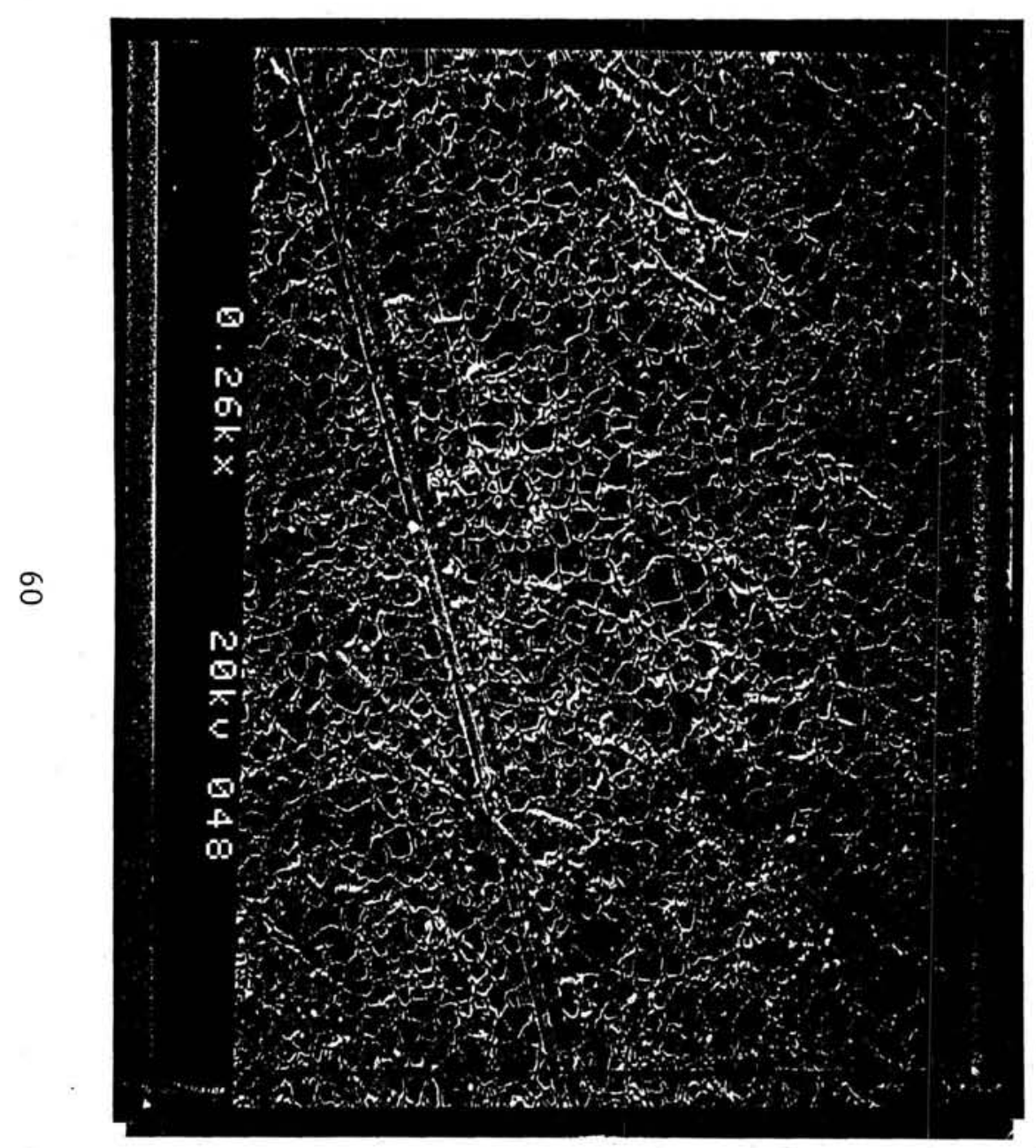

Plate 6. Scanning electron micrograph of SS 316L test coupon after exposure to brine and condensate taken at a magnification of $260 \mathrm{X}$.

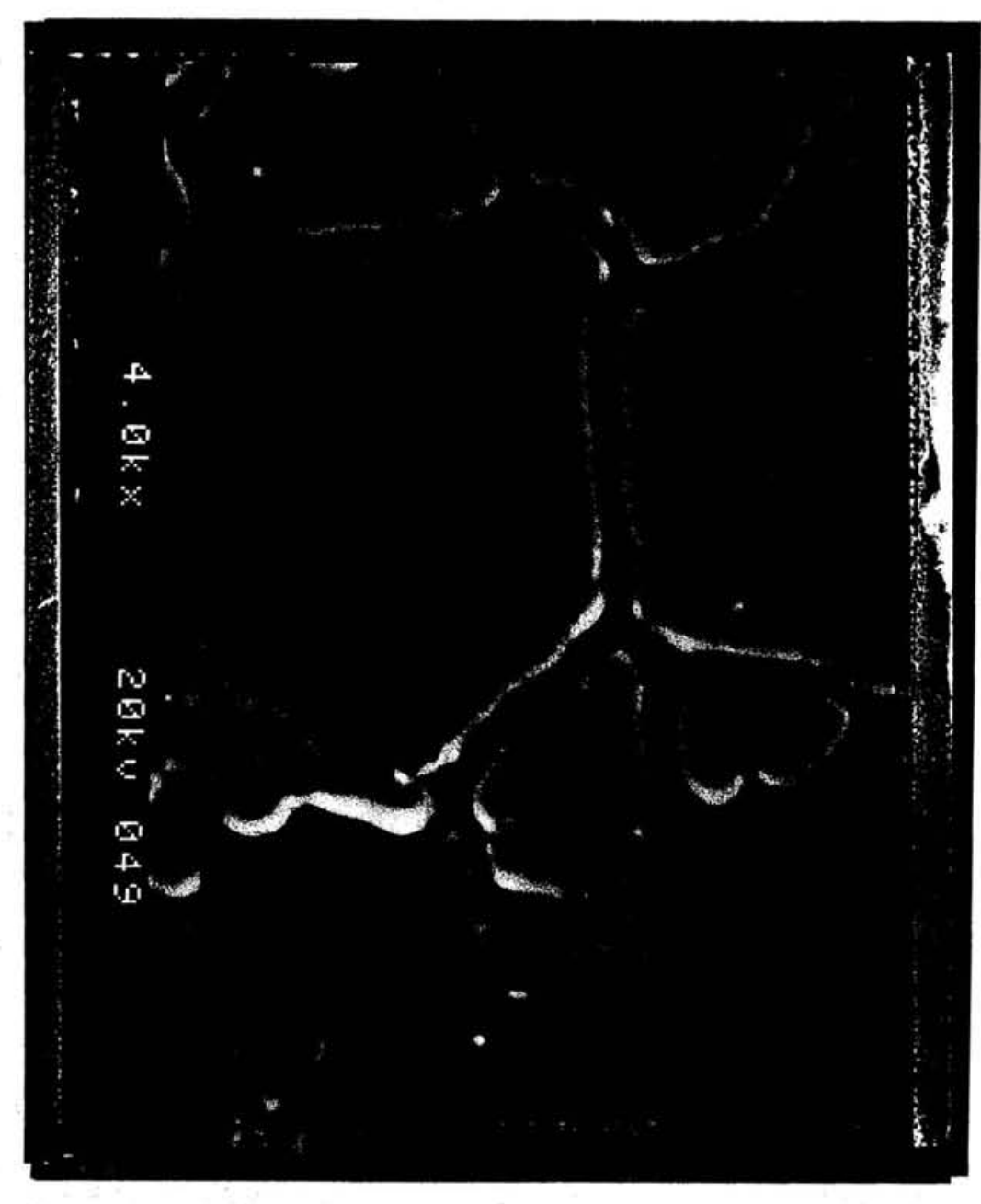

Plate 7. Scanning electron micrograph of SS $316 \mathrm{~L}$ test coupon after exposure to brine and condensate taken at a magnification of $4000 \mathrm{X}$. 


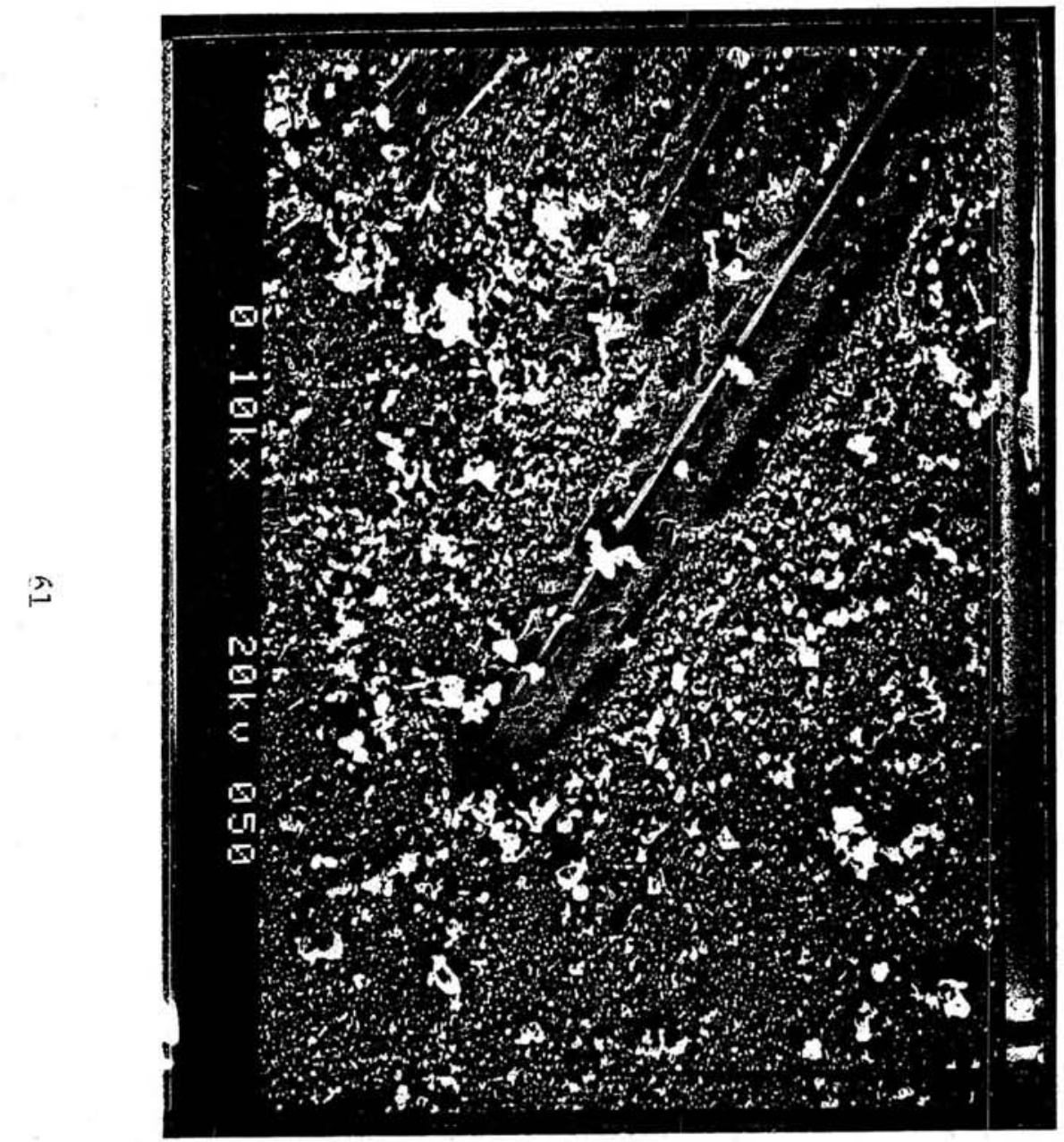

Plate 8. Scanning electron micrograph of SS $316 \mathrm{~L}$ test coupon after exposure to brine, condensate, and non-condensable gas taken at a magnification of $260 \mathrm{X}$.

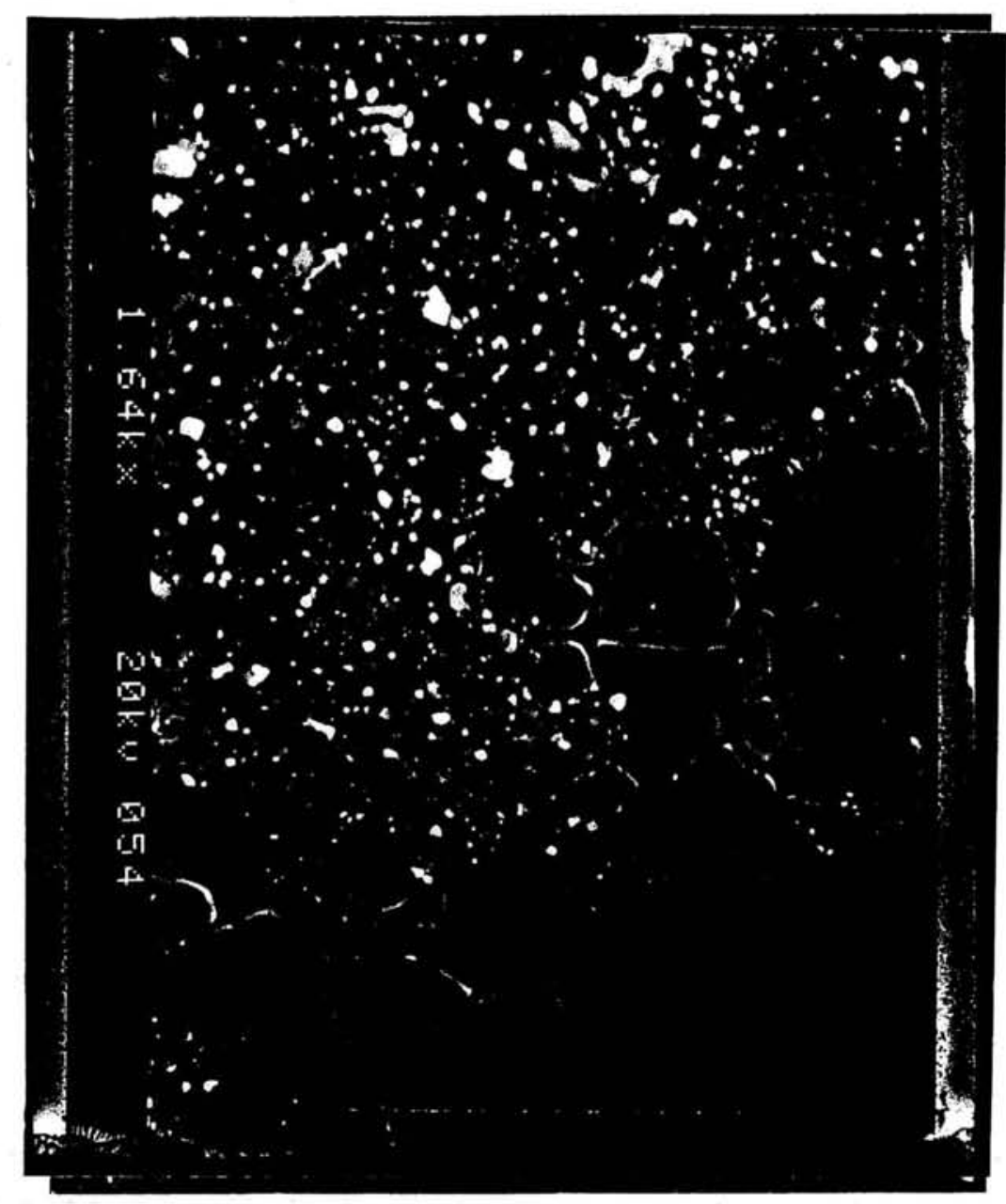

Plate 9. Scanning electron micrograph of SS $316 \mathrm{~L}$ test coupon after exposure to brine, conensate, and non-condensable gas taken at a magnification of $4000 \mathrm{X}$. 


\subsection{BRINE/CONDENSATE/NON-CONDENSABLE GAS TRIAL}

4.6.1 Gas Mixing and $\mathrm{pH}$

sight glass observations revealed that a residual gas phase remained in the flow lines under the experimental temperatures and pressures at all gas injection rates. Extension of the delay line from $4 \mathrm{~m}$ to $35 \mathrm{~m}$ had no detectable effect on the relative proportion of gas to liquid phases even though fluid pH levels showed rapid declines in response to increases in the rate of gas addition. Although it was not possible to sample the residual gases, the high solubility of carbon dioxide (ca. 2500 ppm) and hydrogen sulfide (ca. $3300 \mathrm{ppm}$ ) at the pressures and temperatures of the test suggest that the residual gas phase is predominantly nitrogen and hydrogen which have much lower solubilities (Kohl and Riesenfeld, 1979). At a pressure of 65 bar, however, which would be experienced by the reinjectate at a depth of about $1000 \mathrm{~m}$, even the less soluble nitrogen and hydrogen in the NCG should fully dissolve in the liquid phase.

The non-condensable gases showed a clear influence on the mixed phase $\mathrm{pH}$ levels. Moderate amounts of gas (5 to $10 \mathrm{stp} 1 / \mathrm{m}$ ) injection yielded a drop in $\mathrm{pH}$ from 5.8 to 4.5 . An increase in the gas injection rate to about $30 \mathrm{stp} 1 / \mathrm{m}$ further decreased the fluid $\mathrm{pH}$ to 3.6. Although these values were lower than expected for the weakly acid NCG, after termination of the experiment, it was found that significant amounts of air had been leaking into the noncondensable gas system. Atmospheric oxygen in the NCG oxidized hydrogen sulfide to more acidic sulfur compounds as follows:

$$
\begin{gathered}
\mathrm{H}_{2} \mathrm{~S}+3 \mathrm{O}_{2} \rightarrow \mathrm{SO}_{2}+\mathrm{H}_{2} \mathrm{O} \\
2 \mathrm{H}_{2} \mathrm{~S}+\mathrm{SO}_{2} \rightarrow 3 \mathrm{~S}+2 \mathrm{H}_{2} \mathrm{O} \\
2 \mathrm{SO}_{2}+\mathrm{O}_{2}+2 \mathrm{H}_{2} \mathrm{O} \rightarrow 2 \mathrm{H}_{2} \mathrm{SO}_{4}
\end{gathered}
$$

Hence, the low $\mathrm{pH}$ values are the result of the addition of the more acid, oxidized, sulfur species to the mixed fluids. Exclusion of oxygen from the NCG system, as would occur with a properly operated geothermal facility, would maintain the $\mathrm{pH}$ of the mixed fluids and NCG in the range of 4 to 4.5 , the $\mathrm{pH}$ of the condensate. 


\subsubsection{Homogeneous Precipitation}

Filtration of 1001 of the NCG-treated fluid through a 0.45micron filter yielded only a slight darkening of the filter surface and no detectable increase in back-pressure across the filter. The mass of the solids recovered was about $77 \mathrm{mg}$ for a precipitation rate of $0.769 \mathrm{mg}$ of solid per $\mathrm{kg}$ of fluid. Energy dispersive $\mathrm{x}$-ray and $x$-ray diffraction analyses of the filters showed that the major component of the solids recovered was elemental sulfur with much smaller amounts of iron sulfides and silica. Hence, the major source of particulate in the mixed phase was the result of sulfur production induced by air contamination of the NCG stream.

Twenty-liter filtered fluid samples from this phase of the experiment were permitted to polymerize/precipitate in a closed container for a period of 72 hours. No measurable solids were recovered on filtration after the precipitation period. A suite of smaller volume samples were also held at room temperature, in closed class containers for periods of several weeks after the experiment was concluded. The turbidity of the mixed brine/ condensate remained very low and only gradually began to show evidence of colloidal silica formation by the presence of a faint bluish cast after a period of several months. These samples showed even greater stability than the samples from the prior run for which no NCG had been added to the mixed fluids. Hence, in spite of the limited duration of this experiment, the addition of NCG to a mixed brine/condensate waste stream demonstrated that the reduction in fluid $\mathrm{pH}$ brought about by the injection of the NCG inhibits silica deposition for a period adequate to reinject the waste fluids into the geothermal formation.

\subsubsection{Corrosion and Heterogeneous Precipitation}

The corrosion coupons exposed to the mixed phase fluids showed small, but clearly detectable, amounts of solid deposition on the metal surface. The deposits consisted of fine powdery films that were easily removed by gentle abrasion of the surface. The ease of removal of the scale suggests that the deposits were physically bound to the surface of the metal rather than chemically attached 
to the metal substrate. Scanning electron micrographs of the coupons show that the thickness of the scale layer was less than 10 microns (Plates 8 and 9). Energy dispersive $x$-ray analysis identified sulfur as the predominant constituent with lesser amounts of iron and other transition metal sulfides. The presence of sulfide deposition is consistent with the results of Hirowatari and others (Hirowatari, 1990; Hirowatari and Yamauchi, 1990a, 1990b, 1990c). Silicate deposition was found to be a minor component of the solids deposited on the coupons.

The micrographs also show that metal loss or corrosion resulting from exposure to the fluids was minimal. Beneath the scale layer, the metal surface of the exposed coupons appeared to be nearly identical to the control samples with neither pitting nor generalized corrosion evident. Although the stress corrosion coupons for the SS 316-L and SAF 2205 alloys showed no evidence of cracking or embrittlement in this or the prior trial, a stainless steel sponge used as a solids trap in one of the exposure tanks appeared to suffer extensive embrittlement. Prior to exposure, the metal, which resembled fine lathe turnings, was very flexible; after exposure to the mixed brine/condensate/ $\mathrm{N}-\mathrm{C}$ gases the turnings became brittle and fragile. A similar sample of sponge that was exposed to only the brine/condensate mix in the previous trial did not show an equivalent alteration, suggesting that the high sulfide concentrations in the mixed phase fluids were responsible for the observed changes. The steel alloy from which the sponge was fabricated was type 430 stainless steel.

samples of basalt chips examined under an optical microscope showed deposition of a fine-grained black material on the chip surfaces which was easily washed off. X-ray analysis showed the material to be identical to that present on the corrosion coupons: Predominantly sulfur with smaller amounts of iron sulfides. Amorphous silica was indicated to be a minor constituent of the solids present. 


\subsection{CONCLUSIONS AND RECOMMENDATIONS}

In spite of the relatively short duration of the geothermal fluid recombination experiments conducted at the HGP-A facility, the results show that inhibition of silica precipitation is achievable with the geothermal fluids found in the Kilauea East Rift zone. The addition of steam condensate to the brine phase prevented boiling of the synthetic reinjectate after discharge to atmospheric pressure and inhibited rapid deposition of silica with little sulfide scale formation from the mixed fluids. The injection of non-condensable gases into the reinjectate significantly increased the stability of the dissolved/suspended silica in the fluid but also showed an increase in the rates of sulfur and sulfide deposition. The sulfides were in part the result of $\mathrm{H}_{2} \mathrm{~S}$ reacting with dissolved metal ions in the geothermal fluids, however, some of the iron sulfides, and nearly all of the elemental sulfur, were generated by air entrainment in the NCG system and acid attack of the gas injection system.

The subsidiary tests conducted on alloy compatibilities with the synthetic reinjectate suggest that both SS 316-L and

SAF 2205 are able to tolerate exposure to these fluids whereas SS 430 alloy is subject to sulfide embrittlement when exposed to high partial pressures of hydrogen sulfide. We note, however, that the short duration of these tests may limit the reliability of any conclusions regarding the immunity of the former alloys from attack by the reinjectate. 


\section{SECTION 5}

\section{CONCEPTUAL DESIGN OF A SILICA CONTROL PROCESS}

The NCG/fluid mixing tests demonstrated that silica deposition can, under some conditions, be effectively inhibited in reinjection fluids by the addition of condensate and non-condensable gases to the geothermal waste brines. The basis for control of silica deposition appears to be maintenance of reinjectate $\mathrm{pH}$ in the slightly acid range and possibly the reduction of the degree of silica supersaturation in the liquid phase. Careful application of these control principals to the design of a waste fluid disposal system for commercial scale geothermal power plants may be able to alleviate several of the most intractable operational problems that were encountered at the HGP-A Geothermal Generator Facility.

\subsection{PROCESS DESIGN}

The above silica control principals have been applied to the conceptual design of two fluid disposal systems that we believe could effectively reduce operations and maintenance problems associated with silica deposition, and non-condensable gas disposal, in a commercial geothermal facility in Hawaii. Although the designs are similar in many respects, we believe that it is necessary to treat waste fluids differently according to the levels of sulfide or carbonate reactive metal ions present in the reinjectate fluids. The elements of each system, and the basis for their inclusion in the process design are described below.

\subsection{LOW METALS GEOTHERMAL FLUIDS}

Operating experience at the HGP-A Generator Facility showed that silica deposition can begin immediately after separation of the brine from the geothermal steam phase. Analysis of the silica deposition patterns in the brine handling system showed that deposition occurred downstream of discontinuities and in locations of significant pressure drop in the flow lines. These patterns reflect secondary boiling in the brine line; due to separation of 
the residual NCG into the vapor phase, the brine $\mathrm{pH}$ increases and accelerates silica deposition wherever steam formation has occurred. The presence of steam in the brine piping downstream of the plant separator was confirmed by ultrasonic detection of two phase flow in the discharge system. Because the liquid phase is discharged from the wellhead separator at "saturation" (i.e. the steam vapor pressure at the temperature of separation is equal to the system pressure) steam formation is easily triggered by very small pressure drops within the brine system.

Boiling can be controlled in the brine system by the injection of steam condensate into the flow line immediately downstream of the plant, or wellhead, separator. The volume of water required to eliminate secondary boiling can be calculated on the basis of the saturation temperature of the reinjectate at the minimum system pressure in the reinjection system as follows:

$$
\begin{gathered}
B \times\left(T_{b}\right)+C \times\left(T_{c}\right)=(B+C) \times\left(T_{s}\right) \\
\text { or } \\
C=B \times\left(T_{s}-T_{b}\right) /\left(T_{c}-T_{s}\right)
\end{gathered}
$$

where: $\quad \mathrm{T}_{\mathrm{b}}=$ Brine temperature

$\mathrm{T}_{c}=$ Condensate temperature

$\mathrm{T}_{\mathrm{s}}=$ Saturation temperature at minimum system pressure

For example, given a brine separation temperature of $195^{\circ} \mathrm{C}$, a condensate temperature of $30^{\circ} \mathrm{C}$, and a minimum system pressure of 10 Bars, absolute, the condensate injection rate is as follows:

$$
B \times(195)+C \times(30)=(B+C) \times(181) \text {, }
$$

where $181^{\circ} \mathrm{C}$ is the approximate saturation temperature at $10 \mathrm{Bar}$,

$$
C=0.0927 \times B
$$

Hence, under the above conditions, the addition of $10 \%$ by volume of condensate to the brine flow will prevent steam formation even if the brine pressure falls from its separation pressure of 13.9 Bar to 10 Bar.

The addition of steam condensate to the brine will also serve to decrease the mixed phase $\mathrm{pH}$, due to the NCG present in the 
former, and reduce silica supersaturation by dilution. The combination of these factors should result in a significant decrease in silica deposition rates in the brine handling system.

Two alternate methods of controlling boiling-induced silica deposition were also considered: increase the pressure of the brine in the flow lines by pumping use cold groundwater to decrease the temperature and silica concentration. Pressurization of the brine system has a clear advantage over condensate addition in terms of the capital costs of transmission lines for condensate versus power costs for pumping. However, there is strong potential for cavitation induced boiling upstream of the pump and at its impeller. The cavitation could result in very high rates of silica formation and rapid deterioration of the pumping equipment.

The use of groundwater for cooling the reinjectate may offer some advantages over condensate injection when shallow groundwater is readily available and condensate transmission lines are long. The use of groundwater may, however, require the addition of a weakly acidic buffering agent as well as an oxygen scavenger if $\mathrm{pH}$ and corrosion control are to be maintained. Hence, groundwater may prove to be advantageous under some circumstances, but its chemical composition must be considered in terms of the requirements of the brine phase for controlling silica and corrosion.

The optimal approach toward injection of non-condensable gases into the mixed reinjectate flow will depend on a number of considerations. Potential adverse consequences associated with recombination of the NCG with the brine/condensate flow include: the capital and operating costs of compressing the required gas load; increased frictional losses/pipe diameter for transmission of two-phase flow; an increased potential for the precipitation of insoluble metal sulfides or carbonate scale; and, depending on the $\mathrm{pH}$ of the mixed phase reinjectate, a possible requirement to fabricate the reinjectate line from stainless steel. Although the specific design of a gas injection system must consider each of these potential consequences in terms of specific fluid compositions, several general recommendations can be made. 
The least difficult case is that in which the brine carries a low concentration of sulfide reactive species and the addition of condensate is able to maintain the reinjectate $\mathrm{pH}$ in the range of 5 to 6 . Under these conditions, further addition of NCG to the pressurized brine line would provide little improvement in controlling silica deposition. If, however, there is a drop in the system pressure to below the steam saturation pressure at the reinjection wellhead, secondary steam formation could occur and result in increased silica deposition. Under this circumstance, injection of some NCG at the wellhead may help maintain a low reinjectate $\mathrm{pH}$ in the two-phase interval in the wellbore. The balance of the NCG would then be reinjected at some depth below the water level in the well, and the fluid flow would carry the NCG to the reinjection formation. If saturation pressure is maintained at the wellhead, either because of high back-pressures in the well or intentional addition of condensate, the full gas load could be injected into the liquid flow line immediately downstream of the fluid control valves. This approach would minimize the compressor and pumping costs and would require that only the NCG Iine and the wellbore liner be fabricated of stainless steel.

If additional gas injection is required in order to maintain $\mathrm{pH}$ control in the brine, two approaches could be taken. Additional NCG could be injected directly into the brine system, or the steam condensate could be charged with additional NCG prior to transmission to its injection port on the brine separator. The latter approach offers the advantage of minimizing the capital costs of a gas line and may reduce the power load for compression of the gases. The choice of design options would require that an analysis be performed of the composition of the brine phase and its buffering capacity for the weakly acidic NCG.

\subsection{HIGH METALS GEOTHERMAL FLUIDS}

A more difficult problem is presented with brine compositions that contain significant concentrations of sulfide reactive metals (e.g. iron, copper, lead, zinc, etc.). Under these circumstances; 
addition of sulfide to the brine phase will lead to precipitation of metal sulfides (Hirowatari, 1990, Hirowatari and Yamauchi, 1990a, 1990b) and possibly metal carbonates (Kuwada, 1982), and the generation of a significant suspended solids load in the reinjectate flow. Hence, it would be necessary to install a solids removal system in order to maintain permeability in the reinjection well. Although we did not encounter high particulate sulfide loads in our experimental test apparatus, our results suggest that it is likely that the solids removal system will have to be designed to remove quite small particles from the fluid flow and, hence, may represent a significant capital cost.

If high metal concentrations are present, injection of the minimum NCG volume necessary to reduce these concentrations upstream of the filtration system, followed by injection of the balance at the wellhead, would minimize operating (compressor) costs. Injection of sulfide containing gas into a high metals brine without upstream particulate sulfide removal is considered inadvisable due to the potential for plugging of the reinjection well with sulfides. A gas injection scheme should also consider the potential for formation of insoluble metal carbonates when high concentrations of transition metal and carbonate ions are present in the reinjectate (Kuwada, 1982). Hence, it is recommended that any consideration of gas injection for scale control also include an equilibrium analysis of the mixed phase reinjectate using SolminEq or the WATCH (Kharaka et al., 1988; Svavarsson, 1983) program to determine whether solid precipitates pose a significant risk to reinjection of the mixed fluids.

If it is determined that injection of non-condensable gases will precipitate metal carbonates or sulfides, other commercially available acids or acid buffering agents can be injected into the brine phase and the non-condensable gases be disposed of separately. If this type of silica control process is used, however, one must also consider the potential for the formation of insoluble sulfates (e.g. $\mathrm{CaSO}_{4}, \mathrm{Na}_{2} \mathrm{SO}_{4}$, or $\mathrm{MgSO}_{4}$ ). 


\subsection{SUMMARY}

The addition of a weak acid or an acidic buffering agent to geothermal fluids immediately after separation from the steam phase offers a strong potential for controlling silica precipitation in geothermal brine handling equipment and in reinjection wells. our field experiments indicate that injection of steam condensate and NCG is an effective means of inhibiting silica formation and will enable a geothermal operator to dispose of both liquid and gas phase waste products in an environmentally acceptable manner. The wide recognition that reinjection of fluids back into the formation can be a significant factor in extending the life of the field, and that other methods of treatment of these waste products can involve high capital and chemical costs, the proposed conceptual design may represent both an environmentally and economically beneficial means of disposing of waste fluids from geothermal power generation. 


\section{SECTION 6}

\section{APPLICATION OF SILICA CONTROL TO}

NORTH AMERICAN GEOTHERMAL SYSTEMS

The conceptual design described may be able to alleviate silica scaling in a number of geothermal fields where silica concentrations in waste geothermal brines produce substantial solids deposition problems. In recognition of the limitations posed by the potential for metal sulfide and carbonate deposition, it is acknowledged that not all geothermal fluids carrying high silica loads will be amenable to gas reinjection but will require acidification using industrial acid buffers. Three North American fields for which the treatment system was considered were: Cerro Prieto, Ahuachapan, and Salton Sea. All three have at least some wells that produce brines containing high concentrations of silica, and all have experienced some degree of scaling problems. An analysis of the chemical composition of the fluids at the three fields indicates that gas injection may be appropriate for the first two but not for the last.

\subsection{CERRO PRIETO}

At Cerro Prieto field, silica concentrations in brines discharged from the plant separators range from $830 \mathrm{mg} / \mathrm{kg}$ to $1270 \mathrm{mg} / \mathrm{kg}$ (Hurtado and Murcado, 1990). These fluids have undergone two or three stages of flashing to obtain steam for power generation, and, hence, have lost most of their non-condensable gases. The brines discharged from the power generation process have $\mathrm{pH}$ levels that range from 6.6 up to 8.0 and have TDS concentrations from 17,000 to 42,000. Silica deposition in surface equipment has resulted in significant maintenance costs and has inhibited reinjection of the geothermal waste fluids.

The geothermal brines at cerro Prieto also contain significant concentrations of calcium that, at the high $\mathrm{pH}$ levels of the waste fluids, can undergo ion exchange with dissolved and colloidal silica and substantially accelerate its polymerization and 
coagulation. The pH sensitivity of the ion exchange process suggests that decreasing the $\mathrm{pH}$ of the brines can substantially reduce the rates of silica deposition. Recent testing conducted by Hurtado and Murcado (1990) have demonstrated that reduction of $\mathrm{pH}$ from 8 to 5, through the injection of hydrochloric acid, was able to reduce scaling rates by a factor of twenty.

Injection of NCG into the fluid flow lines after the final stage of brine/steam separation would similarly be expected to reduce silica scaling in the brine piping. The present practice at Cerro Prieto of flashing the separated brines in open silencers and disposing of the waste fluids in open sumps would, however, eliminate most of the advantage gained by injection of gas; flashing in the open silencers would remove most of the injected gas from the fluid, increase the $\mathrm{pH}$, and increase silica deposition in disposal sumps to its present levels. If reinjection of the disposal fluids was undertaken without the final flashing process, injection of gas after the final steam separation could be expected to control silica deposition long enough to reinject the fluids into the deep formation.

Consideration must also be given to the presence of transition metals present in the cerro Prieto fluids. Although one study of the Cerro Prieto fluids indicates iron concentrations of up to 20 $\mathrm{mg} / \mathrm{kg}$ (Mazor and Manon, 1978), another more recent study indicates that some of the wells produce fluids with iron concentrations of $0.3 \mathrm{mg} / \mathrm{l}$ (Truesdell et al., 1981). clearly, the impact of injecting sulfide-rich gases into the iron-rich fluids has a much higher potential for metal sulfide deposition and would probably require separation of metal sulfides from the reinjection fluids. Hence, a detailed feasibility study of this method of silica control will need to consider the metals concentrations in each of the fluid streams selected for treatment.

\subsection{AHUACHAPAN}

Chemical data from the Ahuachapan field are less extensive than those for cerro Prieto, however, reported fluid compositions 
for some of the deep wells indicate silica concentrations of up to $1000 \mathrm{mg} / \mathrm{kg}$ (Campos, 1988). The concentrations of calcium ion in this field are indicated to be a factor of five lower than those found in Cerro Prieto, and the $\mathrm{pH}$ of the brines is neutral to slightly acidic. Hence, the potential for silica scaling in this field is considered to be somewhat lower than that at cerro prieto. None-the-less, if silica concentrations in this range are widely present and are used for power generation, it is likely that silica deposition will become a consideration in the long-term operations of power generation facilities.

If reinjection of fluids is to be pursued in this field, gas injection as a means of $\mathrm{pH}$ control appears to be feasible. Although the data for non-condensable gases in incomplete, the data presented by campos (1988) appears to suggest that hydrogen sulfide concentrations in the geothermal steam may be relatively low. If transition metal concentrations are similar to those of cerro Prieto, it may be possible to inject sufficient gas to control fluid $\mathrm{pH}$ without the need to install a sulfide removal system.

\subsection{SALTON SEA}

The fluids from the salton sea geothermal field have shown a strong tendency to deposit silica-based scale. The presence of extremely high transition metals concentrations - iron in excess of $1000 \mathrm{mg} / \mathrm{kg}$, zinc ranging from 200 to $500 \mathrm{mg} / \mathrm{kg}$, and lead levels of up to $90 \mathrm{mg} / \mathrm{kg}$ (Maimoni, 1982) - indicates that the injection of $\mathrm{CO}_{2}-$ or $\mathrm{H}_{2} \mathrm{~S}-\mathrm{rich}$ gases would be ineffective, if not counter productive, as a means of controlling scale deposition. Studies of the scale deposition occurring at salton sea geothermal facilities, as well as efforts to actively remove base and precious metal ores from the fluids, show that metal sulfide precipitation is a major component of the solids recovered. Hence, reduction of the brine pH may help reduce silica deposition from the salton sea geothermal fluids, but injection of NCG is unlikely to be a successful method of doing so. 


\subsection{SUMMARY AND CONCLUSIONS}

Injection of non-condensable gases into the geothermal fluid streams of operating geothermal facilities may have application to the Cerro Prieto and Ahuachapan geothermal fields if the $\mathrm{pH}$ can be maintained in the slightly acid region throughout the waste fluid disposal system and if the precipitation of sulfide-reactive transition metal ions can be controlled.

Under circumstances when the total dissolved solids concentrations and transition metal ion concentrations are extraordinarily high, as is the case at salton sea, injection of non-condensable gases is unlikely to make a significant contribution to controlling solids deposition from geothermal waste fluids. 


\section{BIBLIOGRAPHY}

Alexander, G.B., W.M. Heston and R.K. Iler, 1954, The solubility of amorphous silica in water, J. Phys. Chem., V. 58, 453-455.

Allen, L.H. and E. Matijevic, 1969, Stability of colloidal silica: I. Effect of simple electrolytes, Jour. Coll. Interf. Sci, v. 31, no. 3, 287-296.

Allen, L.H. and E. Matijevic, 1970, stability of colloidal silica: II. Ion exchange, Jour. Coll. Interf. Sci, v. 33, no. 3, 420-429.

Allen, L.H. and E. Matijevic, 1971, Stability of colloidal silica: II. Effect of hydrolyzable cations, Jour. Coll. Interf. Sci, v. 35, no. $1,66-76$.

Allen, L.H., E. Matijevic, and L. Meites, 1971, Exchange of $\mathrm{Na}^{+}$for the silanolic protons of silica, Inorg. Nucl, Chem. v. 33, 1293-1299.

Baughman, E., R. Uemura, and D.M. Thomas, 1985, Chemistry, scale, and performance of the HGP-A Plant, Electric Power Research Institute Technical Report, $98 \mathrm{pp}$.

Bechtold, M.F., 1955, Polymerization and properties of dilute aqueous silicic acid from cation exchange, J. Phys. Chem., v. 59, 532-541.

Bechtold, M.F., W. Mahler and R.A. Schunn, 1980, Polymerization and polymers of silicic acid, J. of Polymer science: Polymer Chemistry Edition, v. 18, 2823-2855.

Bolt, G.H., 1957, Determination of the charge density of silica sols, J. Phys. Chem., v. 61, 1166-1169.

Campos, T., 1988, Geothermal resources of El Salvador. Preliminary assessment, Geothermics, v. 17, no. 2/3, 319-332.

Carroll, B. and E. Freeman, 1954, The behavior of colloidal silicate solutions as revealed by adsorption indicators, J. Phys. Chem., v. 58, 335-338.

Casey, W.H., A.C. Lasaga and G.V. Gibbs, 1990, Mechanisms of silica dissolution as inferred from the kinetic isotope effect, Geochim. Cosmochim. Acta, v. 54, 3369-3378.

Chemimcal Marketing Reporter, 1992, Chemical commodities prices, Schnell Publishing Co., 27 
Chen, C.A. and W.L. Marshall, 1982, Amorphous silica solubilities IV. Behavior in pure water and aqueous sodium chloride, sodium sulfate, magnesium chloride, and magnesium sulfate solutions up to $350^{\circ} \mathrm{C}$, Geochim. Cosmochim. Acta, v. 46, 279-287.

Crerar, D.A. E.V. Axtmann, and R.C. Axtmann, 1981, Growth and ripening of silica polymers in aqueous solutions, Geochem. Cosmochem. Acta, v. 45, 1259-1266.

De Carlo, E.H. and D.M. Thomas, 1985, Removal of arsenic from geothermal fluids by adsorptive bubble flotation with colloidal ferric hydroxide, Environ. Sci. Tech., v. 19, 538-544.

Decarlo, E.H. and C. Ronay, 1987, Separation of silica from spent geothermal fluids by adsorptive bubble techniques, Sep. Sci. and Tech., Vol 22, no. 4, 1293-1311.

De Jong, B.H.W.S. and G.E. Brown, Jr., 1980, Polymerization of silicate and aluminate tetrahedra in glasses, melts and aqueous solutions--II. The network modifying effects of $\mathrm{Mg}^{2+}$, $\mathrm{K}^{+}, \mathrm{Na}^{+}, \mathrm{Li}^{+}, \mathrm{H}^{+}, \mathrm{OH}^{-}, \mathrm{F}^{-}, \mathrm{Cl}^{+}, \mathrm{H}_{2} \mathrm{O}, \mathrm{CO}_{2}$ and $\mathrm{H}_{3} \mathrm{O}^{+}$on silicate polymers, Geochim. Cosmochim. Acta, v. 44, 1627-1642.

Dove, P.M. and D.A. Crerar, 1990, Kinetics of quartz dissolution in electrolyte solutions using a hydrothermal mixed flow reactor, Geochim. Cosmochim. Acta, v. 54, 955-969.

Fleming, B.A., 1986, Kinetics of reaction between silicic acid and amorphous silica surfaces in $\mathrm{NaCl}$ solutions, J. Colloid and Interface Sci., $v=110$, no. 1, 40-64.

Fleming, B.A. and D.A. Crerar, 1982, Silicic acid ionization and calculation of silica solubility at elevated temperature and $\mathrm{pH}$ : Application to geothermal fluid processing and reinjection, Geothermics, v. 11, no. 1, 15-29.

Fournier, R.O., 1981, Application of water geochemistry to geothermal exploration and reservoir engineering, in: Geothermal systems: Principles and Case Histories, L. Rybach and L.J.P. Muffler eds., John Wiley ad Sons Ltd., New York, 109-143.

Fournier, R.O. and W.L. Marshall, 1983, Calculation of amorphous silica solubilities at $25^{\circ}$ to $300^{\circ} \mathrm{C}$ and apparent cation hydration numbers in aqueous salt solutions using the concept of effective density of water, Geochim. Cosmochim. Acta, v. 47, 587-596. 
Fournier, R.O. and J.J. Rowe, 1966, Estimation of underground temperatures from the silica content of water from hot springs and wet-steam wells, Am. J. Sci., v. 264, 685-697.

Fournier, R.O. and J.J. Rowe, 1977, The solubility of amorphous silica in water at high temperatures and high pressures, Am. Mineral., v. 62, 1052-1056.

Fournier, R.O., and A.H. Truesdell, 1970, Chemical indicators of subsurface temperature applied to hot spring waters of Yellowstone National Park, Wyoming, U.S.A., U.N. Symposium on the Development and Utilization of Geothermal Resources, Pisa, 1970, v. 2, 529-535.

Gallup, D.L., G.R. Anderson, and D. Holligan, 1990, Heavy metal sulfide scaling in a production well at the salton sea geothermal field, Trans. Geoth. Res. Council, v. 14, Part II, 1583-1590.

Goto, K., 1956, Effect of pH on polymerization of silicic acid, $J$. Phys. Chem., v. 60, 1007-1008.

Gregg, S.J. and K.S.W. Sing, 1982, Adsorption surface area and porosity, second Edition, Academic Press, 331 p.

Gudmundsson, J.S., 1983, Silica deposition from geothermal brine at Svartsengi, Iceland, Proc. Inter. Symp. on solving Corrosion \& Scaling Problems in Geothermal Systems, 371-373.

Hauksson, T. and J.S. Gudmundsson, 1986, Silica deposition during injection in svartsengi field, Geothermal Resources Council, v. 10,377 .

Hirowatari, K., 1990, Experimental study on scale prevention method using exhausted gases from geothermal power station, Nippon Chinetsu Gakkaishi (Japan), v. 12, no. 4, 347-362.

Hirowatari, K. and M. Yamauchi, 1990a, Scale preventing method for a geothermal power generation device, Japan Patent 2-256882.

Hirowatari, K. and M. Yamauchi, 1990b, Development of technology to prevent scale from adhering, with use of exhaust gas from geothermal power station, Kenkyu Kiho (Kyushu Denryoku) (Japan), no. 71, 161-189.

Hirowatari, K. and M. Yamauchi, 1990C, Experimental study of scale prevention using exhausted gases from geothermal power station, Geothermal Resources Council 1990 International Symposium on Geothermal Energy Transactions, Part II, 1599-1602. 
Horne, R.N., 1981, Geothermal reinjection experience in Japan, Society of Petroleum Engineers.

Iglesias, E.R. and O. Weres, 1981, Theoretical studies of Cerro Prieto brines' chemical equilibria, Geothermics, v. 10, no. $3 / 4,239-244$.

Iler, R.K., 1975, Coagulation of colloidal silica by calcium ions, mechanism, and effect of particle size, Jour. Coll. Interf. Sci., v. 53, no. 3, 476-488.

Iler, R.K., 1979, The chemistry of silica: solubility, polymerization, colloid and surface properties, and biochemistry, John Wiley and Sons, New York, $866 \mathrm{pp}$.

Kharaka, Y.K., W.D. Gunter, P.K. Aggarwal, E.H. Perkins, and J.D. DeBraal, 1988, SolminEq.88: A computer program for geochemical modeling of water-rock interactions, U.S. Geological Survey Water Res. Invest. Rep. 88-4227, Menlo Park, CA, 165 p.

Kohl, A.L. and F.C. Riesenfeld, 1979, Gas Purification, Gulf Publishing Co., Houston, Tx. 825 pp.

Kroopnick, P.M., D.M. Thomas, L.S. Lau, R.W. Buddemeier, and D. Bills, 1980, Geochemical Techniques in geothermal research The Hawaii example, in J.M. Tater (ed.), Alpine-Himalayan Region, Tectonophysics, v. 62, 87-97.

Kuwada, J., 1982, Field demonstration ofthe EFP system for carbonate scale control, Geoth. Res. Counc. Bull., v. 11, no. $9,3-9$.

Lazarev, V.B., G.P. Panasyuk, G.P. Boudova and I.L. Voroshilov, 1982, Thermogravimetric study of the kinetics of structural relaxation of amorphous silica, J. of Thermal Analysis, v. 23, 73-81.

Makrides, A.C., M. Turner, and J. Slaughter, 1979, Condensation of silicic acid solutions, Jour, coll. Interf. Sci., v. 73, no. $2,345-367$.

Marshall, W.L., 1980, Amorphous silica solubilities -- III. Activity coefficient relations and predictions of solubility behavior in salt solutions, $0-350^{\circ} \mathrm{C}$, Geochim. Cosmochim. Acta, v. $44,925-931$.

Marshall, W.L. and C.A. Chen, 1982a, Amorphous silica solubilities - $v$. Predictions of solubility behavior in aqueous mixed electrolyte solutions to $300^{\circ} \mathrm{C}$, Geochim. Cosmochim. Acta, v. $46,289-291$. 
Marshall, W.L. and C.A. Chen, 1982b, Amorphous silica solubilities -- VI. Postulated sulfate-silicic acid solution complex, Geochim. Cosmochim. Acta, v. 46, 367-370.

Marshall, W.L. and J.M. Warakomski, 1980, Amorphous silica solubilities -- II. Effect of aqueous salt solutions at $25^{\circ} \mathrm{C}$, Geochim. Cosmochim. Acta, v. 44, 915-924.

Mazor, E. and A. Manon M., 1978, Geochemical tracing in producing geothermal fields: A case study at cerro Prieto, First Symposium on the cerro Prieto Geothermal Field, Baja California, Mexico. Earth Sci. Div. Lawrence Berkeley Laboratory, CA, 102-112.

Morey, G.W., R.O. Fournier, and J.J. Rowe, 1964, The solubility of amorphous silica at $25^{\circ} \mathrm{C}$, Jour. Geophys. Res., v. 69, no. 10, 1995-2002

Okamoto, G., T. Okura and K. Goto, 1957, Properties of silica in water, Geochim. Cosmochim. Acta, v. 12, 123-132.

Ontiveros, C., 1988, Polymerization and deposition of silica in aqueous solutions, UMI Dissertation Information Service.

Parks, G.A., 1965, The isoelectric points of solid oxides, solid hydroxides, and aqueous hydroxo complex systems, Chem. Rev., v. $65,177-198$.

Rimstidt, J.D. and H.L. Barnes, 1980, The kinetics of silica-water reactions, Geochim. Cosmochim. Acta, v. 44, 1683-1699.

Rothbaum, H.P., B.H. Anderton, R.F. Harrison, A.G. Rohde, and A. Slatter, 1979, Effect of silica polymerisation and $\mathrm{pH}$ on geothermal scaling, Geothermics, v. 8, 1-20.

Shannon, W.T., W.R. Owers and H.P. Rothbaum, 1982, Pilot scale solids/liquid separation in hot geothermal discharge waters using dissolved air flotation, Geothermics, v. 11, 43-58.

Strickland, J.D.H. and T.R. Parsons, 1972, Determination of reactive silicate. In: A practical Handbook of Seawater Analysis, 65-70. Fisheries Research Board of Canada.

Svavarsson, H., 1983, The WATCHI and the WATCH3 programs - vehicles for interpreting chemical analyses of geothermal water. $A$ guide for users. Orkustofnun, Geothermal Division, Reykjavik, Iceland, $38 \mathrm{p}$.

Truesdell, A.H., J.M. Thompson, T.B. Coplen, N.L. Nehring and C.J. Janik, 1981, The origin of the cerro Prieto geothermal brine, Geothermics, v. 10 , no. 3/4, 225-238. 
Weres, O. and L. Tsao, 1981, Chemistry of silica in Cerro Prieto brines, Geothermics, v. 10, no. 3/4, 255-276.

Weres, O., A. Yee and L. Tsao, 1980, Kinetics of silica polymerization, Lawrence Berkeley Laboratory, LBL-7033 UC-4, Berkeley, CA, $256 \mathrm{p}$.

White, D.E., W.W. Brannock and K.J. Murata, 1956, Silica in hotspring waters, Geochim. Cosmochim. Acta, v. 10, 27-59.

Wirth, G.S. and J.M. Gieskes, 1978, The initial kinetics of the dissolution of vitreous silica in aqueous media, J. Colloid and Interface Sci., v. 68, no. 3, 492-500.

Yokoyama, T., Y. Takahashi, C. Yamanaka and T. Tarutani, 1989, Effect of aluminum on the polymerization of silicic acid in aqueous solutions and the deposition of silica, Geothermics, v. 18 , no. $1 / 2,321-325$. 


\section{APPENDIX I \\ STATEMENT OF WORK}

The tasks that are to be undertaken under the present study are as follows:

4.1 Polymerization studies. Complete an analysis of the rate of polymerization of the dissolved silica from the geothermal brine at temperatures ranging from 100 degrees $C$ down to 30 degrees $c$, and after the addition of the following reagents: acid and caustic; iron sulfate; and potassium aluminum sulfate. Investigate other commercially available reagents for scale/polymerization control to determine whether it will be possible to either better accelerate or to retard the rate of silica polymerization.

4.2 Low-temperature Brine Treatment. Conduct an experiment to treat a continuous side stream of brine (cooled to temperatures of less than 100 degrees $C$ ) with $\mathrm{pH}$ control and metal ion reagents at the optimum levels determined in the polymerization studies. Analyze the depositional characteristics of the brine for settling efficiency, recovery rates, and fouling temperatures of less than 100 degrees $C$. Analyze the effect of reagent addition and retention times on the rate of silica deposition inside the treatment system, in the heat exchanger, and in a retention volume at the outlet of the treatment system. Test the efficiency of removal of the silica from the low temperature fluids. Design a hightemperature pressurized treatment system.

4.3 Fabricate a pilot scale treatment system based on the results of subtask 4.2. Conduct tests with pH control and reagent addition to determine the effects of the brine treatment on the deposition rate of silica in the piping system and in the heat exchanger.

4.4 Fluid Characterization. Analyze the discharge fluids from subtask 4.3 for solids settling rates and residual silica concentrations. Conduct a particle size/fouling experiment to determine fouling rates of filters having varying pore sizes.

4.5 Preliminary Design of Pilot scale system. Prepare a preliminary design for a larger scale silica treatment system capable of handling the full brine load from the HGP-A geothermal generator.

4.6 Byproduct Characterization. Retain the precipitated silica recovered from the long-term operation of the small scale treatment system and analyze this silica for its physical and chemical characteristics that are relevant to possible commercial use. Analyze for particle sizes, specific surface 
areas, overall purity, and concentration of key coloration elements such as iron, zinc, and manganese. Investigate potential uses for the silica and determine the silica removal and treatment conditions that will optimize the most valuable characteristics of the recovered byproduct.

4.7 Preliminary Evaluation of the Applicability of Test Results to other Geothermal Fluids. In collaboration with researchers and companies at other North American geothermal fields, conduct a preliminary assessment of the applicability of the treatment techniques developed under task 4.1 through 4.5 to silica-laden geothermal fluids from North American systems. This task shall include contacting companies in the Imperial Valley or Cerro Prieto to obtain fluid chemistry and process chemistry currently being applied. It will then be determined if the treatment methods developed in Task 4.1 through 4.5 might be successfully applied to these geothermal systems. 


\section{APPENDIX II}

\section{EXPERIMENTAL PROTOCOL FOR GAS INJECTION TEST SYSTEM}

Two experimental runs were conducted: The first evaluated the impacts of mixing the brine and steam condensate streams, and the secona examined the response of the fluids to mixing all three streams, brine, condensate, and non-condensable gases, together.

Preparation of the system for a brine/condensate mixing run included the following procedures:

The exposure tanks used for heterogeneous precipitation of solids from the fluid were loaded: one with fine basalt chips and the other with pre-weighed metal coupons. The basalt chips were sized to dimensions of $3 \mathrm{~mm}$ or less and were washed both prior to and after loading into the exposure tank. The metal coupons used for the exposure experiments were supplied by Puna Geothermal Venture, and were fabricated from SAF 2205 and SS 316L alloys. Two coupons of each alloy were used. One coupon was installed as received and one was clamped in a "U" configuration to test for stress corrosion. The exposure tanks were installed on the system in a parallel configuration to pass mixed fluid through both tanks for the duration of the mixing run.

The system was pressure tested using steam condensate to confirm that any leaks within the system were of a manageable size. (Because the system was fabricated of threaded stainless steel components, a completely leak proof system was not considered to be reasonably achievable.)

start-up of the system was initiated by pressurizing the flow line and establishing fluid flow through the pumped condensate injection system. Condensate flow was initially set at 11 l/min, at a pressure of 15 bar, through manipulation of a pump by-pass system, the inlet valves, and the discharge valve manifold. After temperature and flow equilibrium were achieved with condensate alone, brine was admitted into the system, and the flow of each constituent was balanced to provide the appropriate temperature and silica concentration in the mixed phase. Although the experimental 
plan was to determine the brine flow by difference between the measured condensate and mixed flow-sensors, the mixed-flow sensor failed to operate properly. An alternate method was used to determine brine flow that relied on the heat balance among the brine, condensate, and mixed phases. Because heat is a conservative parameter under the experimental conditions, an enthalpy balance calculation was a more certain measure of the proportions of brine and condensate because mechanical errors were not a factor in the calculations. Care was required in establishing brine flow into the system in order to balance the flow rate and pressure at a point where the brine could be admitted to the system without allowing it to flash to steam. Prevention of brine flashing was considered critical to both the integrity of the system -- due to hammering of the steam as it was condensed in the mixed phase line -- as well as in the prevention of scale nucleation that is triggered by the boiling process.

During the initial start-up, we found that both the temperature and fluid flow rates showed a cyclic behavior that was induced by operation of the plant separator level control valve. Because it was not possible to modify plant operations without risking serious consequences, the inlet and discharge systems were set to minimize the flow rate variations and the testing was continued with temperature excursions spanning a range from $91 .{ }^{\circ} \mathrm{C}$ $\left(190^{\circ} \mathrm{F}\right)$ to $105^{\circ} \mathrm{C}\left(220^{\circ} \mathrm{F}\right)$. The brine and condensate flow rate, and the silica and chloride concentrations, that correspond to these temperatures are presented in Table 1.

The continuous flow test was started, and after establishing as stable a flow as possible at about $11 \mathrm{lpm}$ and $100^{\circ} \mathrm{C}$, flow through the system was partially diverted to a bypass line that permitted observation of the.fluid through the sight glass to determine whether significant precipitation was occurring. Further evaluation of precipitation rates were also undertaken by installing a pressurized filter system on a side stream of the mixed phase flow. The filters employed were $90 \mathrm{~mm}$ in diameter and 
0.45 micron pore size and passed up to 2001 of mixed fluid to determine the mass of precipitate present in the volume of fluid passed. The unfiltered stream was split and passed through two parallel exposure tanks to evaluate solids deposition onto both metal substrates and onto basalt chips. The fluid from the exposure tanks was then discharged through the fluid disposal system into the brine sump.

Continuous flow through the system was maintained for eight days. There were, however, two periods during which fluid flow conditions were modified substantially due to loss of the condensate pump electrical supply. During the interruptions the system was exposed only condensate at a reduced pressure and flow rate for periods of 3 hours and 5 hours, respectively, during which time repairs were made to the pump breaker and the system brought back up to operating pressure and stable flow.

In anticipation of the imminent shutdown of the HGP-A facility, it was decided that adequate data had been collected after eight days of brine/condensate mixed flow so this phase of the experiment was terminated. Immediately after completion of the test run, the corrosion coupons were retrieved and samples of the basalt chips were recovered from the exposure tanks. The corrosion coupons were reweighed and stored in plastic bags; the basalt chips were saved for microscopic examination.

Prior to the initiation of the second experiment, the sample exposure tanks were washed with fresh water and re-loaded with weighed stainless steel coupons and with a fresh charge of basalt chips. Condensate and brine flow through the test system were reestablished as described for the first experimental run and after flow stabilization was achieved, gas injection into the liquid flow stream was initiated. The system was operated for 36 hours before it became necessary to suspend operations in order to replace a leaking valve and extend the delay coil on the test system. The system was operated in this configuration for a period of 48 hours before failure of the gas metering device forced us to halt the gas injection phase of the experiment. Because the HGP-A Generator 
Facility was required to terminate its operations forty-eight hours later, it was not possible to repair the gas metering device and continue operations. At the conclusion of the gas injection test, the metal coupons and samples of the basalt chips were recovered from the exposure tanks. Over the course of this run samples of filtered fluid were also recovered following an identical protocol to that used in the fluid mixing experiment.

A list of the equipment used and the suppliers from whom the equipment was purchased is found in Table Al. 


\begin{tabular}{|c|c|}
\hline $\mathrm{N}-\mathrm{C}$ Gas Compressor & $\begin{array}{l}\text { Corken } \\
\text { Model D191AM9FDBA }\end{array}$ \\
\hline Condensate Pump & $\begin{array}{l}\text { Oberdorfer } \\
\text { Model } 9156 \mathrm{C}\end{array}$ \\
\hline $\begin{array}{l}\text { Sensing Equipment } \\
\text { pH Meter } \\
\text { pH Probe } \\
\text { High Temp. Liquid Flow Meter } \\
\text { Low Temp. Liquid Flow Meter } \\
\text { Gas Flow Meter }\end{array}$ & $\begin{array}{l}\text { Omega Engineering } \\
\text { PHTX-92 } \\
\text { PHE } 5431-10 \\
\text { FP-5210 } \\
\text { FP-5300 } \\
\text { FMA }-5700\end{array}$ \\
\hline Particle Filter & $\begin{array}{l}\text { Cole Parmer Inst. Supply } \\
\text { Model N-02927-50 }\end{array}$ \\
\hline Caustic Metering Pump & $\begin{array}{l}\text { Ryan Herco } \\
\text { Model A141-155 }\end{array}$ \\
\hline Cooling water Pump & $\begin{array}{l}\text { Little Giant } \\
\text { TE-7-MD-HC }\end{array}$ \\
\hline Pressure Transducers & $\begin{array}{l}\text { National Semiconductor } \\
\text { Model LX1430 }\end{array}$ \\
\hline Valves and Fittings & Swagelok \\
\hline
\end{tabular}

